\title{
A Search for the Electric Dipole of the Electron
}

\author{
Kamal Fuad Abdullah \\ (Ph.D. Thesis) \\ Physics Department \\ Univeristy of California
}

and
Materials and Chemical Sciences Division
Lawrence Berkeley Laboratory
1 Cyclotron Road
Berkeley, California 94720
USA

August 1989

Supported through the Lawrence Berkeley Laboratory's Experimental Research and Development Fund Program, by the U.S. Department of Energy under Conuract Number DE-AC03-76SF00098. 
A Search for the Electric Dipole Moment of the Electron

by

Kamal Abdullah

\begin{abstract}
We report a new upper limit on the electric dipole moment (EDM) of the electron of $\mathrm{d}_{e}=0.1 \pm 3.2 \times 10^{-26} \mathrm{e}-\mathrm{cm}$. This precision is one hundred times better than any previously published limit and a factor of two better than that of unofficial reports.

Recently there has been a great deal of theoretical interest in the possibility of a nonzero electron EDM. Models such as the left-right-symmetric Standard Model and an "offstandard" model with new heavy neutrinos are constrained by the new limit on $\mathrm{d}_{\mathrm{e}}$.

A non-zero electron EDM would violate the time reversal $(T)$ and parity $(P)$ spacetime symmetries. T-violation was observed in neutral kaon decay and is still not fully explained by the Standard Model.

Our experimental technique involves searching for an energy shift, linear in applied electric field, between the $m_{F}=1$ and $m_{F}=-1$ magnetic sublevels of the $F=1$ hyperfine level of the $6^{2} p_{1 / 2}$ ground state of atomic thallium. If the electron has a non-zero EDM, this thallium state will exhibit an atomic electric dipole moment that is roughly 600 times larger.
\end{abstract}

The energy shift is detected with the technique of magnetic resonance spectroscopy, employing separated oscillating fields, applied to an atomic beam of thallium. In the 
approach, any relative phase-shift between the $m_{F}= \pm 1$ components of the $F=1$ wavefunction acquired by the atom as it travels through an electric field is detected through interference with two separate oscillating magnetic fields located on either side of the electric field.

The new level of precision is achieved through several improvements on previous experiments including employment of a vertical apparatus, two opposing atomic beams, and optical pumping for atomic state selection and analysis.

Noise and systematics are greatly suppressed by chopping the direction of the electric field, the direction of the atomic beam, and the relative phase between the two oscillating magnetic fields.

This measurement is the first step in an experiment in which it is hoped to measure $d_{e}$ to a precision of $10^{-27} \mathrm{e}-\mathrm{cm}$ or better. 


\section{Table of Contents}

1. Introduction.....................................................

A. Why Measure the EDM of the Electron?....................................... 1

B. History and Limits on the Electron EDM......................................2

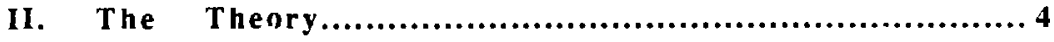

A. The CP Violation Puzzle......................................................4

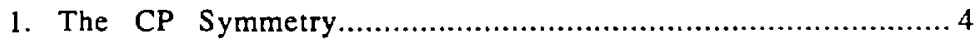

2. Baryon Asymmetry in the Universe........................................... 4

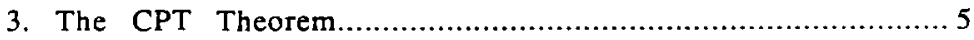

4. CP Violation in the Standard Model......................................... 6

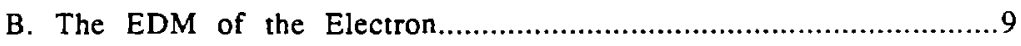

1. A Nonzero Electron EDM Violates CP Invariance............................. 9

2. Predicted Electron EDM's for a Variety of Field Theories.....................10

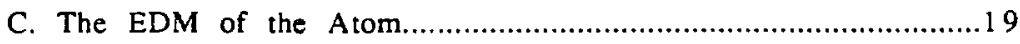

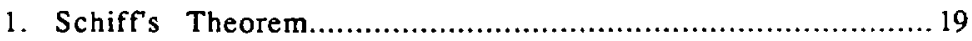

2. Calculating the Enhancement Factor..........................................21

III. The Experiment..........................................26

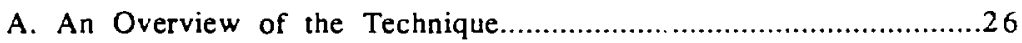

B. The Atomic Beam....................................................... 31

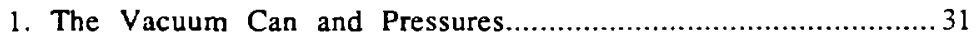

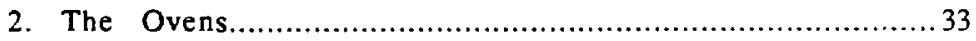

3. The Atomic Flux and Velocity Distribution....................................35

C. Polarizing and Analyzing with Optical Pumping................................40

1. The Optical Pumping Technique.........................................40 
2. Rate Equations and Optical Linewidth........................................43

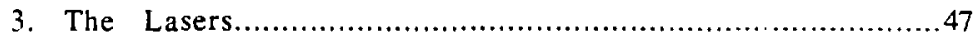

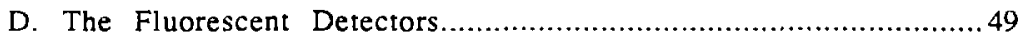

E. The Electric Field.......................................................... 52

1. The Electric Field Plates......................................................52

2. The High Voltage Switch and Charging Time.............................. 54

F. The Magnetic Field................................................... 55

1. A Schematic of the Coils and Shields...........................................55

2. The Coils and Field Homogeneity ..........................................56

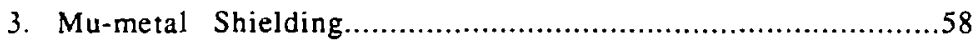

G. Radiofrequency Transitions.................................................61

1. The "Hairpin" Loops......................................................61

2. The RF Synthesizer and Phase-Shifter Circuit................................62

3. Radiofrequency Transitions in the RF Loops.................................63

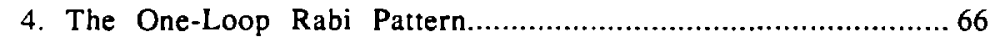

H. Two-Loop Signals - the Evolution of an Atom's Wavefunction..................69

1. The Signal for Arbitrary RF Phase, Power, and Frequency.................. 69

2. The Signal Near Resonance - the Central Fringe Approximation...........71

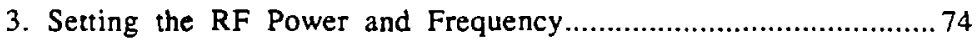

I. Extracting the EDM from the Raw Signal....................................... 75

1. The Form and Size of the EDM, the vxE, Magnetic Noise Phase-Shifts.... 75

2. The RF Phase Chop, the E-Field Chop, and the Atomic Beam Chop........77

3. Analyzing Powers and their Calibration...................................... 80

J. Systematics...................................................... 82

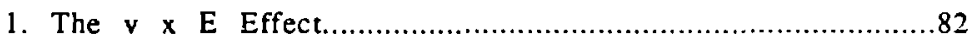

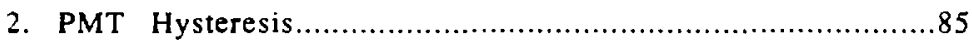

3. Numerical Simulation of Possible Systematic Sources........................85 


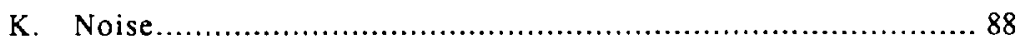

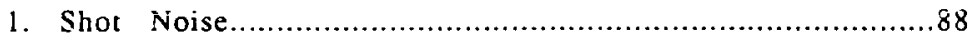

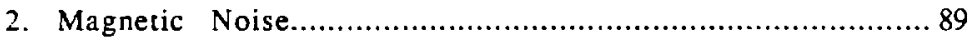

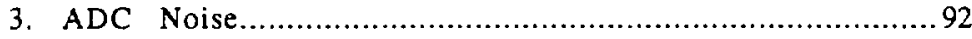

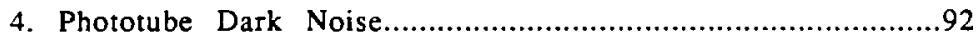

5. Scattered A:oms and Laser Power Fluctuations...............................92

6. Atomic Beam Fluctuations...............................................93

IV. Results and Conclusions....................................94

A. The Data Collection Cycle................................................... 94

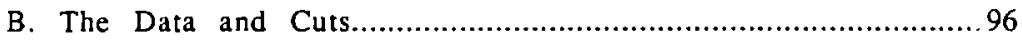

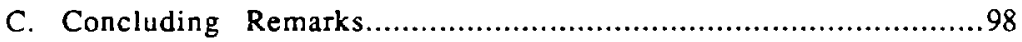

Appendix A - CP Violation in the Dirac Lagrangian for a Fermion with an EDM.... 100 Appendix B - A Calculation of the EDM in a Theory with Horizontal Couplings.. 102 Appendix C - A Model Ramsey Magnetic Resonance Experiment..................... 105 Appendix D- The Low Lying Energy Levels of Thallium................................107 Appendix E- The Lande $g$-factor of the $\mathrm{TT}^{205} 6^{2} \mathrm{P}_{1 / 2}(\mathrm{~F}=1)$ State ....................108 Appendix F- The Computer and Data Collection Electronics.......................... 109 Appendix G- A Clossary of Symbols and Constants...................................111 


\section{Introduction}

\section{A. Why Measure the Electron EDM?}

There is strong reason to believe that an improved measurement of the electron electric dipole moment (EDM) is both timely and useful in shaping today's view of particle physics. A non-vanishing EDM in an elementary particle would violate $\mathrm{CP}$ invariance, the product of charge conjugation $(\mathrm{C})$ and parity $(\mathrm{P})$. Although $\mathrm{CP}$ violation was discovered in a single system in the 1960 's, it still remains one of the most challenging problems in physics.

In addition, there has recently been a great deal of theoretical interest in models that suggest or allow large, measurable electron EDM's. These new theories range from simple extensions of the standard $S U(3)_{C} \otimes S U(2)_{L} \otimes U(1)_{Y}$ model to the more revolutionary such as supersurings and supersymmetry. It is interesting to note that proving or disproving most of these models is beyond the capabilities of any foreseeable particle accelerator. However, careful measurements of the EDM's of fundamental particles provide valuable, albeit limited, insights into these new theories.

It would be incorrect to view measuring the electron EDM as probing the internal structure of an electron. Consider the anomalous $g$ value $g \neq 2$ of the electon. Its value is not determined by anything internal to the electron. In the framework of modern physics, the anomalous magnetic moment arises from the electron's interaction with the gauge field in quantum electrodynamics. Here the gauge field is the photon field. Similarly, the electric dipole moment would arise from interactions with more complicated gauge fields (those that incorporate $\mathrm{CP}$ violation). Thus, by studying the electron EDM, we are not just examining a single system, but fundamental interactions in general. 


\section{B. History and Limits on the Electron EDM}

Soon after the first experiments involving the formation and decay of positronium in an electric field, there was renewed interest in the question of an intrinsic electron EDM. After Lee and Yang ${ }^{1}$ first suggested that parity might be violated in weak interactions, Landau 2 showed that the existence of an intrinsic EDM in an elementary particle would violate $\mathrm{CP}$. Thus began the search for the electric dipole moment of the electron.

In 1958, E. Salpeter ${ }^{3}$ examined previous atomic experiments and deduced an upper limit on the electron EDM. In particular, he looked at the Lamb shift and the lifetime of the metastable 2 s-state of hydrogen. He deduced a limit of roughly $d_{e}<10^{-13} \mathrm{e}-\mathrm{cm}$, where $d_{e}$ is the electron EDM and $e$ is the charge of the electron.

The prospects of finding a non-zero EDM in either a nucleus or atom must have seemed quite grim when Schiff $f^{4}$ introduced his famous theorem in 1963. His theorem states that any non-relativistic, quanrum mechanical system consisting of point, charged, electric dipoles has a vanishing electric dipole moment to second order.

However, P.G.H. Sandars 5 soon demonstrated that Schiffs theorem fails when relativistic effects are included. In fact, he found that the EDM's of certain alkali atoms might be several hundred times larger than that of the individual electron. Sandars termed this ratio of the atomic EDM to the electron EDM the enhancement factor. Sternheimer 6 later repeated Sandars' calculations and found enhancement factors that were in excellent agreement with those of Sandars'.

With Sandars' discovery of the enhancement effect, experiments could now be done

IT.D. Lee and C.N. Yang, Phys, Rev. 104, 254 (1956).

${ }^{2}$ L. Landau, Nucl. Phys. 3, 127 (1957).

${ }^{3}$ E. E. Salpeter, Phys. Rev. 112, 1642 (1958).

${ }^{4}$ L. I. Schiff, Phys. Rev. 132, 2194 (1963).

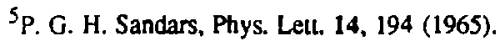

${ }^{6}$ R. M. Sternheimer, Phys. Rev. 183, 112 (1969). 
to measure the EDM's of atoms. The results could be directly interpreted as measurements of the electron EDM.

By 1970, three atomic EDM experiments were complete.7,8,9 All three used an atomic beam in an magnetic resonance type experiment. They did not find an electron EDM; instead they established a new limit: $d_{e}<3 \times 10^{-24} \mathrm{e}-\mathrm{cm}$.

There are currently two other groups searching for the electron EDM. The first, headed by E. Norval Fortson, at the University of Washington, Seattle, is working with cells of xenon, mercury, and rubidium gases. The other, L. Hunter and his students, at Amherst College, has achieved an improved measurement of $d_{e}$. By optically pumping a cell of cesium, Hunter ${ }^{10}$ has purportedly attained a limit of $d_{e}<7 \times 10^{-26} \mathrm{e}-\mathrm{cm}$.

Finally, a word should be said about the neutron EDM. The current limit is roughly $d_{n}<2 \times 10^{-25}$ e-cm. ${ }^{11}$ It should be noted that the neutron EDM is sensitive to CP violation in the quark sector, whereas the electron EDM depends on $\mathrm{CP}$ violation in the lepton sector. Therefore tests for the existence of $d_{e}$ and $d_{n}$ are complementary, not redundant.

\footnotetext{
${ }^{7}$ M. C. Weisskopf et al., Phys. Rev. Lea. 21, 1645 (1968).

${ }^{8}$ H. Gouid, Phys. Rev. Lett 24, 1091 (1970).

${ }^{9}$.M. A. Player and P. G. H. Sandars, J. Phys. B3, 1620 (1970).

${ }^{10}$ private communication with Larry Hunter.

${ }^{11}$ Unofficial repons. see also I. S. Altarev ef al., Phys. Lett. 102B, 13 (1981).
} 


\section{The Theory}

\section{A. The CP Violation Puzzle}

\section{The CP Symmetry}

$\mathrm{CP}$ is the product of charge conjugation $(\mathrm{C})$ and spatial inversion $(\mathrm{P})$. Although $\mathrm{C}$ violation and $\mathrm{P}$ violation occur in ordinary weak interactions, $\mathrm{CP}$ violation has been observed in only one system - the decay of neutral kaons. It was first seen by Cronin, Fitch, Christenson, and Turlay. 12 Although, this system has been studied extensively over the last twenty-five years, only very recently advances have been made toward a better understanding of the cause of $\mathrm{CP}$ violation.

\section{Baryon Asymmetry in the Universe}

Another fascinating aspect of the CP puzzle is the cosmological asymmetry between matter and anti-matter. Today it appears that the universe is composed principally of matter and very little anti-matter. It is possible to quantify this asymmetry. The ratio of the observed baryon density $n_{B}$ to the photon density $n_{\gamma}$ in today's universe is

$$
\frac{\mathrm{n}_{\mathrm{B}}}{\mathrm{n}_{\gamma}} \approx 3 \times 10^{-10}
$$

According to the standard cosmological model, after the universe had cooled sufficiently, baryon anti-baryon annihilation fell out of equilibrium with the expanding photon gas; this is called baryon freeze-out. The remnant baryon density $n_{B}$ indicates an initial (before freeze-out) baryon (quark) asymmetry of

$$
\frac{n_{\mathrm{q}}-n_{\bar{q}}^{-}}{n_{q}+n_{\bar{q}}^{-}}=10^{-9}
$$

12 J. H. Christenson et sh., Phys. Rev. Lett. 13, 138 (1964). 
What created this asymmetry? If we assume that the universe started in a symmetric state, some process must have occurred that violates baryon number (B), C, ana $\mathrm{CP}$. The need for $B$ violation and $C$ violation is quite clear; the need for $C P$ violation is subtle.

To understand it, consider the decay of baryons and antibaryons through some unknown interaction. If it were odd under $\mathrm{C}$ but even under $\mathrm{CP}$, then summing over al! spins and directions of the outgoing particles would yield the same decay rate for the particle and anti-particle. Thus, $\mathrm{CP}$ violation is required.

There are interesting SU(5) models that attempt to explain this $\mathrm{B}$ and $\mathrm{CP}$ violating interaction. ${ }^{i 3}$ They typically involve extremely heavy intermediate bosons (such as the $\mathrm{X}^{\mu}$ ) that would have been much more abundant when the universe was hot. ${ }^{14}$ In today's cooler universe, these bosons are scarce, but their presence might be manifested in any $\mathrm{CP}$ violating effects.

\section{The CPT Theorem}

The very important CPT theorem states that any Lorentz invariant local field theory is invariant under the combined operations of $\mathrm{C}, \mathrm{P}$, and time-reversal (T). Let $L$ be the Lagrangian describing some field theory. It can be proved that $L$ is invariant under CPT if the following requirements are satisfied 15

\section{1. $L$ is Lorentz invariant and local}

2. $L$ is nomally ordered

\section{3. $L$ is Hermitian}

We are principally concerned with the CPT theorem because it states that $\mathrm{CP}$ violation and $\mathrm{T}$ viclation are the same for any reasonaible field theory. In other words, $\mathrm{CP}$ is

\footnotetext{
${ }^{13}$ M. Yoshimura, Phys. Rev. Lett. 41, 281 (1978).

${ }^{14}$ A. D. Sakharov, Sov. Fhys. JETP Lett. 5, 24 (1967).

${ }^{15} \mathrm{M}$. Suzuki, Fhysics 225B leciure notes, p. 1.30, UC Berkeley.
} 
violated if and only if $\mathrm{T}$ is violated. Henceforth, we will interchange $\mathrm{CP}$ and $\mathrm{T}$ freely without any desire to distinguish between the two.

\section{CP Violation in the Standard Model}

What does it take for a theory to account for $\mathrm{CP}(\mathrm{T})$ violation? Let us write an amplinude $M$ as

$$
M=A f\left(\gamma^{\prime} s, p^{\prime} s\right)+B g\left(\gamma^{\prime} s, p^{\prime} s\right)
$$

where $f$ and $g$ are distinct combinations of the Dirac matrices and the momenta in the problem. If $\mathrm{A}$ and $\mathrm{B}$ are not relatively real, then $\mathrm{CP}$ is violated. This follows directly from the anti-unitary nature of time-reversal.

By incorporating a phase in the six-quark KM matrix, the standard model elegantly explains CP violation in the quark sector. ${ }^{16,17}$ The hadronic charged weak current is

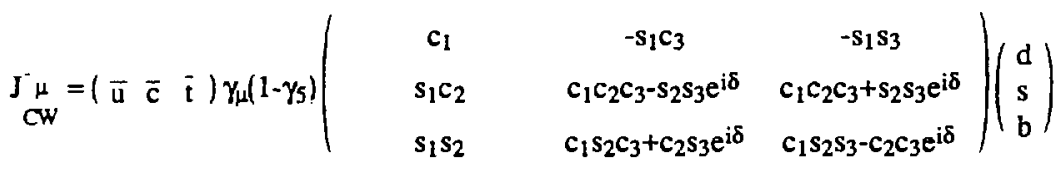

where $\delta$ is the CP violating phase and the c's and the s's are the cosines and sines of $\theta_{1}$, $\theta_{2}$, and $\theta_{3}-$ the generalized Cabbibo angles.

This approach has proven extremely successful in explaining the $\mathrm{K}^{0}-\overline{\mathrm{K}^{0}}$ mixing and the direct decays of $\mathrm{K}^{0} \rightarrow \pi$ 's.${ }^{18,19}$ The $\mathrm{K}^{0}-\overline{\mathrm{K}^{0}}$ mixing is calculate from Feynman

\footnotetext{
${ }^{16}$ M. Kobayashi and T. Maskawa, Prog. Theo. Phys. 49. 552 (1973).

${ }^{17}$ E. D. Commins and P. H. Bucksbaum, Weak Interactions of Leptons and Quarks. (Cambridge University Press, Cambridge, 1983), p 277.

${ }^{18} \mathrm{H}$. Burkhardt et al.(CERN, Dortmund, Edinburgh, Mainz, Orsay, Pisa, Siegen collab.) Phys. Leth B 206, 169 (1988).

${ }^{19}$ T. Ellis, M.K. Gaillard, D.V. Nanopoulos, Nucl. Phys. B 109, 213 (1976).
} 
diagrams of the form:

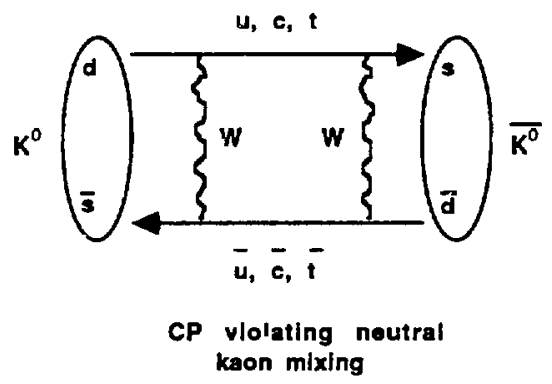

\section{Figure II.1}

whereas direct decays of $\mathrm{K}^{0} \rightarrow \pi$ 's originate in so called "penguin diagrams":

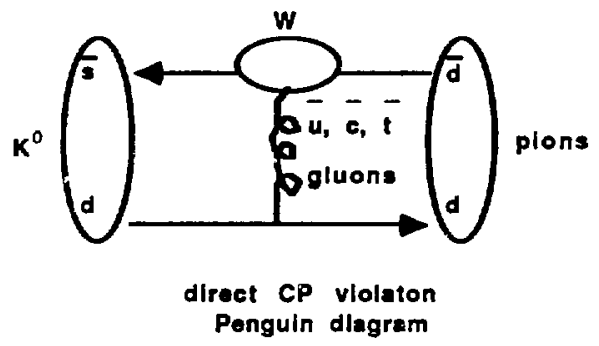

Figure II.2

When we sum these diagrams over the up, charmed, and top quarks, we have a sum of amplitudes that are not all relatively real. This will violate $\mathrm{CP}$. Since it is possible to distinguish between the two effects experimentally, both rates have been measured, and results agree reasonably well with the predictions of the standard model. 
In the leptonic sector, on the other hand, the standard model predicts extremply small or even no $\mathrm{CP}$ violation at ail. If all neutrinos are massless, then the mass eigenstates are trivially identical to the weak eigenstates. The leptonic charged weak current would simply be

$$
I_{C W}^{-}=\left(\overline{v_{e}} \overline{v_{\mu}} \overline{v_{\tau}}\right) \gamma_{\mu}\left(1-\gamma_{S}\right)\left(\begin{array}{lll}
1 & 0 & 0 \\
0 & 1 & 0 \\
0 & 0 & 1
\end{array}\right)\left(\begin{array}{c}
e^{-} \\
\mu^{-} \\
\tau
\end{array}\right)
$$

thus excluding any possible phase factors.

The current bounds on neutrino masses still leave open the possibility of a non-trivial KM matrix. The curre 1 it experimental masses are 20

$$
\begin{aligned}
m_{v_{\varepsilon}} & <46 \mathrm{eV} \\
m_{v_{\mu}} & <250 \mathrm{keV} \\
m_{v_{\varepsilon}} & <70 \mathrm{MeV}
\end{aligned}
$$

Assuming all neutrinos are relativistic when they decouple, measurements of the Hubble constant give an even stricter, cosmological limit on the neutrino masses: ${ }^{21}$

$$
\sum_{i} m_{v_{i}} \leq 80 \mathrm{eV}
$$

20 "Review of Particle Properties", Phys. Lett. 170B (1986).

2! L. Hall, Physics 250 lecture notes, p. 28, UC Berkeley. 


\section{B. The EDM of the Electron}

\section{A Non-Zero Electron EDM Violates CP Invariance}

Before turning to the various theories that make predictions about the electron EDM $\mathrm{d}_{e}$, let us demonsurate that a non-zero $\mathrm{d}_{e}$ really does violate $\mathrm{CP}$. The following argument applies to any elementary charged fermion. We begin with the Dirac Lagrangian for a fermion in an electromagnetic field. It turns out that there is only one unitary, Lorentz invariant, gauge invariant way to introduce an electric dipole moment:

$$
L=\bar{\psi}\left(\partial+\mathrm{m}-\mathrm{e} A+\frac{\mathrm{id}}{2} \gamma_{5} \sigma_{\mu v} F^{\mu v}\right) \psi+\text { h.c. }
$$

We have neglec:ed the anomalous magnetic dipole moment term for simplicity. In this section we convert this addition to the Lagrangian into a perturbing Hamiltonian $H_{\mathrm{EDM}}$. See Appendix A for a manifestly Lorentz invariant treatment of the $C, P$, and $T$ symmetries of the electron EDM.

The perturbing Hamiltun is

$$
H_{\text {EDM }}=\frac{i}{2} \mathrm{~d}_{e} \gamma_{0} \gamma_{5} \sigma_{\mu v} F^{\mu v}=\frac{i}{2} d_{e} \gamma_{0} \gamma_{5}\left(2 \sigma_{i 0} F^{i 0}+\sigma_{i j} F^{i j}\right)
$$

Manipulating the Dirac matrices, give us

$$
\sigma_{i 0} F^{i 0}=-\frac{i}{2}\left[\gamma_{i}, \gamma_{0}\right] E^{i}=-i \gamma_{i} \gamma_{0} E^{i}
$$

and

$$
\begin{aligned}
& \sigma_{i j}=\frac{i}{2}\left[\left(\begin{array}{cc}
0 & -\sigma_{i} \\
\sigma_{i} & 0
\end{array}\right)\left(\begin{array}{cc}
0 & -\sigma_{j} \\
\sigma_{j} & 0
\end{array}\right)-\left(\begin{array}{cc}
0 & -\sigma_{j} \\
\sigma_{j} & 0
\end{array}\right)\left(\begin{array}{cc}
0 & -\sigma_{i} \\
\sigma_{i} & 0
\end{array}\right)\right](
\end{aligned}
$$

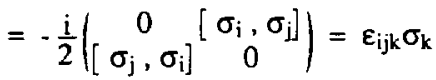

The EM field strength rensor is

$$
F^{i j}=-E_{i j k} B^{k}
$$

Putring this altogether, we have 


$$
\begin{aligned}
H_{\text {EDM }} & =\frac{i}{2} d_{e} \gamma_{0} \gamma_{5}\left(-2 \mathrm{i} \gamma_{i} \gamma_{0} \mathrm{E}^{\mathrm{i}}-\sigma_{\mathrm{k}} \mathrm{B}^{\mathrm{k}}\right) \\
& =\mathrm{d}_{\mathrm{e}} \gamma_{0}\left(\gamma_{5} \gamma_{\mathrm{i}} \gamma_{0} \mathrm{E}^{\mathrm{i}}-\frac{\mathrm{i}}{2} \gamma_{5} \sigma_{\mathrm{k}} \mathrm{B}^{\mathrm{k}}\right)
\end{aligned}
$$

which, simplifying the Dirac matrices, yields the final form:

$$
H_{\mathrm{EDM}}=\mathrm{d}_{\mathrm{e}} \gamma_{0}\left(\vec{\sigma} \cdot \overrightarrow{\mathrm{E}}+\frac{\mathrm{i}}{2} \gamma_{0} \vec{\gamma} \cdot \overrightarrow{\mathrm{B}}\right)
$$

Let us discuss the transformation properties of (II.B.8). First, $H_{\mathrm{EDM}}$ is odd under parity:

$$
\begin{array}{ll}
\mathrm{P} \overrightarrow{\vec{\sigma}} \mathrm{P}^{-1}=+\vec{\sigma} & \mathrm{P} \vec{\gamma} \mathrm{P}^{-1}=-\vec{\gamma} \\
\mathrm{P} \mathrm{E} \mathrm{P}^{-1}=-\overrightarrow{\mathrm{E}} & \mathrm{P} \overrightarrow{\mathrm{B}} \mathrm{P}^{-1}=+\overrightarrow{\mathrm{B}} \\
\mathrm{P} \gamma_{0} \mathrm{P}^{-1}=+\vartheta_{0} &
\end{array}
$$

and odd under time-reversal:

$$
\begin{array}{rlrl}
\mathrm{T} \vec{\sigma} \mathrm{T}^{-1} & =-\vec{\sigma} & \mathrm{T} \vec{\gamma} \mathrm{T}^{-1} & =-\vec{\gamma} \\
\mathrm{T} \overrightarrow{\mathrm{E}} \mathrm{T}^{-1} & =+\overrightarrow{\mathrm{E}} & \mathrm{T} \overrightarrow{\mathrm{B}} \mathrm{T}^{-1} & =-\overrightarrow{\mathrm{B}} \\
\mathrm{T} \gamma_{0} \mathrm{~T}^{-1} & =+\gamma_{0} & \mathrm{~T} \mathrm{i} \mathrm{T} & =-\mathrm{i}
\end{array}
$$

but even under charge coniugation:

$$
\begin{array}{llrl}
\mathrm{C} \vec{\sigma} \mathrm{C}^{-1}=+\vec{\sigma} & \mathrm{C} \vec{\gamma} \mathrm{C}^{-1}=-\vec{\gamma} \\
\mathrm{CE} \mathrm{C}^{-1}=-\overrightarrow{\mathrm{E}} & \mathrm{C} \overrightarrow{\mathrm{B}} \mathrm{C}^{-1}=-\overrightarrow{\mathrm{B}} \\
\mathrm{C} \gamma_{0} \mathrm{C}^{-1}=-\gamma_{0} & &
\end{array}
$$

We see that a non-zero $d_{e}$ violates $T$ and $P$, but conserves $C$, thus satisfying the CPT theorem.

\section{Predicted Electron EDM's for a Variety of Field Theories}

We consider five types of models for the electron EDM:

a. the Standard Model

b. a "non-minimal" Standard Model with heavy neutrinos

c. a hypothetical model with horizontal interactions

d. a left-right symmetric model

e. supersymmetric models 
The static electron EDM is the CP violating amplitude for the "dressed" electronphoton vertex in the limit where the photon momentum $k \rightarrow 0$. That is, $d_{e}$ is the coefficient in front of the $\gamma_{5} \sigma_{\mu \nu} F^{\mu v}$ term in the amplitude for the diagram:

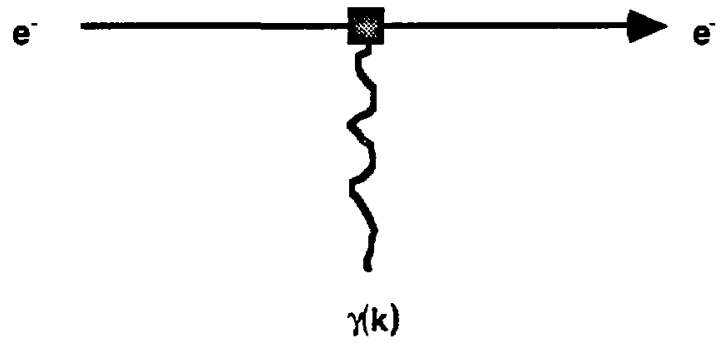

The electric dipole moment vertex

\section{Figure II.3}

The shaded square contains the $\mathrm{CP}$ violating intricacies of the field theory.

\section{a. The Standard Model}

As we have seen, if all neutrinos are massless, then there is no $\mathrm{CP}$ violation in the lepton sector and $d_{e}=0$. However, if the neutrinos hava non-zero masses somewhere below the current experimental limits, $d_{e} \neq 0$, but it is very small. The lowest order contribution to $d_{e}$ in the standard model is the 3-loop diagram 22 


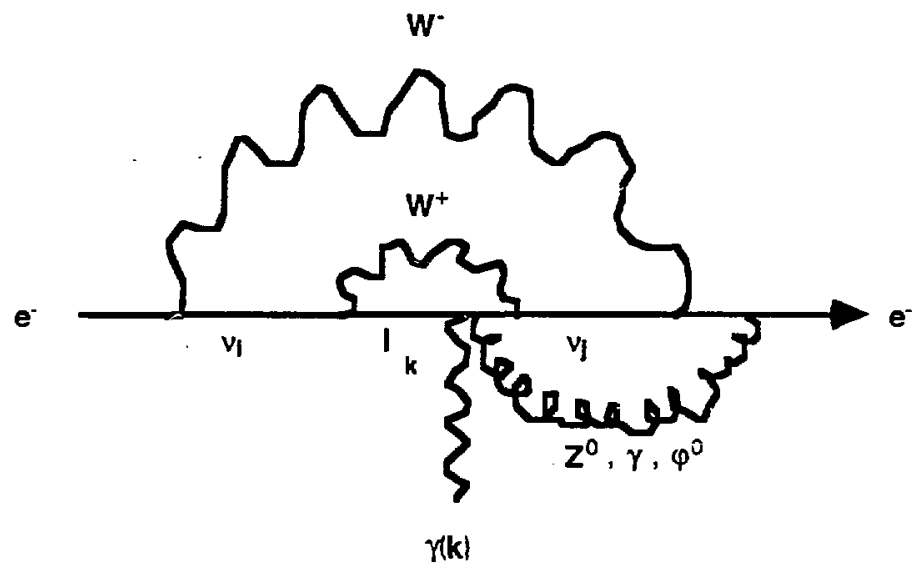

\section{Lowest order contribution to EDM in the standard model}

\section{Figure II.4}

where the $v_{i}$ and $v_{j}$ are two different neutrinos, $l_{k}$ is some intermediate lepton, and the neutral (curly) line is a $Z^{0}, \gamma$, or a $\phi^{0}$ (a neutral Higgs particle). The two $W^{ \pm}$loops provide the necessary $\mathrm{CP}$ violating phase. The additional curly line requires an explanation. It was once thought that a static de could arise at the order $G_{F}{ }^{2}$ (two W土 loops). However, Shabalin ${ }^{23}$ noticed that there is complete cancellation for the 2-loop diagrams in the $\mathrm{k} \rightarrow 0$ limit. One more intemal line is needed to upset this cancellation.

To calculate $d_{e}$, we would have to write down all twenty or thiry 3-loop diagrams of this kind. We would include all possible positions of the external photon, all possible intermediate particles, and all possible positions of the neutral interaction. We would then isolate the terms proportional to $\gamma_{5} \sigma_{\mu \nu} k^{\mu} \varepsilon^{v}$ ( $k^{\mu}$ and $\varepsilon^{v}$ are the external photon momentum and polarization respectively) and integrate over all intersal 4-momenta. Finally, we would sum the diagrams and take the static limit $d_{e} \rightarrow 0$. This is a lot of work.

E. P. Shabalin, Sov. J. Nucl. Phys. 28, 75 (1978). 
In lieu of actually calculating $\mathrm{d}_{\mathrm{e}}$, we can do an order-of-magnitude estimation and hope there are no unforeseen cancellations. To make matters clear, let us consider a particular diagram like the one above with an internal photon (not $Z^{0}$ or $\phi^{0}$ ) and employ units $\hbar=c=1$.

First, $d_{e}$ is independent of all the momenta in the problem because we are taking the static limit. In addition to 2 intermediate $W \pm$ bosons, there are 4 weak and 3 electromagnetic vertices. This suggests

$$
d_{e}=\frac{g^{4} e^{3}}{\pi^{4} m_{W}^{4}} f\left(m_{v_{\tau}}, m_{v_{\mu}}, m_{k}, m_{e}\right)
$$

where we have included a factor $f$ to account for the various mixings in the leptonic KM matrix as well as internal propagators. Using $e=g \sin \theta_{W}$ and $\alpha=e^{2}$, we can rewrite (II.B.12) as,

$$
\mathrm{d}_{\mathrm{e}}=\frac{\mathrm{e} \mathrm{G}_{\mathrm{F}} \alpha^{2}}{\pi^{4} \mathrm{~m}_{\mathrm{W}}^{4} \sin ^{2} \mathrm{~g}_{\mathrm{W}}} \mathrm{f}\left(\mathrm{m}_{\mathrm{v}_{\mathrm{r}}}, \mathrm{m}_{\mathrm{V}_{\mathrm{\mu}}}, \mathrm{m}_{\mathrm{k}}, \mathrm{m}_{\mathrm{e}}\right)
$$

By summing over diagrams, GIM suppression factors will appear in $f$ in a form first suggested by Donoghue 24

$$
f \approx \frac{\left(m_{\tau}^{2}-m_{\mu}^{2}\right)\left(m_{v_{\tau}}^{2}-m_{v_{\mu}}^{2}\right)\left(m_{v_{r}}^{2}-m_{v_{e}}^{2}\right)\left(m_{v_{\mu}}^{2}-m_{v_{\tau}}^{2}\right) m_{e}}{m_{\tau}^{4}}
$$

Clearly, the amount of $\mathrm{CP}$ violation should depend on the mass splittings, not just the neutrino masses. Using $m_{e} G_{F} \alpha=10^{-24} \mathrm{~cm}$, we find that the standard model predicts an electron EDM of order

$$
\mathrm{d}_{\mathrm{e}} \approx 10^{-35}\left[\Delta \mathrm{m}_{\mathrm{v}}(\mathrm{GeV})\right]^{3} \mathrm{e}-\mathrm{cm}
$$

We emphasize that this is only a crude order of magnitude argument. If we use the current cosmological limits on the neutrino masses $\left(\Delta \mathrm{m}_{\mathrm{V}} \approx 100 \mathrm{eV}\right)$ we find

$$
\mathrm{de}_{\mathrm{e}} \leq 8 \times 10^{-56} \mathrm{e}-\mathrm{cm}
$$

which is well beyond any experimental reach.

24J. Donoghue, Phys. Rev. D. 18, 1632 (1978). 


\section{b. A "Non-Minimal" Standard Model with New Heavy Neutrinos}

If the standard model is extended to include two new generations of leptons with neutrino mass splittings on the order of $\approx \mathrm{m}_{W}$, the last section suggests that $\mathrm{d}_{\mathrm{e}}$ can be as large as

$$
\mathrm{d}_{\mathrm{e}} \leq 5 \times 10^{-30} \mathrm{e}-\mathrm{cm}
$$

\section{c. A Hynothetical_Model with_Korizontal_Interactions}

Many theories of current interest include lepton-lepton couplings (dileptons), leptonquark couplings (leptoquarks), and quark-quark couplings (diquarks). GUTS/SU(5) models 25 , "superstring-inspired" modeis ${ }^{26}$, and further phenomenological extensions of the standard model 27 are sorne of these. In these theories, CP violation can occur at the one-loop level because there are generally different right-handed and left-handed complex couplings. Their predictions for $\mathrm{d}_{e}$ are principally constrained by measurements of $\mu \rightarrow$ ey.

For demonstrative purposes, we concentrate on an extension of the standard model with a hypothetical scalar leptoquark $\phi$ which transforms as $(3,2,7 / 6)$ under $S U(3)_{C} \otimes S U(2)_{L} \otimes U(1)_{Y} .28$ This particle has Yukawa type couplings between leptons and quarks. Let us focus on the couplings to the electron. The Lagrangian is

$$
L=\lambda_{R} \phi \overline{q_{i R}} e_{L}+\lambda_{L} \phi \overline{q_{i L}} e_{R}+\text { h.c. }
$$

\footnotetext{
${ }^{25}$ Tai-Pei Cheng and Ling-Fong Li, Gauge Theory of Elemenry Particle Physics (Oxford University Press, New York, 1984), p. 442.

${ }^{26}$ A. Barroso and J. Maalampi, Phys. Lex. B 187, 85 (1987).

27 A. Zee, Phys. Rev. Lell. 55, 2382 (1985).

${ }^{28}$ S. M. Barr and A. Masiero, Phys. Rev. Lett. 58, 187 (1987).
} 
where $\mathrm{q}_{i}=u, c, t$. Notice that if we include a helicity flip of the intermediate quark, $C P$ is violated because $\lambda_{R}^{*} \lambda_{L}$ is not necessarily real. A typical diagram might be

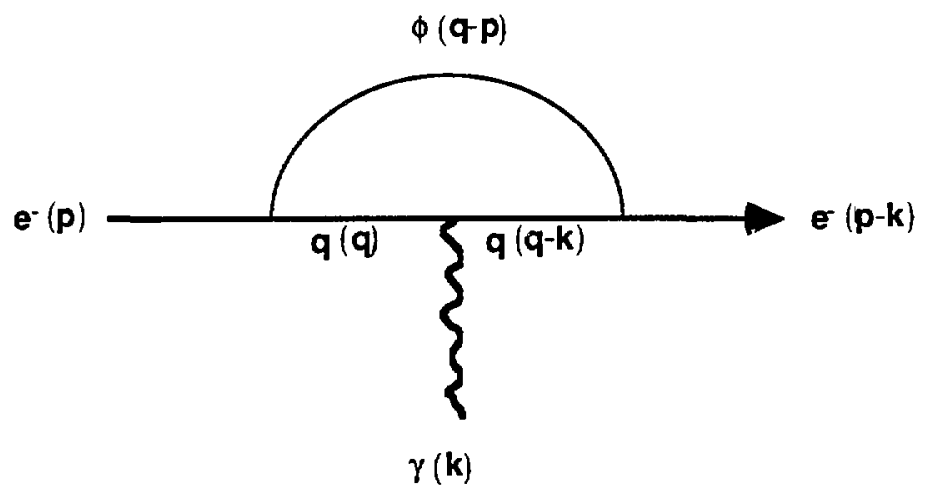

\section{Scalar leptoquark contribution to electron EDM}

Figure II.5

In Appendix B we calculate this Feynman diagram exactly and find in the static linint:

$$
\mathrm{d}_{e} \leq \frac{\operatorname{Re}\left(\lambda_{\mathrm{R}}^{*} \lambda_{\mathrm{L}}\right)}{24 \pi^{2}} \frac{\mathrm{m}_{\mathrm{q}}}{\mathrm{m}_{\phi}^{2}}\left(1+\ln \left(\frac{\mathrm{m}_{\mathrm{q}}^{2}}{\mathrm{~m}_{\phi}^{2}}\right)\right)
$$

The same process that would generate a non-zero electron EDM also would contribute to the the lepton number violating decay $\mu \rightarrow e \gamma$. This can be seen by replacing the incoming electron line with a muon line. Using upper limit on the branching ratio for $\mu \rightarrow e \gamma$ and working backward, we find an upper limit on $d_{e}$ for these models:

$$
d_{e} \leq 3 \times 10^{-26} \mathrm{e}-\mathrm{cm}
$$




\section{d. A Left-Right_Summetric_Model}

The left-right symmetric model allows an electron EDM as large as the current experimental limit. 29 This theory posits the existence of right-handed charged weak currents as well as left-handed and includes separate particles $\mathrm{W}_{\mathrm{L}}, \mathrm{W}_{\mathrm{R}}, \mathrm{v}_{\mathrm{L}}$, and $\mathrm{v}_{\mathrm{R}}$. If the right-handed $W_{R} \pm$ boson is at least 3-4 times more massive than its left-handed counterpart $\mathrm{W}_{\mathrm{L}^{ \pm}}$, no inconsistencies can be found with observations. The electron Lagrangian is

$$
L=\frac{g}{2 \sqrt{2}}\left(w_{1}^{\mu} \bar{e}_{L} \gamma_{\mu} v_{e L}+w_{2}^{\mu} \bar{e}_{R} \gamma_{\mu} v_{e R}\right)+\text { h.c. }
$$

The mass eigenstates $W_{L}$ and $W_{R}$ are related to the gauge eigenstates $W_{1}$ and $W_{2}$ by

$$
\left(\begin{array}{l}
W_{L} \\
W_{R}
\end{array}\right)=\left(\begin{array}{cc}
\cos \chi & \sin \chi \\
-\sin \chi & \cos \chi
\end{array}\right)\left(\begin{array}{l}
W_{1} \\
W_{2}
\end{array}\right)
$$

and similarly for the neutrinos:

$$
\left(\begin{array}{l}
v_{L} \\
v_{R}
\end{array}\right)=\left(\begin{array}{cc}
\cos \zeta & \sin \zeta \\
-\sin \zeta & \cos \zeta
\end{array}\right)\left(\begin{array}{l}
v_{1} \\
v_{2}
\end{array}\right)
$$

An EDM would arise through $\mathrm{W}_{\mathrm{L}}-\mathrm{W}_{\mathrm{R}}$ and $v_{\mathrm{L}}-\mathrm{v}_{\mathrm{R}}$ oscillations with a complex phase originating at the two charged-weak vertices. 


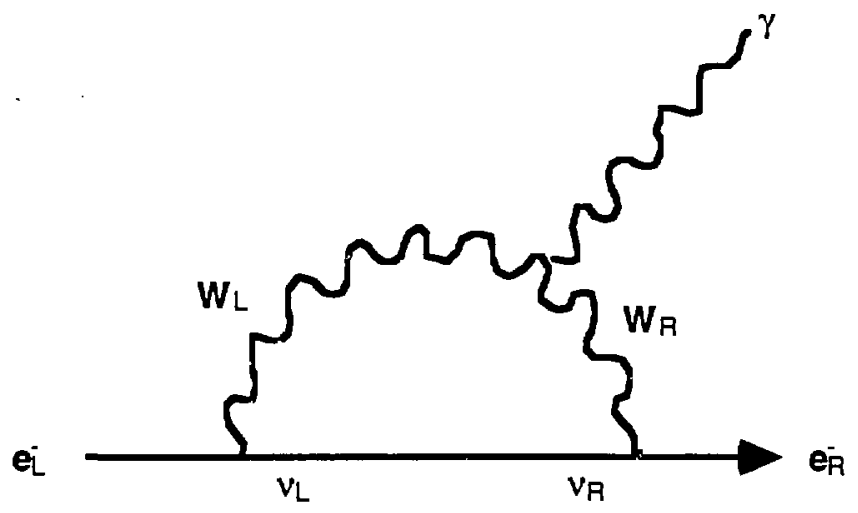

\section{Lowest order contribution} to de in a left-right symmetric theory

\section{Figure II.6}

Because little is known about the mass of the right handed neutrino, $d_{e}$ is unconstrained:

$$
\text { de } 5 \text { current exp. limit }
$$

\section{e. Supersymmetric Models}

Supersymmetric theories provide ma " channels for the possibility of CP. violation. ${ }^{30}$ We do not elaborate, but simply quote a limit on $d_{e}$ of

$$
d_{e} \leq 10^{-27} \mathrm{e}-\mathrm{cm}
$$

We conclude with a table summarizing the predictions of the various theories.

${ }^{30}$ M. B. Gavela, "CP-violation in supersymmetric theories", in Theoretical Symposium on Insense Medium Energy Sources of Sirangeness, University of California at Santa Cruz, 1983, edited by T. Goldman, H. E. Haber, and H. F. W. Sadronzinski (AIP, New York, 1983), Vol. 102. 
Predictions for EDM from various theories

\begin{tabular}{|c|c|}
\hline theory & Jimit \\
\hline standard model & $\mathrm{d} \cdot \leq 10^{-55} \mathrm{ecm}$ \\
\hline $\begin{array}{l}\text { standard model } \\
\text { w/ heavy neutrinos }\end{array}$ & $\mathrm{d}_{v} \leq 10^{-35}\left(\Delta_{\mathrm{mv}}\right)^{3} \mathrm{ecm}$ \\
\hline horizontal models & $\mathrm{d} \cdot \leq 10^{-26} \mathrm{ecm}$ \\
\hline $\begin{array}{l}\text { left-right symmetric } \\
\text { models }\end{array}$ & $\begin{array}{l}\text { current exp. } \\
\text { limit }\end{array}$ \\
\hline SUSY models & d. $\leq 10^{-27} \mathrm{ecm}$ \\
\hline
\end{tabular}

Figure 11.7 


\section{The EDM of the Atom}

\section{Schiff's Theorem}

Because we measire the atomic EDM $d_{\text {atom }}$ instead of the electron EDM delectron, we need to establish a definite relationship between the two. To facilitate this, we define an enhancement factor $\mathrm{R}$ :

$$
\mathrm{R}=\frac{\mathbf{d}_{\text {atom }}}{\mathrm{d}_{\text {electron }}}
$$

It turns out that $\mathrm{R}$ can be much greater than unity for certain atoms. This enhancement process has no simple physical explanation. Instead of suggesting an erroneous, oversimplified model, we will derive it by examining the quantum mechanics of a relativistic many-electron atom.

We first discuss a reiativistic multi-electron atom consisting of electrons with electric dipole moments $\mathrm{d}_{e}$. Then we show that a non-zero atomic EDM is a purely relativistic effect (Schiff's theorem) and work the expression for $\mathbf{R}$ into a manageable form. Finally, we review numerical soltutions and summarize their results.

The unperturbed Hamiltonian for a relativistic many-electron atom is

$$
H_{0}=\sum_{i}\left(\vec{\alpha}_{i} \cdot \vec{p}_{i}+\beta_{i} m\right)+e V
$$

where $m$ is the mass of the electron and the atomic potential $V$ is

$$
V=-\sum_{i} \frac{e Z}{r_{i}}+\frac{1}{2} \sum_{i, j} \frac{e}{r_{i j}}
$$

The sums over $\mathrm{i}$ and $\mathrm{j}$ represent sums over all electrons. We have adopted the atomic physic notation of $\beta=\gamma_{0}$ and $\vec{\alpha}=\gamma_{0} \vec{\gamma}$. Also, $Z$ is the atomic number (nuclear charge) of the atom. 
We neglect the Breit interaction for simplicity. In the presence of an external electric field applied aiong the $z$-axis, we have a perturbation $H^{\prime}$ (See $\S$ II.B.1):

$$
H^{\prime}=-\sum_{\mathrm{j}}\left(\mathrm{d}_{\mathrm{e}} \beta_{\mathrm{j}} \vec{\sigma}_{\mathrm{j}} \cdot \overrightarrow{\mathrm{E}}_{\mathrm{j}}^{\mathrm{tot}}+\mathrm{ez}_{\mathrm{j}} \mathrm{E}^{\mathrm{ext}}\right)
$$

where $\vec{E}^{\text {tot }}$ is the total field seen by each electron,

$$
\overrightarrow{\mathrm{E}}^{\mathrm{tot}}=\mathrm{E}^{\mathrm{ext}}-\vec{\nabla}_{\mathrm{j}} \mathrm{V}
$$

This first order energy shift of the ground state $|0\rangle$ that is linear in the applied external electric field is

$$
\Delta F^{(1)}=-\left\langle 01 \sum_{j} d_{e} \beta_{j} \sigma_{2} E^{e x t} \mid 0\right\rangle
$$

Notice that this exhibits no enhancement; eqn. (II.C.6) is just the sum of the individual electron EDM's interacting with the electric field. However, we now show that this term is exactly cancelled by the non-relativistic part of the second order energy shift. The only term in the second order shift that is linear in Eext is

$$
\Delta E_{\text {lin }}^{(2)}=-\sum_{n} \frac{1}{E_{0}-E_{n}}\langle 0| \sum_{i} e z_{i} E^{e x t} \ln \left\langle n\left|\sum_{j} d_{e} \beta_{j} \vec{\sigma}_{j} \cdot \nabla_{j} v\right| 0\right\rangle+c . c .
$$

where the eigenstates $|\mathrm{n}\rangle$ of $H_{0}$ and the ground state 10$\rangle$ are of opposite parity. Now,

$$
\mathrm{e} \vec{\sigma}_{\mathrm{j}} \cdot \vec{\nabla}_{\mathrm{j}} \mathrm{V}=\left[\vec{\sigma}_{\mathrm{j}} \cdot \vec{\nabla}_{\mathrm{j}}, H_{0} \cdot \sum_{\mathrm{i}}\left(\vec{\alpha}_{\mathrm{i}} \cdot \overrightarrow{\mathrm{p}}_{\mathrm{i}}+\beta_{\mathrm{j}} \mathrm{m}\right)\right]
$$

Thus, we can rewrite $\Delta E_{\text {lin }}^{(2)}$ as:

$$
\begin{gathered}
\Delta \mathrm{E}_{\mathrm{lin}}^{(2)}=-\frac{1}{\mathrm{E}_{0}-\mathrm{E}_{\mathrm{n}}}\left\langle 0\left|\sum_{\mathrm{i}} \mathrm{z}_{\mathrm{i}} \mathrm{E}^{\text {ext }}\right| \mathrm{n}\right\rangle \\
\left\langle\mathrm{n}\left|\sum_{\mathrm{j}} \mathrm{d}_{\mathrm{e}}\left[\beta_{\mathrm{j}} \vec{\sigma}_{\mathrm{j}} \cdot \vec{\nabla}_{\mathrm{j}}, H_{0}-\sum_{\mathrm{k}}\left(\vec{\alpha}_{\mathrm{k}} \cdot \overrightarrow{\mathrm{p}}_{\mathrm{k}}+\beta_{\mathrm{k}} \mathrm{m}\right)\right]\right| 0\right\rangle \\
+ \text { c.c. }
\end{gathered}
$$

It is instructive to separate eqn. (II.C.9) into relativistic and non-relativistic parts. Consider first the non-relativistic (diagonal) term proportional to $H_{0}$. We know that the commutator of any operator $O$ with the unperturbed Hamiltonian $H_{0}$ is

$$
\left\langle\mathrm{n}\left|\left[O, H_{0}\right]\right| 0\right\rangle=\left(\mathrm{E}_{0}-\mathrm{E}_{\mathrm{n}}\right)\langle\mathrm{n}|O| 0\rangle
$$


Using this to cancel the commutator and the energy denominator, we find

$$
\Delta \mathrm{E}_{\text {non-rel }}^{(2)}=-\sum_{\mathrm{n}}\langle 0| \sum_{\mathrm{i}} \mathrm{z}_{\mathrm{i}} \mathrm{E}^{\mathrm{ext}} \ln \left\langle\mathrm{n}\left|\sum_{\mathrm{j}} \mathrm{d}_{\mathrm{e}} \beta_{\mathrm{j}} \vec{\sigma}_{\mathrm{j}} \cdot \vec{\nabla}_{\mathrm{j}}\right| 0\right\rangle+\text { c.c. }
$$

Employing the completeness of states to remove the sum over $n$, and adding the complex conjugate to (II.C.11), we obtain

$$
\underset{\text { non-rel }}{\Delta \mathrm{E}_{\text {lin }}^{(2)}}=-\left\langle 01\left[\left(\sum_{\mathrm{i}} \mathrm{z}_{\mathrm{i}} \mathrm{E}^{\mathrm{ext}}\right) \cdot\left(\sum_{\mathrm{j}} \mathrm{d}_{\mathrm{e}} \beta_{\mathrm{j}} \vec{\sigma}_{\mathrm{j}} \cdot \nabla_{\mathrm{j}}\right)\right] 10\right\rangle
$$

This vanishes unless $i=j$, when

$$
\underset{\text { non-rel }}{\Delta \mathrm{E}_{\text {iin }}^{(2)}}=-\left\langle 0\left|\mathrm{~d}_{e} \sum_{\mathrm{i}} \beta_{\mathrm{i}} \vec{\sigma}_{\mathrm{i}} \cdot\left[\vec{\nabla}_{\mathrm{i}}, z\right] \mathrm{E}^{\mathrm{ext}}\right| 0\right\rangle
$$

or

$$
\underset{\text { non-rel }}{\Delta E_{\text {lin }}^{(2)}}=\left\langle 0\left|\sum_{i} d_{e} \beta_{i} \sigma_{z} E^{e x t}\right| 0\right\rangle=-\Delta E_{\text {lin }}^{(1)}
$$

In other words, $\Delta \mathrm{E}_{\text {non-rel }}^{(2)}$ cancels $\Delta \mathrm{E}_{\text {lin }}^{(1)}$. Therefore, in the non-relativistic limit, the enegy shift of an atom in an applied electric field vanishes to second order even if $d_{e} \neq 0$. This is Schiff's theorem.

\section{Calculating the Enhancement Factor}

Thus, it is the remaining energy shift, the relativistic (off-diagonal) second-order term, that is responsible for the EDM enhancement. We now convert this awkward, many-body perurbation into a one-body operator. We saw that:

$$
\begin{gathered}
\Delta E^{(2)} \operatorname{lin}_{\text {relativistic }}=\frac{1}{E_{0}-E_{n}}\left\langle 0\left|\sum_{i} z_{i} E^{\text {ext }}\right| n\right\rangle \\
\left\langle n\left|\sum_{j} d_{e}\left[\beta_{j} \vec{\sigma}_{j} \cdot \vec{\nabla}_{j}, \sum_{k}\left(\vec{\alpha}_{k} \cdot \vec{p}_{k}+\beta_{k} m\right)\right]\right| 0\right\rangle+\text { c.c. }
\end{gathered}
$$

substituting $\vec{\nabla}=\mathrm{i} \overrightarrow{\mathrm{p}}$ into (II.C.15), we find

$$
\begin{gathered}
\Delta E^{(2)} \operatorname{lin}_{\text {relativistic }}=\frac{i d_{e}}{E_{0}-E_{n}}\left\langle 0\left|\sum_{i} z_{i} E^{e x t}\right| n\right\rangle \\
\left\langle n\left|\sum_{j k}\left[\beta_{j} \vec{\sigma}_{j} \cdot \vec{p}_{j},\left(\vec{\alpha}_{k} \cdot \vec{p}_{k}+\beta_{k} m\right)\right]\right| 0\right\rangle+\text { c.c. }
\end{gathered}
$$


The commutators vanish unless $j=k$. With a little work we find

$$
[\beta \vec{\sigma} \cdot \vec{p}, \vec{\alpha} \cdot \vec{p}]=-2 \gamma_{0} \gamma_{5} \vec{\sigma} \cdot \vec{p} \vec{\sigma} \cdot \vec{p}=-2 \gamma_{0} \gamma_{5} \vec{p}^{2}
$$

which can then be inserted into (II.C.16) to give the very simple form:

$$
\Delta \mathrm{E}_{\text {relativistic }}^{(2)}=-2 \mathrm{id}_{\mathrm{e}} \mathrm{E}^{\mathrm{ext}} \sum_{\mathrm{n}} \frac{1}{\mathrm{E}_{0}-\mathrm{E}_{\mathrm{n}}}\langle 0|\mathrm{z}| \mathrm{n}\rangle\left\langle\mathrm{n}\left|\gamma_{0} \gamma_{5} \overrightarrow{\mathrm{p}}^{2}\right| 0\right\rangle+\text { c.c. }
$$

We can now write down an extremely convenient expression for the enhancement factor R:

$$
R=\frac{\Delta E^{(2)} l_{\text {relativistic }}^{\text {in }}}{d_{e} E^{e x t}}=-2 i \sum_{n} \frac{1}{E_{0}-E_{n}}\langle 0 \mid z \ln \rangle\left\langle n\left|\gamma_{0} \gamma_{5} \vec{p}^{2}\right| 0\right\rangle+\text { c.c. }
$$

To evaluate this expression numerically, it must be further simplified.

First we will use the Wigner-Eckhart theorem to resolve any angular momentum factors. Since we are concerned mainly with alkali type atoms, we will assume each state |n) has well defined angular momenta $\left|j_{n}, l_{n}, m_{n}\right\rangle$. The first bra-ket in (II.C.19) is

$$
\langle 0|z| n\rangle=(-1)^{j_{0}-m_{0}}\left[\begin{array}{ccc}
-j_{0} & 1 & j_{n} \\
m_{0} & 0 & m_{n}
\end{array}\right] C_{1}(0, n)\langle 0\|r\| n\rangle
$$

where we have employed the 3-j symbols and used the definitions:

$$
C_{1}(0, n)=(-1)^{j_{0}+\frac{1}{2}} \sqrt{\left(2 j_{0}+1\right)\left(2 j_{0}+1\right)}\left[\begin{array}{rrr}
j_{0} & j_{n} & 1 \\
-\frac{1}{2} & +\frac{1}{2} & 0
\end{array}\right] \pi\left(l_{0}, l_{n}, 1\right)
$$

and

$$
\pi\left(l_{0}, l_{n}, 1\right)=\left\{\begin{array}{ll}
1 & \text { if } l_{0}+l_{n}+1 \text { is even } \\
0 \text { if } l_{0}+l_{n}+1 \text { is odd }
\end{array}\right\}
$$

This is advantageous because the reduced marrix element $\langle 0 \| r \mid n\rangle$ is just an integral over $r$. The next bra-ket in (II.C.19) is

$$
\left\langle\mathrm{n}\left|\gamma_{0} \gamma_{5} \overrightarrow{\mathrm{p}}^{2}\right| 0\right\rangle=\delta_{\mathrm{j}_{\mathrm{a}-j_{0}}} \delta_{\mathrm{m}_{\mathrm{a}}, \mathrm{m}_{0}}\left\langle\mathrm{n}\left\|\gamma_{0} \gamma_{5} \overrightarrow{\mathrm{p}}^{2}\right\| 0\right\rangle
$$

Insering this into our expression for $R$, we find

$$
\begin{gathered}
R=-2 i(-1)^{2 j_{0}-m_{0}+\frac{1}{2}}\left(2 j_{0}+1\right)\left[\begin{array}{ccc}
j_{0} & 1 & j_{0} \\
-m_{0} & 0 & m_{0}
\end{array}\right]\left[\begin{array}{ccc}
j_{0} & j_{0} & 1 \\
-\frac{1}{2} & \frac{1}{2} & 0
\end{array}\right] \\
\cdot \sum_{n} \frac{\left.\langle 0\|r\| n\rangle / n\left\|\gamma_{0} \gamma_{5} \vec{p}^{2}\right\| 0\right\rangle}{E_{0}-E_{n}}+c . c .
\end{gathered}
$$


We can go even further by defining the parity mixed state (mixed by the EDM perturbation) $)^{31}$

$$
\overline{0}\rangle=\sum_{\mathbf{n}} \frac{|\mathbf{n}\rangle\left\langle\mathbf{n}\left\|\gamma_{0} \gamma_{5} \overrightarrow{\mathbf{p}}^{2}\right\| 0\right\rangle}{E_{0}-E_{\mathbf{n}}}
$$

This formula can be expressed as a differential equation

$$
\left.\left(H_{0}^{\text {radial }}-\mathrm{E}_{0}\right) \overline{|0\rangle}=-\gamma_{0} \gamma_{5} \overrightarrow{\mathrm{p}}^{2} 10\right\rangle
$$

and we finally obtain,

$$
\begin{gathered}
\mathrm{R}=-2 \mathrm{i}(-1)^{2 \mathrm{j}_{0}-\mathrm{m}_{0}+\frac{1}{2}}\left(2 \mathrm{j}_{0}+1\right)\left[\begin{array}{ccc}
\mathrm{j}_{0} & 1 & \mathrm{j}_{0} \\
-\mathrm{m}_{0} & 0 & \mathrm{~m}_{0}
\end{array}\right]\left[\begin{array}{ccc}
\mathrm{j}_{0} & \mathrm{j}_{0} & 1 \\
-\frac{1}{2} & \frac{1}{2} & 0
\end{array}\right] \\
\cdot(\langle\overline{0}\|\mathrm{r}\| 0\rangle+\langle 0\|\mathrm{r}\| \overline{0}\rangle) \\
\text { where }\left(H_{0}^{\text {radial }}-\mathrm{E}_{0}\right)|\overline{0}\rangle=-\gamma_{0} \gamma_{5} \overrightarrow{\mathrm{p}}^{2}|0\rangle
\end{gathered}
$$

To summarize, we have derived a simple expression for the enhancement factor $\mathbf{R}$ (II.C.27), which involves a one-dimensional integral and an ordinary differential equation. Given the ground state radial wavefunction 10$\rangle, \mathrm{R}$ can be calculated on a personal computer.

Determining 10 ) is non-trivial because the valence electron sees a shielded potential and because electron-electron repulsion is a many-body problem. There are two distinct approaches to this problem.

One suprisingly effective method is to replace the many-body atomic potential $V$ with a model potential that can be "tuned" to yield results that agree with well known experimental quantities. 32,33 For example, Johnson et al. use a Tietz potential

${ }^{31}$ W. R. Johnson et al., Phys. Rev. A 34, 1043 (1986).

32 D. Neuffer and E. D. Commins, Phys. Rev, A 16, 1760 (1977) ; 16, 844 (1977).

33 W. R. Johnson et al., Phys. Rev. A 32, 2093 (1985). 


$$
V_{\text {Tiezz }}(r)=-\frac{\alpha}{r}\left[1+\frac{(Z-1)}{(1+t r)^{2}} e^{-\gamma}\right]
$$

to calculate the ground state 10$\rangle$. The parameters $\alpha, t$, and $\gamma$ are adjusted to fit the low-lying spectra. Enhancement factors determined in this way for a variety of different model potentials usually lie within 20 percent of each other.

The other method involves an a priori Hartree-Fock calculation followed by a few orders of many-body perturbative corrections. ${ }^{34}$ This technique is extremely difficult and often fails when the correction terms do not converge rapidly enough. Although the Hartree-Fock many-body approach is more fundamental, its results are generally no better than those obtained through the model potentials. This can be demonstrated by comparing predictions and measurements of parity violation in atoms. Let us summarize the enhancement factors obtained by Johnson et al.

\section{Table of enhancement factors}

\begin{tabular}{|c|c|c|c|c|}
\hline atom & $\begin{array}{l}\text { Tletz } \\
\text { potential }\end{array}$ & $\begin{array}{l}\text { Green } \\
\text { potential }\end{array}$ & $\begin{array}{l}\text { Noreross } \\
\text { potential }\end{array}$ & $\begin{array}{l}\text { Hartree-Fock } \\
\text { Ist orde: } \\
\text { MBPT }\end{array}$ \\
\hline Rb & 16 & 24 & 22 & 25 \\
\hline Cs & 80 & 106 & 100 & 115 \\
\hline Au & .60 & 131 & 135 & 250 \\
\hline TI & -502 & -607 & .562 & -1041 \\
\hline
\end{tabular}

Figure II.8

34 Sce, for example, I. Lindgren and J. Morrison, Alomic Many-Body Theory, (Springer-Verlag, Berlin, 1982). 
It is clear that thallium has the largest enhancement factor. Because of the large overlap and near degeneracy of the $6 \mathrm{p}$ and $6 \mathrm{~s}$ orbitals, the Hartree-Fock calculation does not converge rapidly. Ignoring it, we find an approximate thallium enhancement factor:

$$
R_{\text {thallium }}=\left(\frac{\mathrm{d}_{\text {atom }}}{d_{\text {elecron }}}\right)_{\text {thallium }}=-560 \pm 50
$$

Thallium is an ideal choice fur our electron electric dipole moment search. Not only does it have an exceptionally large enhancement factor, but it also is relatively easy to create an atomic beam of thallium using standard techniques. Furthermore, the thallium energy levels are very convenient for optical polarization and analysis. 


\section{The experiment}

\section{A. An Overview of the Technique}

The technique that we employ to measure the electric dipole moment of the thallium atom is both a familiar and well tested one. Using an atomic beam resonance apparatus with two separated radiofrequency regions (the Ramsey interference technique), we look for an energy shift of the $6 \mathrm{P}_{1 / 2}(\mathrm{~F}=1)$ levels that is linear in an applied external electric field. A reader who is unfamiliar with the Ramsey technique should see Appendix $\mathrm{C}$ which describes a model Ramsey magnetic resonance experiment.

Figure III.1 is an energy level diagram of thallium (not to scale). The two stable thallium isotopes are $\mathrm{T}^{205}(70 \%)$ and $\mathrm{Tl}^{203}(30 \%)$. We perform our experiment on the more abundant $\mathrm{Tl}^{205}$.

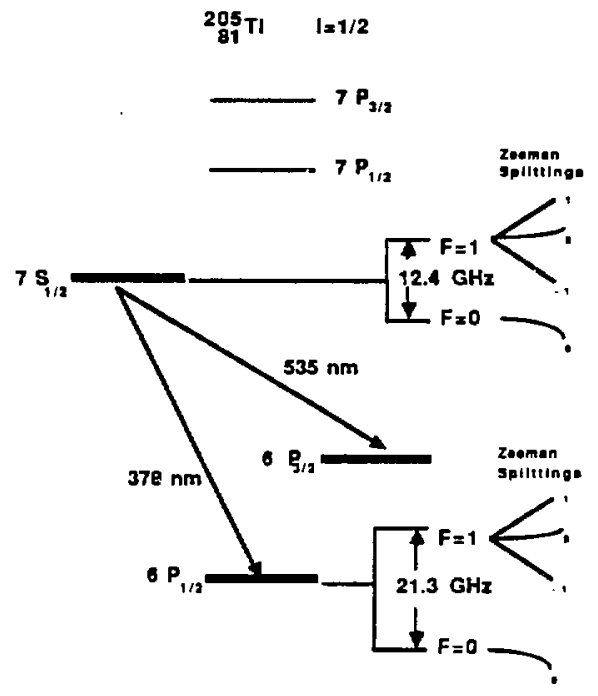

Figure III.1 
We apply an average magnetic field $\mathrm{B}_{0}=.28$ gauss which splits the $\mathrm{F}=1$ hyperfine level of the ground state into 3 Zeeman sublevels $m_{F}=-1,0,+1$, with energy splittings of

$$
\Delta \mathrm{E}=\mathrm{gF}_{\mathrm{F}} \mu_{0} \mathrm{~B}_{0}=130 \mathrm{kHz}
$$

If the thallium $6 \mathrm{P}_{1 / 2}$ ground state has a non-zero EDM $\mathrm{d}_{\text {atom }}$, it must point along $\langle\vec{F}\rangle$. since the atom possesses no other well-defined direction. As shown below in Figure III. 2 , the $m_{F}=-1$ and the $m_{F}=+1$ sublevels will exhibit opposite energy shifts in an applied, external electric field.

\section{The Experiment}

\section{(How do we measure d atom?)}

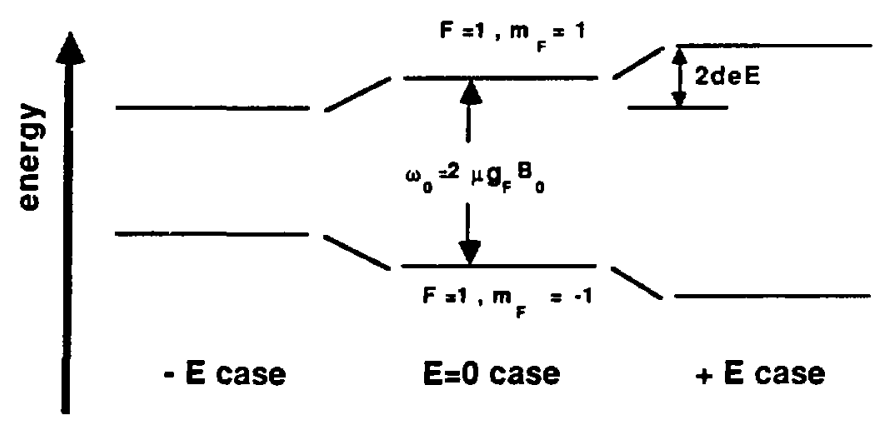

Figure III.2

Two counterpropagating, vertical thallium beams are produced by thermal effusion from ovens. In a vertical beam, atoms with different velocities have the same trajectories because the acceleration due to gravity is along the beam. The atoms are optically pumped with $378 \mathrm{~nm}$ light $\left(6 \mathrm{P}_{1 / 2} \rightarrow 7 \mathrm{~S}_{1 / 2}\right)$ into the $6 \mathrm{P}_{1 / 2}\left(\mathrm{~F}=1, \mathrm{~m}_{\mathrm{F}}=0\right)$ ground state. A pair of of loops, with a relative phase shift of $\pm \pi / 4$ or $\pm 3 \pi / 4$ radians, 
induces transitions between the $\mathrm{F}=1$ magnetic sublevels. If the atom has a non-zero EDM, there will be an energy shift that reverses with the electric field direction. The shift is detected by measuring the $535 \mathrm{~nm}$ fluorescence $\left(7 \mathrm{~S}_{1 / 2} \rightarrow 6 \mathrm{P}_{3 / 2}\right)$ generated by optical pumping at the detector.

The experiment is designed to minimize the foreseeable sources of noise and systematics. The direction of the atomic beam, the phase difference between the two if loops, and the electric field are periodically reversed in order to separate an EDM from noise and systematics. In particular, the two opposing beams serve to cancel a $\mathrm{v} \times \mathrm{KE}$ effect, a systematic arising through the motional magnetic field that an atom experierices when travelling through a strong electric field. The chops not only isolate the EDM from systematics, but also remove noise that is characterized by frequencies lower than the chopping rate. For example, the faster one chops the electric field, the broader the spectrum of magnetic noise that is reduced.

We now present a schematic of the apparatus in Figure III.3 and present a simplified picture of the evolution of an atom's wavefunction in the upgoing beam in order to better describe the function of each component. For simplicity, we assume that the if power is perfectly adjusted to the velocity of the atom.

The upgoing atom leaves the lower oven in an incoherent superposition of states. The laser then acts as a polarizer and drives the atom into the $\mathrm{m}_{\mathrm{F}}=0$ srate:

$$
\psi_{1}=\left(\begin{array}{l}
0 \\
1 \\
0
\end{array}\right)
$$

The first of field then causes a transition to the $m_{F}=1$ and $m_{F}=-1$ sublevels:

$$
\Psi_{2}=\frac{1}{\sqrt{2}}\left(\begin{array}{c}
1 \\
0 \\
-1
\end{array}\right)
$$


If there is a non-zero electron EDM, these two states will acquire phase-shifts $\mathrm{e}^{\mathrm{iu}}$ and $\mathrm{e}^{-\mathrm{i} u}$ respectively in the electric field, where $u$ is the phase-shift resulting from the EDM-electric field interaction energy:

$$
\psi_{3}=\frac{1}{\sqrt{2}}\left(\begin{array}{c}
\mathrm{e}^{\mathrm{iu}} \\
0 \\
-\mathrm{e}^{-\mathrm{i} u}
\end{array}\right)
$$

If the second If loop is $\pi / 4$ radians out of phase with the first loop, then the atom exits the loop in a state

$$
\Psi_{4}=\frac{-1}{2 \sqrt{2}}\left(\begin{array}{c}
(1+i)(1-u) \\
2(1+u) \\
(-1+i)(1-u)
\end{array}\right)
$$

The fluorescent detector analyzes this state and gives a signal from which $\mathrm{u}$, and hence the electric dipole moment, can be inferred.

$$
S=\frac{1}{2}(1-2 u)
$$

We will discuss each aspect of the experiment in detail in the sections that follow. 


\section{The Apparatus}

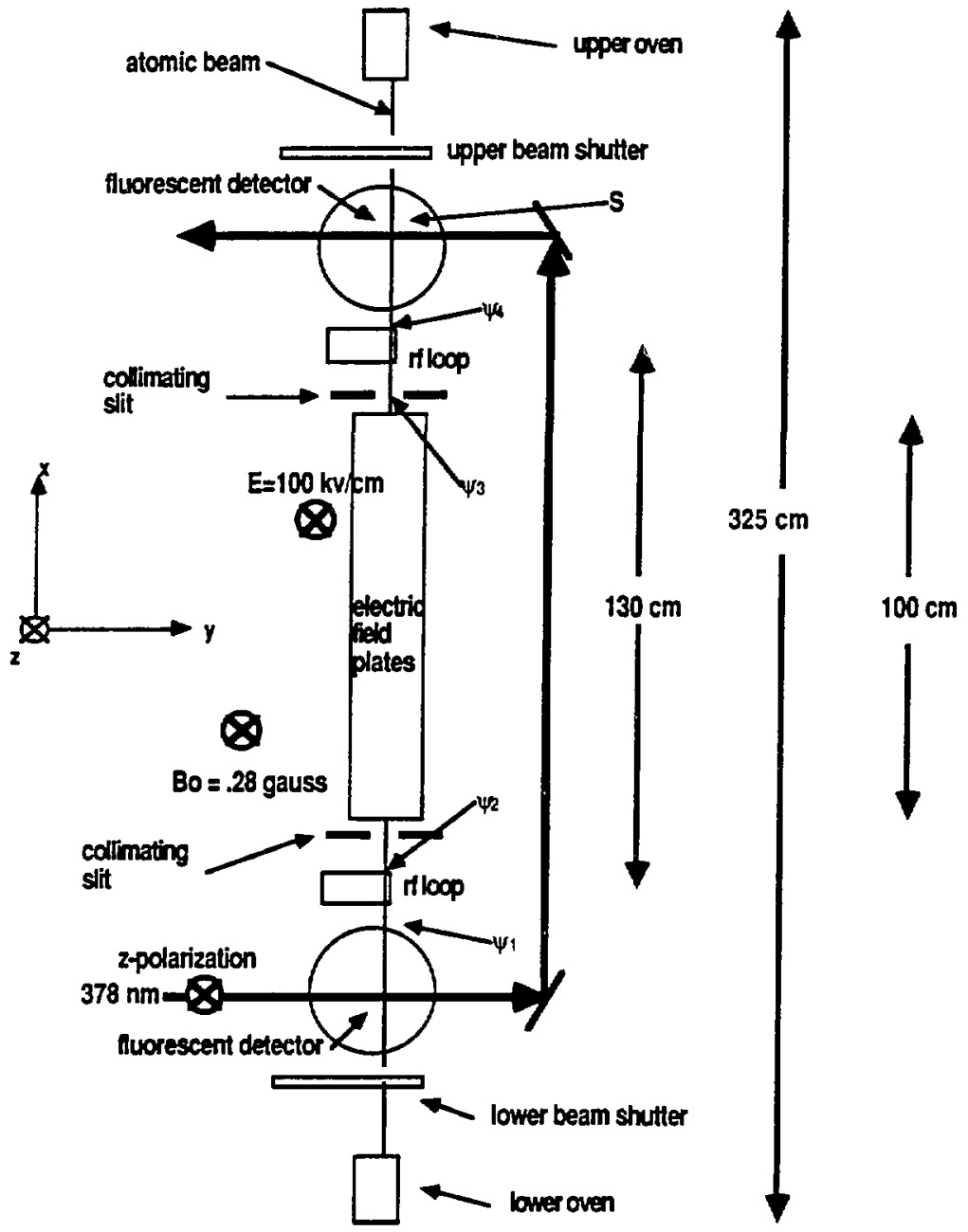

Figure III.3 


\section{B. The Atomic Beam}

\section{The Vacuum Can and Pressures}

The experiment is enclosed in a vertical aluminum (non-magnetic) vacuum can which is divided into a single main chamber, two buffer chambers, and two oven chambers. The majority of the flanges are sealed to the can with $1 / 8$ " viton o-rings. Four BayardAlper type ion gauges measure the pressure in each of the buffer and oven chambers; the main chamber pressure must be inferred from the buffer chamber pressures. Pumping is provided by two 6" Varian diffusion pumps and two 4" Varian diffusion pumps, all four of which use SANTOVAC 5 oil and are backed by two $12 \mathrm{cfm}$ Welsh mechanical pumps. The mechanical pumps maintain a foreline pressure of better than 60 microns. The aluminum manifolds that connect the diffusion pumps to the vacuum chamber throttle each pump's pumping speed down to about 100 liters/second.

Additional pumping is provided by a brass, cylindrical nitrogen cold trap, which measures $10.2 \mathrm{~cm}$ in diameter and $79 \mathrm{~cm}$ in length (liquid capacity is 6.5 liters) and is vertically situated in the main chamber. The trap must be refilled once every 12 hours in order to maintain an acceptable pressure. Under normal operating conditions, the pressure in the buffer chamber is about $7 \times 10^{-7}$ torr, when the trap is full.

The atomic beam should experience an exponential attenuation of the form $A=e^{-L \lambda}$, where $L=220 \mathrm{~cm}$ (the distance from an oven to the opposite fluorescent detector), $\lambda=\frac{1}{\mathrm{n} \sigma}$ is the mean free path of a thallium atom in the main chamber, $\mathrm{n}$ is the number derisity of the scattering molecules, and $\sigma$ is the average individual cross-section for collisions of a thallium atom with background gas. If we use the values $\mathbf{n}=3 \times 10^{10}$ $/ \mathrm{cm}^{3}$, which corresponds to $7 \times 10^{-7}$ torr, and the cross-section of a typical molecule $\sigma=$ $1 \times 10^{-14} \mathrm{~cm}^{2}$, we find a theoretical mean free path of $\lambda=3000 \mathrm{~cm}$. However, we made 
two separate measurements that indicate $\lambda \ll 3000 \mathrm{~cm}$.

In the first, we recorded the fluorescent intensity of the $7 \mathrm{~S}_{1 / 2} \rightarrow 6 \mathrm{P}_{3 / 2}$ transition as a function of pressure. Pressures between $1 \times 10^{-6}$ torr and $6 \times 10^{-6}$ torr were achieved by throttling one of the four diffusion pumps. Figure III.4 shows that the data are in complete disagreement with $\lambda=3000 \mathrm{~cm}$ and suggest a mean free path $\lambda<300 \mathrm{~cm}$.

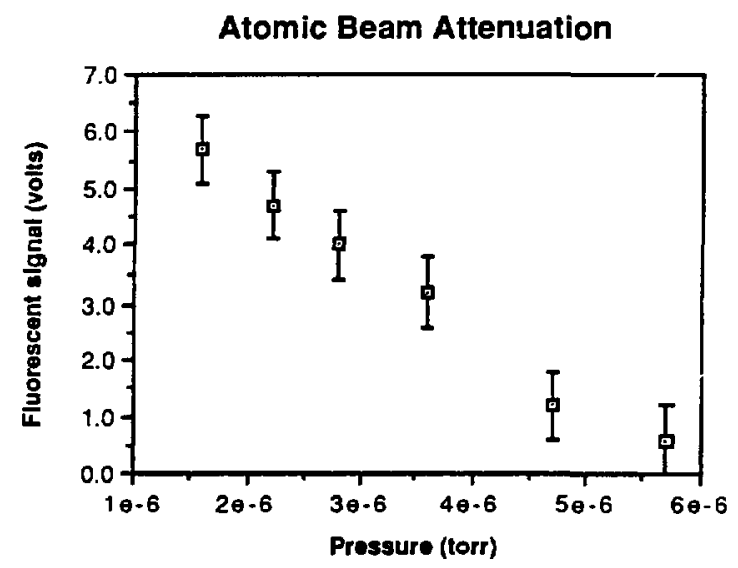

Figure III.4

The graph appears to be almost linear; there are no obvious signs of leveling off at low pressures. 
In the second test, we measured the ratio of the fluorescent signals at the far and near detectors. During this test, a temporary slit, with dimensions $.2 \mathrm{~cm} \mathrm{x} 1 \mathrm{~cm}$, was located at the fluorescent detector nearest the oven (near detector). Before recording the ratio, we fully optimized the oven, slits, and laser beam positions for a maximura signal at the far detector. We found the ratio $\frac{S_{\text {near }}}{S_{\text {far }}}=40$, whereas the expected ratio from solid angle considerations in the absence of scattering is

$$
\left(\frac{l_{\text {far }}}{I_{\text {near }}}\right)^{2}\left(\frac{A_{\text {near }}}{A_{\text {far }}}\right)=\left(\frac{210 \mathrm{~cm}}{80 \mathrm{~cm}}\right)^{2}\left(\frac{.2 \mathrm{~cm} \times 1 \mathrm{~cm}}{.1 \mathrm{~cm} \times 1 \mathrm{~cm}}\right)=14 .
$$

where the A's denote the are is of the near and far slits. If we assume that the pressure readings in the oven and buffer chambers are accurate and that the discrepancy lies in the main chamber pressure, we are left with a mean free path $\lambda \approx 190 \mathrm{~cm}$ which corresponds to a main chamber pressure of $3 \times 10^{-5}$ torr. This is unlikely. Other possible explanations include scattering of $\mathrm{Tl}$ atoms off of the slits and collisions with extremely large organic molecules.

\section{The Ovens}

Coiled tantalum wires, insulated by ceramic spacers, heat the experiment's two conventional, stainless steel ovens to temperatures between 900 and 1100 degrees Kelvin. Each oven's temperature is controlled by two separate 20 ampere - $60 \mathrm{~Hz}$ variacs, one for the reservoir and the other for the slit. It is important to keep the slits at least $20^{\circ} \mathrm{K}$ $u$ srmer than the reservoirs in order to maintain an even, consistent atomic beam. Roughly 900 watts of power is required to maintain a temperature of $1100^{\circ} \mathrm{K}$. Two chromel-alumel (Type-K) thermocouples monitor the slit and reservoir temperatures of each oven. Their 
signals are fed into an OMEGA Model 650 Temperature Indicator unit that converts volts to degrees Centigrade. The two ovens are nearly identical except that the lower oven's slit is situated on its top in order in deliver an upgoing beam, while the upper oven's is situated on its underside.
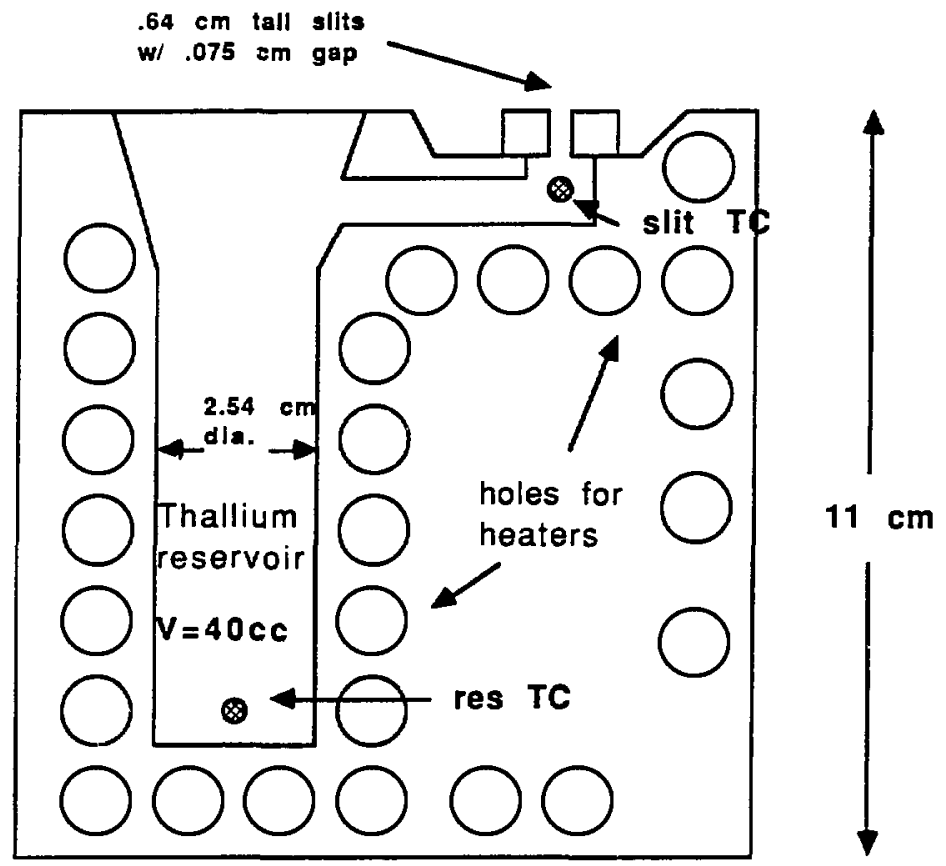

$10.1 \mathrm{~cm}$

A cross-sectional drawing of the lower oven

Figure III.5 


\section{The Atomic Flux and Velocity Distribution}

\section{The Flux}

The following table gives the pressure, the density $\mathrm{n}$, the mean free path $\lambda$, and the average velocity of thallium atoms inside an oven $\overline{\mathrm{v}_{0}}$ as a function of temperature. The average velocity in the oven is given by conventional gas kinetics

$$
\bar{v}_{0}=\frac{2}{\sqrt{\pi}} \alpha_{0}=\frac{2}{\sqrt{\pi}} \sqrt{\frac{2 \mathrm{kBT}_{\mathrm{B}}}{\mathrm{m}}}
$$

where $\alpha_{0}$ is the "most probable" velocity in the oven. The mean free path $\lambda \approx \frac{1}{n \sigma}$ is calculated using a model cross-section of $10^{-14} \mathrm{~cm}^{2}$.

$\begin{array}{lllll}\text { temperature }\left({ }^{\circ} \mathrm{K}\right) & \text { pressure }(\mathrm{tor}) & \frac{\mathrm{n}\left(\mathrm{cm}^{-3}\right)}{1.7 \times 10^{14}} & \frac{\lambda(\mathrm{cm})}{.58} & \overline{\mathrm{v}_{\mathrm{o}}}(\mathrm{cm} / \mathrm{sec}) \\ 900 & 1.6 \times 10^{-2} & 1.7 \times 10^{4} & 3.0 \times 10^{4} \\ 1000 & 1.6 \times 10^{-1} & 1.5 \times 10^{15} & .067 & 3.2 \times 10^{4} \\ 1100 & 9.8 \times 10^{-1} & 8.6 \times 10^{15} & .012 & 3.4 \times 10^{4} \\ 1200 & 4.5 & 3.6 \times 10^{16} & .003 & 3.5 \times 10^{4}\end{array}$

At these temperatures, there are basically two flow regimes: effusive, where $\lambda>w$ (slit width) and the atoms do not interact, and visccus, where the flow might resemble water spraying from a nozzle. Because the slit width is $.075 \mathrm{~cm}$, the highest temperature at which an oven can operate, while still remaining in the effusive regime, is about $1000^{\circ} \mathrm{K}$.

We now estimate the expected useful flux of atoms reaching the far detector $F_{\mathrm{d}}$. For an effusive beam, the total flux leaving the oven is $F_{0}=\frac{1}{4} A n \bar{v}$, where $A=.075 \mathrm{~cm} \times .5$ $\mathrm{cm}=.0375 \mathrm{~cm}^{2}$ is the area of the slit. The flux of atoms traversing the apparatus is reduced by two collimating slits, located at either end of the electric field plate assembly, which define the beam's transverse dimensions of $1 \mathrm{~cm} \times 1 \mathrm{~cm}$. The solid angle subtended 
by the farthest slit from the oven is $\mathrm{d} \Omega_{\mathrm{eff}} / 4 \pi=1 / \pi \times \mathrm{A} / \mathrm{L}_{\mathrm{far}}{ }^{2}=.1 \times 1 /\left(210^{2} \times \pi\right)=8$ $\times 10^{-7}$, and, using the table information for $\mathrm{T}=1000^{\circ} \mathrm{K}$, we expect a flux at the far detector of roughly $F_{d}=4 \times 10^{11}$ atoms/second. In addition, scattering in the main chamber further reduces the flux by a factor of $A \approx \frac{40}{14} \approx 3$. Finally, we include factors of .7 for the $\mathrm{Tl}^{205}$ abundance and $3 / 10$ for optical pumping efficiency. This gives an expected flux of $F_{d}=2 \times 10^{10}$ at $\mathrm{T}=1000^{\circ} \mathrm{K}$. Figure III.6 displays our measurements of the flux of atoms at the far detector versus oven reservoir temperature.

\section{Measured Atomic Flux vs Temperature}

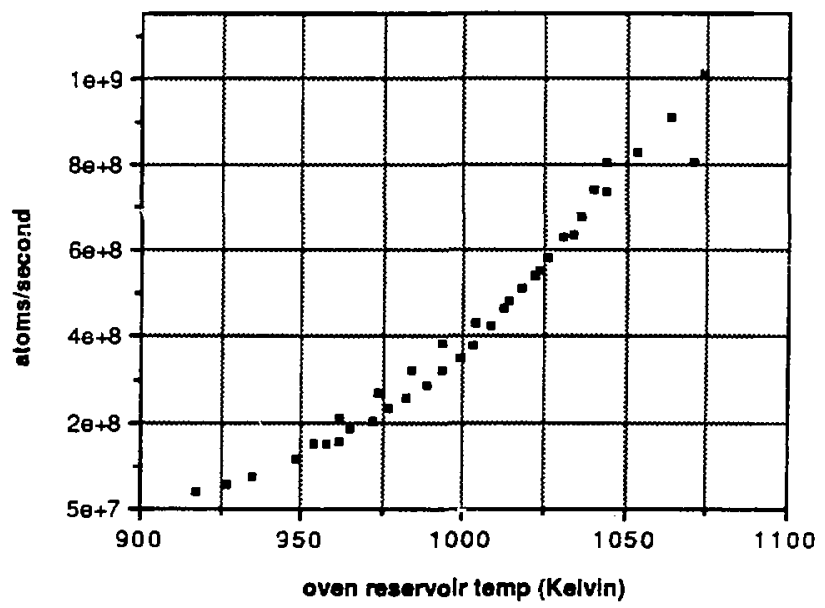

Figure III.6

Having estimated a detector efficiency of $4 \%$ (see § III.D), it is not difficult to deduce the atomic flux from raw signals. For example, when the ovens are at a temperature of $\mathrm{T}_{\text {reservoir }}=950^{\circ} \mathrm{K}$ and $\mathrm{T}_{\text {slit }}=990^{\circ} \mathrm{K}$, we measured a $125 \mathrm{nA}$ current at the phototube 
anode. With 600 volts applied to the phototube, it has a gain $=10^{5}$. Therefore, the cathode current is $I_{\text {Eathode }}=\left(125 \times 10^{-9}\right)\left(10^{-5}\right)=1.3 \times 10^{-12}$ amps and the number of photoelectrons is simply $\mathrm{F}_{\mathrm{pe}}=\mathrm{I}_{\text {catiode }} /\left(1.6 \times 10^{-19}\right.$ coulomb/electron $)=7.8 \times 10^{6}$ photoelectrons/second. Dividing by the overall detector efficiency, we obtain an atomic flux $F_{d}=2 \times 10^{8}$ atoms/second.

We immediately see a discrepancy of a factor of 50 between the expected and measured fluxes. This could be due to an error in the gain of the phototube. The mild increase of the flux with temperature is due to the exponential increase of the vapor pressure $p(T)$ as a function of temperarure $T$.

\section{The Velocity Distribution}

The beam has a $v^{3}$ Maxwell-Boltzmann velocity distribution where the flux with velocity $v$ is $F_{0}(v)=2 \frac{F_{0}}{\alpha_{0}^{4}} v^{3} e^{\frac{-v^{2}}{\alpha_{0}^{2}}}$. We verified the velocity distribution both by pulsing the atomic beam and by optically selecting particular velocity components via the Doppler effect. We will not discuss these measurements in detail, but we present a calculation of $F_{0}(v)$ with $\alpha_{0}=3.0 \times 10^{4} \mathrm{~cm} / \mathrm{sec}$ and a measured distribution with $\mathrm{T} \approx 1000^{\circ} \mathrm{K}$

\section{Velocity Distribution of Atomic Beam}

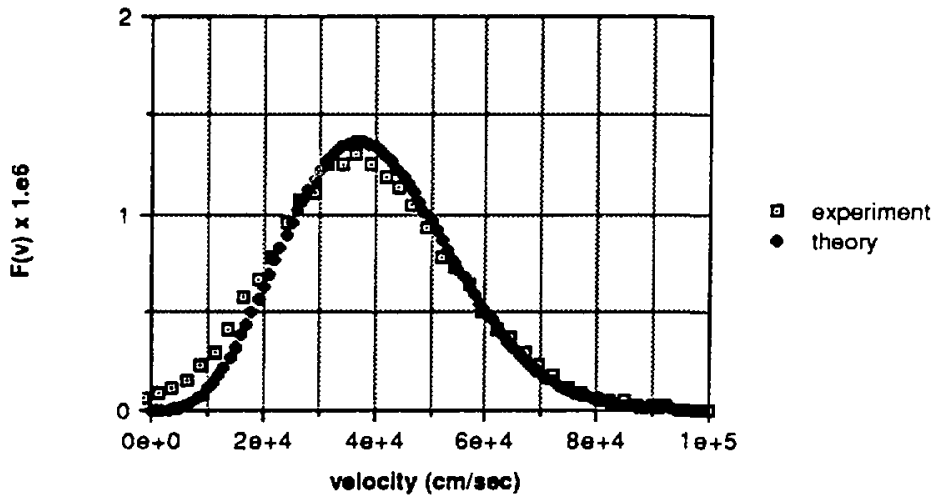

Figure III.7 
In order to compare the two distributions and because no absolute velocity measurement was available, we matched the peak velocities and amplitudes of the distributions. Experiment and theory agree quite favorably, although there might be an abundance of slow atoms in the measured distribution.

The peak of the beam's velocity distribution is not at $v=\alpha_{0}$ - rather it has the slightly higher value $\alpha=\sqrt{\frac{3}{2}} \alpha_{0}$. Similarly, the average velocity of the beam can be related to tha: in the oven by

$$
\bar{v}=\frac{3}{4} \sqrt{\pi} \alpha_{0}=\frac{3}{8} \pi \overline{v_{0}}
$$

Throughout this paper, when dealing with quantities averaged over the velocity distribution, we will always use the average velocity of the beam $\bar{v} \approx 4 \times 10^{4} \mathrm{~cm} / \mathrm{sec}$.

\section{The Spatial Distribution}

If thit oven slits were infinitely thin, the atomic beam would have $a \cos \theta$ spatial distribution. The tall slits which we use $\left(l_{\text {slit }}=.64 \mathrm{~cm}\right.$ long,$w_{\text {slit }}=.075 \mathrm{~cm}$ wide $)$ serve both to reduce wasted atoms leaving the oven at oblique angles and also to peak the spatial distribution in the forward direction. The former effect is a characteristic feature of effusive beams in which the total oven output is revised to $F_{0}=\frac{1}{4 \kappa} n A \bar{v}$ with $\frac{1}{\kappa}=\frac{w_{s l i t}}{l_{s l i t}} \log \frac{l_{s l i t}}{w_{\text {slit }}}=.2535$. The latter effect, which is analogous to attaching a nozzle to a water hose, is absent in a purely effusive beam. We found that by changing $\mathrm{l}_{\text {slit }}$ from $.32 \mathrm{~cm}$ to $.64 \mathrm{~cm}$, we increased our forward flux by a factor of eight. This increase might be a result of decreasing the pumping conductance of the slit, thereby increasing the $T I$ vapor pressure inside oven.

\section{Thallium Load Exhaustion TIme}

35 Norman Ramsey,Molecular Beams(Oxford University Press, Clarendon, 1956), p. 14. 
We found that it takes approximately $10-15$ days of continuous running at $\mathrm{T}_{\text {res }}=$ $1100^{\circ} \mathrm{K}$ for an oven to fully exhaust its load. This time agrees reasonably well with the theoretical prediction of 23 days: for $\mathrm{T}_{\mathrm{res}}=1100^{\circ} \mathrm{K}$,

$$
\mathrm{F}_{0}=\frac{1}{4 \kappa} \mathrm{nA} \overline{\mathrm{v}_{\mathrm{o}}}=7 \times 10^{17} / \mathrm{sec}
$$

The volume of the thallium reservoir is $40 \mathrm{cc}$ and, therefore, contains

$$
\mathrm{N}=\frac{\left(40 \mathrm{~cm}^{3}\right)\left(12 \mathrm{gram} / \mathrm{cm}^{3}\right)}{(205)\left(1.7 \times 10^{-24} \mathrm{gram}\right)} \approx 1.4 \times 10^{24}
$$

atoms. Dividing the two gives $\mathrm{N} / \mathrm{F}_{0}=23$ days. When the oven is loaded, gaps remain between individual pieces of thallium metal - this could easily explain a factor of two discrepancy in exhaustion time. 


\section{Polarizing and Analyzing with Optical Pumping}

\section{The Optical Pumping Technique}

A major advantage of our experiment over past experiments is the use of lasers for polarizing and analyzing the atomic wavefunctions via optical pumping. In the past, experimenters employed the Rabi - Stern - Gerlach technique of atomic state selection by passing an atom with non-zero magnetic dipole moment through a non-uniform magnetic field. Depending on its state or magnetic sublevel, an atom would travel along different trajectories, thus enabling experimenters to include or exclude it in the useable atomic beam.

This technique has many drawbacks. Not only are the large polarizing magnets space-consuming, but they also produce substantial magnetic fields in an experiment in which a well defined, stable field is of paramount importance. In addition, atoms with different velocities would naturally experience different deflections thereby complicating the state selection process. State selection by optical pumping is both more effective and easier to implement.

With the advent of lasers, the atomic optical pumping technique, first pioneered by Alfred Kastler in the late 1950's, has become a standard laboratory tool. Based entirely on the conservation of angular momentum, it allows complete control of an atom's state through the choice of the polarization of the laser light which pumps a certain transition.

The energy levels of thallium in zero external field are depicted in Appendix D. We tune the lasers to the $\mathrm{E}_{1}$ allowed $6 \mathrm{P}_{1 / 2}(\mathrm{~F}=1) \rightarrow 7 \mathrm{~S}_{1 / 2}(\mathrm{~F}=1)$ transition and measure the $7 \mathrm{~S}_{1 / 2} \rightarrow 6 \mathrm{P}_{3 / 2}$ fluorescence at $\lambda=535 \mathrm{~nm}$. On account of the relatively narrow power broadened transition linewidth $\Delta v_{\text {eff }}=250 \mathrm{MHz}$, and the large hyperfine splittings of these states $\left(21.3 \mathrm{GHz}\right.$ for the $6 \mathrm{P}_{1 / 2}$ and $12.4 \mathrm{GHz}$ for the $\left.7 \mathrm{~S}_{1 / 2}\right)$, no other hyperfine 
levels are pumped. The laser does, however, pump all Zeeman components since they lie within $.13 \mathrm{MHz}$ of each other when $\mathrm{B}_{0}=.28$ gauss. The decay rate for $7 \mathrm{~S}_{1 / 2} \rightarrow 6 \mathrm{P}_{3 / 2}$ is comparable to the rate for $7 \mathrm{~S}_{1 / 2} \rightarrow 6 \mathrm{P}_{1 / 2}$, but because the $6 \mathrm{P}_{3 / 2}$ state is metastable with a lifetime $(\tau \approx .3 \mathrm{sec})$ longer than the transit time of the atoms through the experiment, any atoms in this level are effectively absent from the experiment.

We now discuss in detail the method by which we polarize the atoms into our desired initial state of $6 \mathrm{P}_{1 / 2}\left(\mathrm{~F}=1, \mathrm{~m}_{\mathrm{F}}=0\right)$, delaying until the next two sections our discussion of laser power, polarization, and other characteristics. Upon leaving the oven and before interacting with the laser, an atom is in an incoherent superposition of the $F=0$ and $F=1$ $6 \mathrm{P}_{1 / 2}$ ground state hyperfine levels. Although the $\mathrm{F}=1$ state lies $21.3 \mathrm{GHz}$ above the $\mathrm{F}=0$ level, the probabilities for the two states are effectively equal because the Boltzmann factor $\mathrm{e}^{\frac{-\Delta \mathrm{E}}{\mathrm{k}_{\mathrm{a}} \mathrm{T}}} \approx 1$ for an atom at temperature $\mathrm{T}=1000^{\circ} \mathrm{K}$

For light that is linearly polarized in the $z$-direction and a quantizing magnetic field $\mathrm{B}_{0}$ pointing in the $\mathrm{z}$-direction, the selection rule for an El optical transition is $\Delta \mathrm{m}_{\mathrm{F}}=0$. After cycling through the $6 \mathrm{P}_{1 / 2}(\mathrm{~F}=1)$ and $7 \mathrm{~S}_{1 / 2}(\mathrm{~F}=1)$ levels via stimulated absorption and spontaneous emission, an atom is left in the $6 \mathrm{P}_{1 / 2}\left(\mathrm{~F}=1, \mathrm{~m}_{\mathrm{F}}=0\right)$ state since ransitio.s out of this state are forbidden - the Clebsch-Gordan coefficient connecting this level to the $7 \mathrm{~S}_{1 / 2}\left(\mathrm{~F}=1, \mathrm{mF}_{\mathrm{F}}=0\right)$ state vanishes: $\langle 1,0 ; 1,0 \mid 1,0\rangle=0$. Figure III.8 depicts the various processes involved in the chosen optical pumping configuration. 
Optical Pumping with z-Polarization

Result: Polarization in $F=1, m_{F}=0$ State
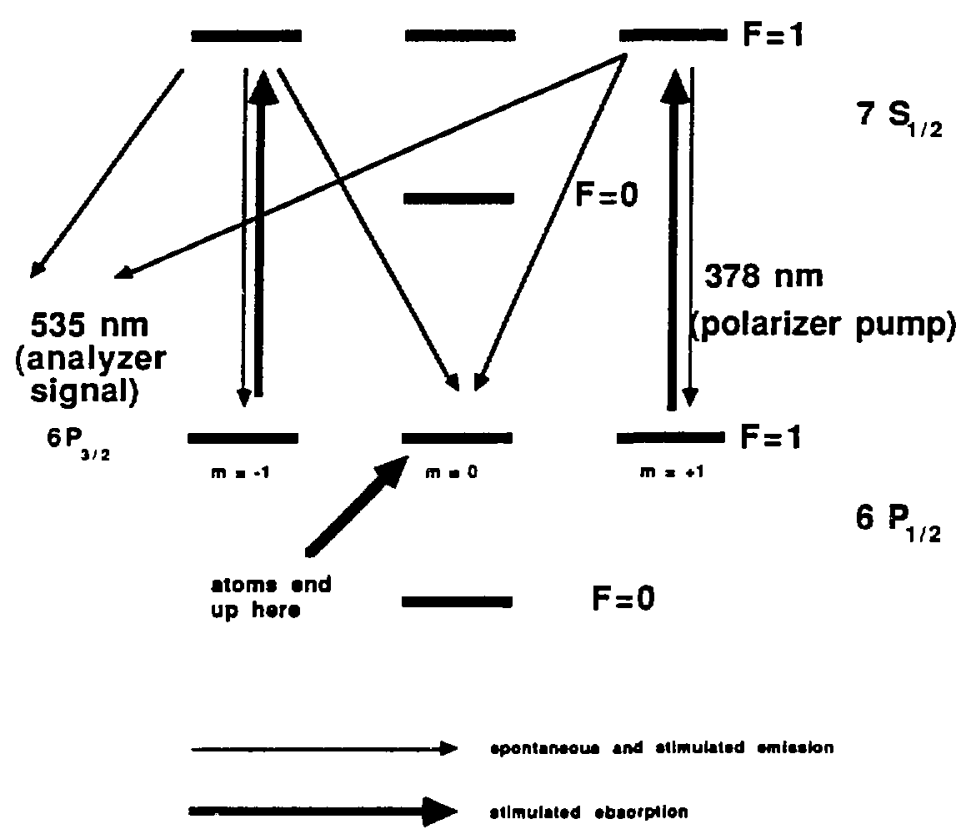

Figure III.8

Optical pumping is equally effective as an atomic state analyzer. The total probability of an atom emitting a $535 \mathrm{~nm}$ photen is equal to the sum of the probabilities for the $\mathrm{mF}=$ -1 and the $m_{F}=+1$ states. Thus, if an atom's $6 \mathrm{P}_{1 / 2}(F=1)$ wavefunction is

$$
\psi=\left(\begin{array}{l}
a \\
b \\
c
\end{array}\right)
$$

then the observed fluorescent signal is 


$$
\mathrm{S}_{535 \mathrm{~nm}}=|\mathrm{a}|^{2}+|\mathrm{c}|^{2}
$$

\section{Rate Equations and Optical Linewidth}

In this section we discuss the rate equations for optical pumping and demonstrate that a few milliwatts of $378 \mathrm{~nm}$ light is just enough power to fully polarize and analyze the atoms. We also examine the various transition linewidths involved and present an experimental optical pumping curve.

The spontaneous decay rate for $7 \mathrm{~S}_{1 / 2} \rightarrow 6 \mathrm{P}_{1 / 2}$ is

$$
\mathrm{A}_{0}\left(7 \frac{\mathrm{S}}{2} \rightarrow 6 \mathrm{P}_{\frac{1}{2}}\right)=7 \times 10^{7} \mathrm{sec}^{-1}
$$

Because $\Delta v_{\text {laser }}=1 \mathrm{MHz} \ll \Delta v_{\text {atom }}$, it can be shown that the stimulated absorption rate is given by 36

$$
\Gamma=\frac{2 \pi \alpha}{\bar{\hbar}}|\langle\bar{\varepsilon} \cdot \bar{r}\rangle|^{2} I_{378 \mathrm{sm}} g(\omega)
$$

where the Lorentzian lineshape function is

$$
g(\omega)=\frac{2 A_{0}}{\left(\omega-\omega_{0}\right)^{2}+A_{0}^{2}}
$$

and the square of the matrix element in our case is

$$
|\langle\bar{\varepsilon} \cdot \bar{I}\rangle|^{2}=\left|\left\langle\mathrm{S}_{1 / 2}, \mathrm{~F}=1, \mathrm{~m}_{\mathrm{F}}=1|\mathrm{z}| 6 \mathrm{P}_{1 / 2}, \mathrm{~F}=1, \mathrm{~m}_{\mathrm{F}}=1\right\rangle\right|^{2} .
$$

From (III.C.4), we immediately see that the natural, or homogeneous linewidth is $\Delta v_{\text {natural }}=\frac{A_{0}}{\pi}=20 \mathrm{MHz}$. We shall see that the excess applied laser power broadens this width considerably.

The rate equations describe the three processes of stimulated absorption, stimulated 
emission, and spontaneous emission between the $6 \mathrm{P}_{1 / 2}(\mathrm{~F}=1, \mathrm{~F}=0), 6 \mathrm{P}_{3 / 2}(\mathrm{~F}=2, \mathrm{~F}=1)$, and $7 S_{1 / 2}(F=1)$ states. Before writing down these differential equations, we first decompose the five relevant hyperfine states into their corresponding electronic and nuclear angular momentum component wavefunctions:

$$
\begin{aligned}
17 S_{1 / 2}, F=1, m_{F}=1>= & R_{7 s} Y_{00} \alpha_{e} \alpha_{N} \\
\mid 6 P_{1 / 2}, F=1, m_{F}=1>= & \sqrt{\frac{2}{3}} R_{6 P} Y_{11} \beta_{e} \alpha_{N}-\sqrt{\frac{1}{3}} R_{6 P} Y_{10} \alpha_{e} \alpha_{N} \\
16 P_{1 / 2}, F=1, m_{F}=0>= & -\sqrt{\frac{1}{6}} R_{6 P} Y_{10} \alpha_{e} \beta_{N}+\sqrt{\frac{1}{3}} R_{6 P} Y_{11} \beta_{e} \beta_{N} \\
& +\sqrt{\frac{1}{6}} R_{6 P} Y_{10} \beta_{e} \alpha_{N}-\sqrt{\frac{1}{3}} R_{6 P} Y_{1-1} \alpha_{e} \alpha_{N}
\end{aligned}
$$

The analysis is the same for the other relevant states. Here, the Y's denote the standard spherical harmonics, the R's are the atomic radial wavefunctions, and $\alpha$ and $\beta$ represent spin up and spin down respectively from the electron and nucleus. We then calculate the allowed transition rates in terms of the appropriate Clebsch-Gordan coefficients and a reduced matrix element $\left\langle R_{6 P}\|r\| R_{78}\right\rangle$.

However, such a detailed treatment is unnecessary if all we want is a basic idea of the optical pumping lineshapes and laser power requirements. We will simply assign a rate of $A_{0} / 3$ to each allowed spontaneous decay channel. Our stimulated absorption matrix element is $\sqrt{\frac{1}{3}}\left\langle R_{6 \mathrm{P}} \mathrm{Y}_{10}|\mathrm{z}| \mathrm{R}_{7 \mathrm{~S}} \mathrm{Y}_{00}\right\rangle$, which can be calculated to yield to value of $4.2 \times$ $10^{-9} \mathrm{~cm}$. 


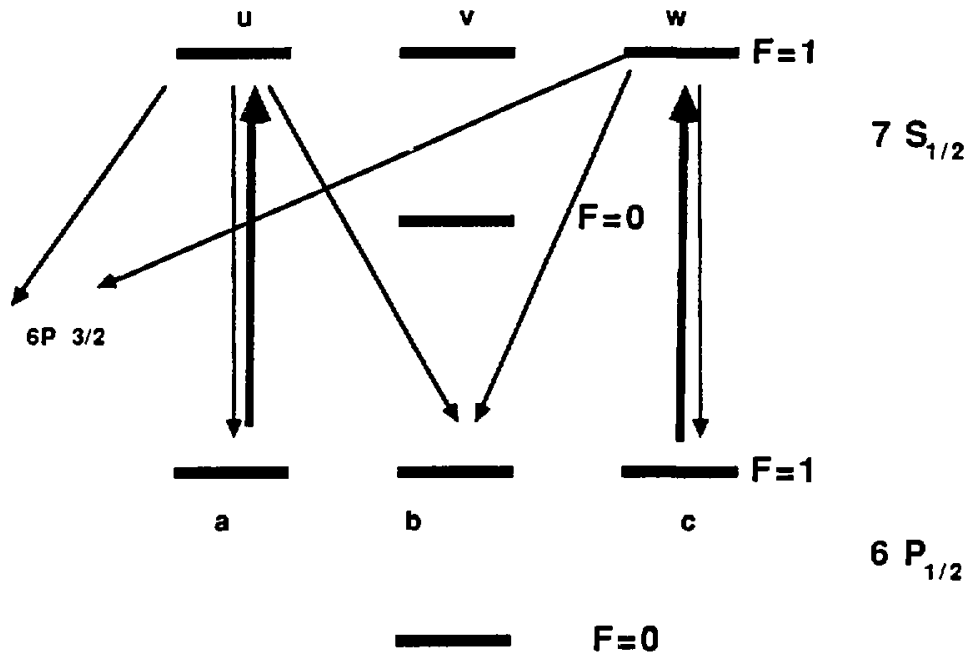

Figure III.9

In Figure III.9, the variables $a, b, c, u, v$, and $w$ denote the populations (probabilities) of :se various states. Inicially, the atom is in the $6 \mathrm{P}_{1 / 2}$ ground state and, accordingly, we have the initial conditions $a(0)=1 / 3, b(0)=1 / 3$, and $c(0)=1 / 3$. The $7 S_{1 / 2}$ excited state is empty and $u(0)=: \because(0)=w(0)=0$. For $z$-polarized light, the rate equations are

$$
\begin{aligned}
& \frac{d a}{d t}=\Gamma(u-a)+\frac{A_{0}}{3} u \\
& \frac{d b}{d t}=\Gamma\left(\frac{u}{2}+\frac{w}{2}-b\right)+\frac{A_{0}}{3} u+\frac{A_{0}}{3} w \\
& \frac{d c}{d t}=\Gamma(w-c)+\frac{A_{0}}{3} w \\
& \frac{d u}{d t}=\Gamma(a-u)-2 A_{0} \\
& \frac{d w}{d t}=\Gamma(c-w)-2 A_{0}
\end{aligned}
$$

It is interesting to note that the final population of the $6 \mathrm{P}_{1 / 2}\left(\mathrm{~F}=1, \mathrm{~m}_{\mathrm{F}}=0\right)$ state is 
approximately $b(f)=1 / 4 \times 7 / 5=7 / 20$. In other words, only about $30 \%$ of the atoms entering the polarizer exit in the proper polarized state.

These equations were integrated numerically and the optical pumping lineshapes for $I_{0}=30 \mathrm{~mW} / \mathrm{cm}^{2}$ (see next section) agreed quite well with observations. Both the calculation and observations give a saturation broadened linewidth of $\Delta v_{\text {sat }} \approx 200 \mathrm{MHz}$ and a pumping "dip width" of $\Delta \mathrm{v}_{\text {dip }}=80 \mathrm{MHz}$. We found that optical saturation is adequate down to a minimum acceptable dip width of $20 \mathrm{MHz}$. Figure III.10 displays measured optical pumping curves, while Figure III.11 represents the solution to the rate equations. The agreement is exceptional. The background in the observed curves is a result of scartered atoms interacti.1g with the laser beam (see § III.K.5).

\section{Typical Optical Pumping Curves}

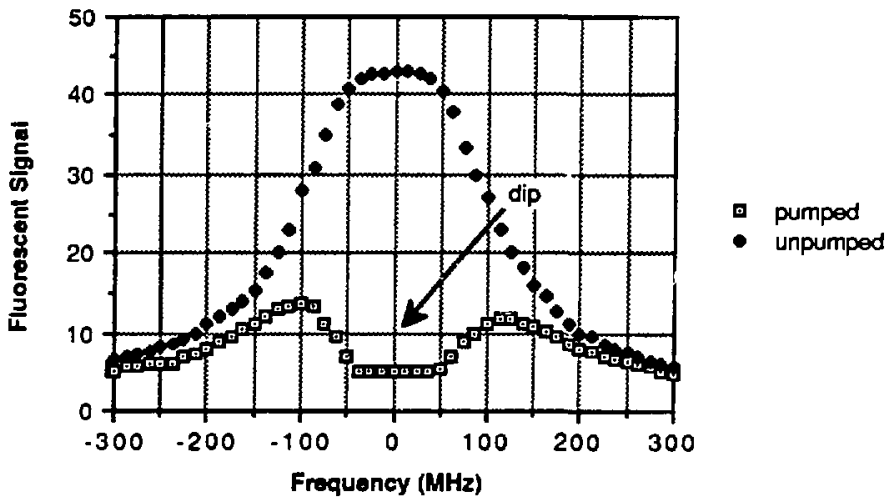

Figure III.10 


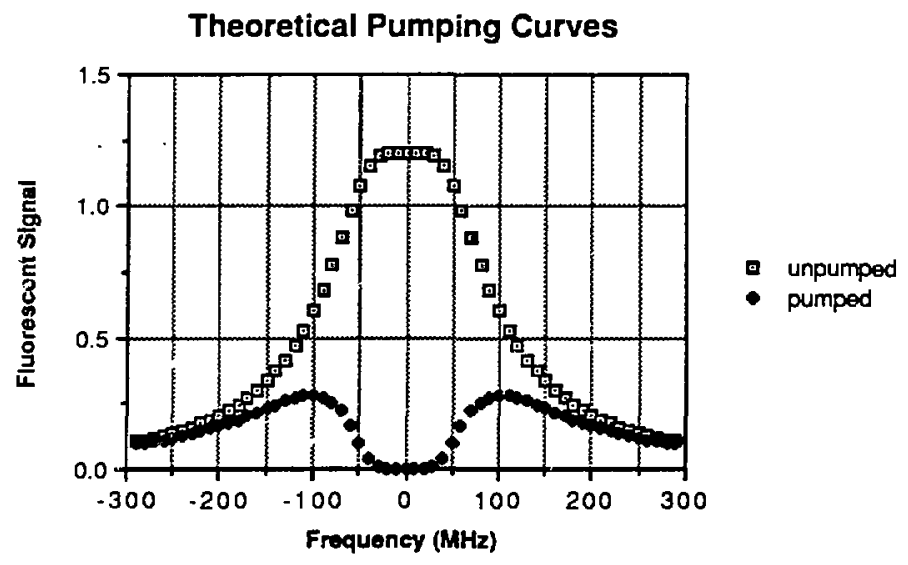

Figure III.11

\section{The Lasers}

The $\lambda=378 \mathrm{~nm}$ light 's produced in a krypton (Coherent 100-K3) pumped CW ringdye laser (Coherent CR699-21) using LD700 dye. The 6.1 watt rutput of the krypton laser $(647 \mathrm{~nm})$ is converted to approximately 1 watt of single mode $756 \mathrm{~nm}$ by the LD700 dye. The dye laser lases at $756 \mathrm{~nm}$ and an intracavity $\mathrm{LilO}_{3}$ (Coherent 7500-03) crystal doubles its frequency to $378 \mathrm{~nm}$ yielding an average of $5 \mathrm{~mW}$. This high $\mathrm{CW}$ second harmonic generation efficiency is a result of the high intracavity intensity.

We replaced the dye circulation system with non-standard components in order to minimize dye jet air bubbles which lead to power instabilities. As a further measure, we constantly monitor the dye laser's internal error signal and, in turn, disable data acquisition whenever the signal indicates a bubble or a mode instability. We found that a down time of approximately $100 \mathrm{msec}$ effectively in removes all traces of a laser instability from the data. 
The computer locks the frequency of the dye laser to the thallium $6 \mathrm{P}_{1 / 2}(\mathrm{~F}=1) \rightarrow$ $7 S_{1 / 2}(F=1)$ transition once every five cycles (every 5-10 minutes). It accomplishes this by scanning $1 \mathrm{CHz}$ up and down through the optical resonance and then calculating the left and right half-maximum points $v_{\mathrm{lhm}}$ and $v_{\mathrm{rhm}}$. Each scan is comprised of 320 points representing $1 \mathrm{msec}$ of data, taken $5 \mathrm{msec}$ apart. The final optical frequency is the average over both scans of the average of $v_{\mathrm{lhm}}$ and $v_{\mathrm{rhm}}$. Scanning the laser both up and down in frequency serves to reduce any hysteresis in the CR- 699 galvanometer that varies the optical path length in the laser cavity. To further reduce this problem, the computer always sets the final frequency moving in the same direction. After ten hours of warm-up, the laser typically drifts $10 \mathrm{MHz}$ between stabilizations which is acceptable in view of the transition's broadened linewidth of $\Delta \mathrm{v}_{\mathrm{eff}}=200 \mathrm{MHz}$.

The combined laser and optics configuration delivers a light beam with the following characteristics:

$$
\begin{aligned}
& \text { Wayelength } \lambda=378 \mathrm{~nm} \\
& \text { Power }=5 \text { milliwatts } \mathrm{CW} \pm 2.5 \% \mathrm{RMS} \text { at } 10 \mathrm{~Hz} \text { to } 100 \mathrm{kHz} \\
& \text { Nominal beam waist }=0.2 \mathrm{~cm} \\
& \text { Polarization } \varepsilon=\mathrm{z} \text { (95\% pure) }
\end{aligned}
$$

Of the $5 \mathrm{~mW}$ produced by the laser, about $4 \mathrm{~mW}$ reaches each interaction region, the rest being lost through reflections and scattering. With a beam waist of $w_{0}=.2 \mathrm{~cm}$, the intensity at the atomic beam is $\mathrm{I}_{378 \mathrm{~nm}} \approx 30 \mathrm{~mW} / \mathrm{cm}^{2}$. The laser beam is directed through the apparatus in an "open ring" configuration as shown in § III.A. 


\section{The Fluorescent Detectors}

Fluorescent Detector Schematic (not drawn to scale)

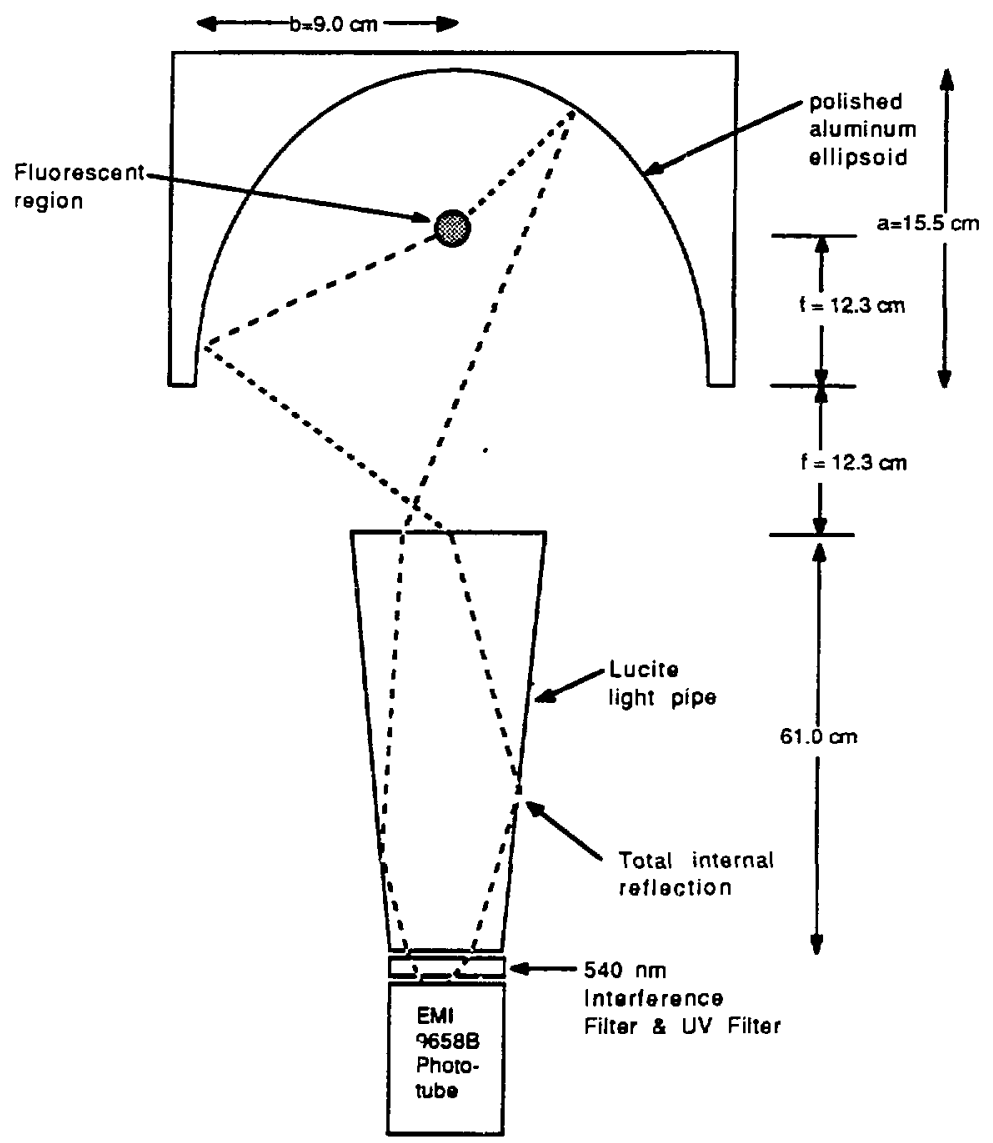

Figure III.12 
The $\lambda=535 \mathrm{~nm}$ fluorescent signal is measured by a detection system which we believe to be $4 \%$ efficient and composed of six parts:

\begin{tabular}{ll} 
component & $\frac{\text { efficiency }}{70 \%}$ \\
ellipsoidal reflector & $90 \%$ \\
lucite light-pipe & $80 \%$ \\
UV absorbing tïler & $40 \%$ \\
540 nm interference filter & $17 \%$ \\
EMI 9658B photorube & $4 \%$ \\
\hline overall efficiency & $4 \%$
\end{tabular}

We performed Monte Carlo computer simulations to optimize the efficiency of the system. A maximum overall efficiency and even efficiency across the atomic beam were achieved by varying the dimensions of the ellipsoid and of the light pipe.

The fluorescent light originates in at the intersection of the laser beam and the atomic beam in a cylindrical region $(0.1 \mathrm{~cm}$ thick $\times .2 \mathrm{~cm}$ radius $)$ at the focus of an aluminum ellipsoidal reflector. The ellipsoid (semi-major axis $\mathrm{a}=14.5 \mathrm{~cm}$ and semi-minor axis $\mathrm{b}=$ $9.0 \mathrm{~cm}$ ) should reflect about $70 \%$ of the light into the light-pipe.

A light-pipe conducts the fluorescence from the focus of the ellipsoid to the phototube, which is kept outside the magneric shields because of its slightly ferromagnetic components. The light-pipe is $61.0 \mathrm{~cm}$ long and slightly tapered to guide the light (dia. entrance $=7.6 \mathrm{~cm}$ and dia.exit $=4.4 \mathrm{~cm}$ ). Due to total internal reflection, light is only lost (about $10 \%$ ) at the faces of the pipe.

An uncoated UV filter $\left(10^{-4}\right.$ transmission at $378 \mathrm{~nm}$ ) blocks the unwanted $378 \mathrm{~nm}$ light accompanying the signal. It transmits about $80 \%$ of the $535 \mathrm{~nm}$ signal.

Because most light rays do not strike the interference filter at normal incidence, its center wavelength is slightly "red shifted" to $540 \mathrm{~nm}$. With a FWHM $=30 \mathrm{~nm}$, it is only $40 \%$ efficient. Its primary purpose is to block background light from the ovens and room 
lights.

An EMI 9658B bialkali phototube with a prismatic face converts the photons to electrons. With 11 dynodes engaged and 900 volts applied between the anode and cathode, it has a nominal gain of $10^{6}$. Its quantum efficiency at $535 \mathrm{~nm}$ is $17 \%$. 


\section{E. The Electric Field}

\section{The Electric Field Plates}

The extremely strong electric field, the central feature of any EDM experiment, is generated by a pair of carefully engineered plates that are oriented to produce a field in the z-direction. The two titanium electric field plates, each $100 \mathrm{~cm}$ long $\times 5.1 \mathrm{~cm}$ wide $\times 1.0$ $\mathrm{cm}$ thick, are held by titanium backing plates to form a $0.2 \mathrm{~cm}$ gap. Quartz spacers, ground to the nearest $.0005 \mathrm{~cm}$, both insulate and position the assembly. Brass "flowerpots", which are smooth, rounded discs of brass, are employed to position the quartz spacers in order to reduce the possibility of sparking from a sharp comer.

We use titanium for its high resilience to damage that can be caused by sparking between the plates at high voltages. Before using the plates at a particular voltage, they must first undergo a process called "conditioning" - temporarily increasing the voltage on the plates to induce sparking. This procedure should eliminate any surfaces irregularities that might cause leakage currents or sparking when the plates are uitimately used at the desired nominal voltage. In particular, before taking data at a field of $100 \mathrm{kV} / \mathrm{cm}$, we first set the field to $120 \mathrm{kV} / \mathrm{cm}$ for approximately 20 minutes. Once they are conditioned, the plates can hold up to $150 \mathrm{kV} / \mathrm{cm}$ under vacuum without obvious signs of sparking. As a precaution, it is advisable to take data at slightly lower fields.

Figure 111.13 is an end on view of the electric field plate assembly. An imaginary atomic beam is travelling into the page. The assembly includes the titanium plate, spacers, the backing plates, and the clamping plates. 
Electric Field Plate Assembly end viow

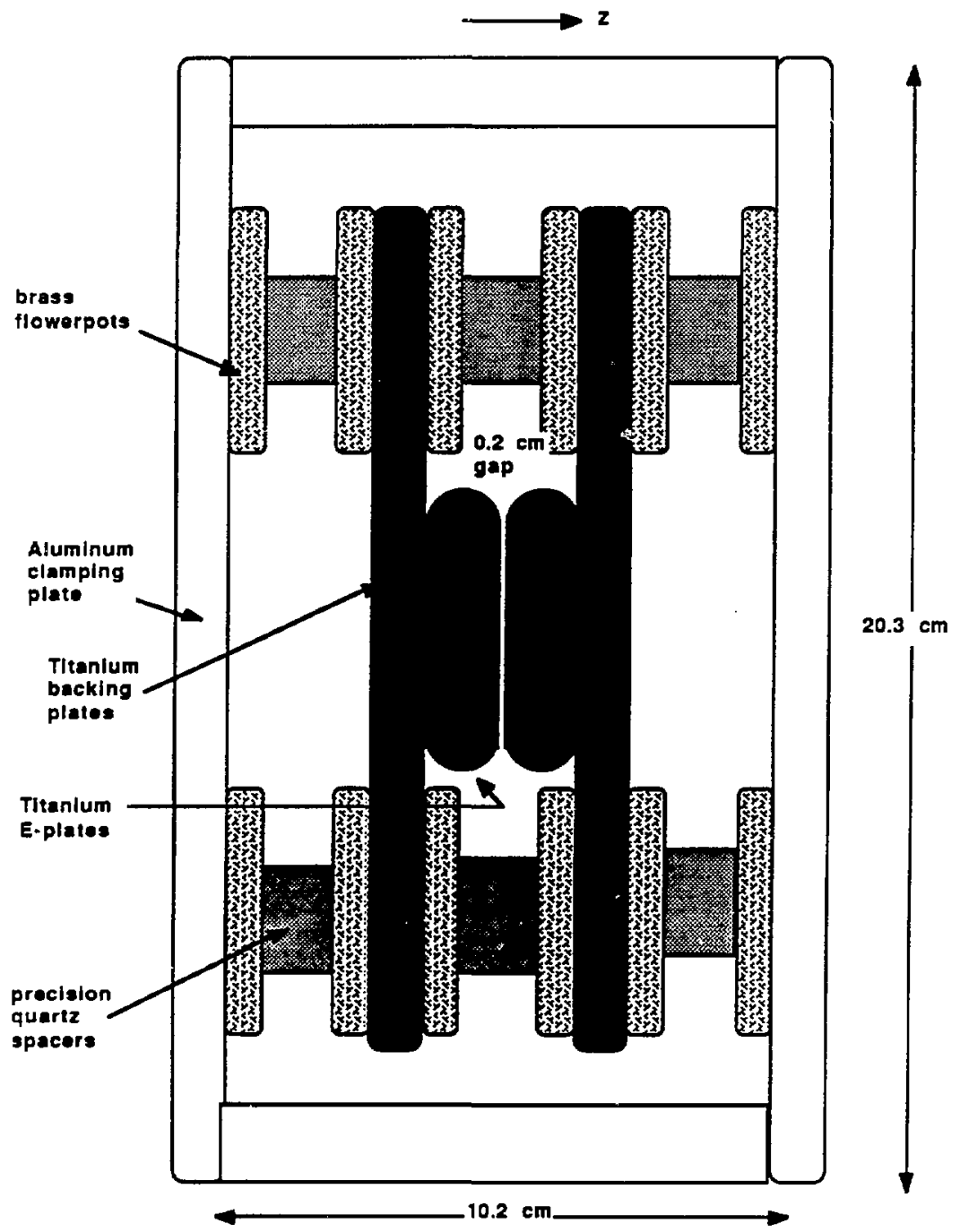

Figure III.13 


\section{The High Voltage Switch and Charging Time}

The high-voltage switch that connects the two 8-30 kV DC power supplies to the two electric field plates is constructed from over 48 individual reed relays. Because each relay is rated at $5 \mathrm{kV}$, eight relays are connected in series to act as a single SPST switch. Further, RC networks control the opening and closing of each relay so that no single relay "sees" more than $5 \mathrm{kV}$. Triggered by a TTL signal from the computer, the switch has a maximum switching rate of $10 \mathrm{~Hz}$.

This switch designed proved to be highly unreliable and was prone to failure and sparking. Plans are currently underway to develop an improved high-voltage switch.

The charging time of the plates is determined by a charging resistor $R=1 \mathrm{M} \Omega$ and the capaciance of $1500 \mathrm{~cm}$ of coaxial cable (approx. $1 \mathrm{pf} / \mathrm{cm}$ ) which yields $\tau=R C \approx 2 \mathrm{msec}$. We have neglected the capacitance of the plates which is

$$
\mathrm{C}_{\text {plates }}=\varepsilon_{0} \frac{\text { area }}{\text { spacing }}=230 \mathrm{pf}
$$

To ensure that no current flows which data is collected, each time the E-field reverses, the computer waits $100 \tau=200 \mathrm{msec}$ before taking data.

schematlc of reed-relay electric field switch

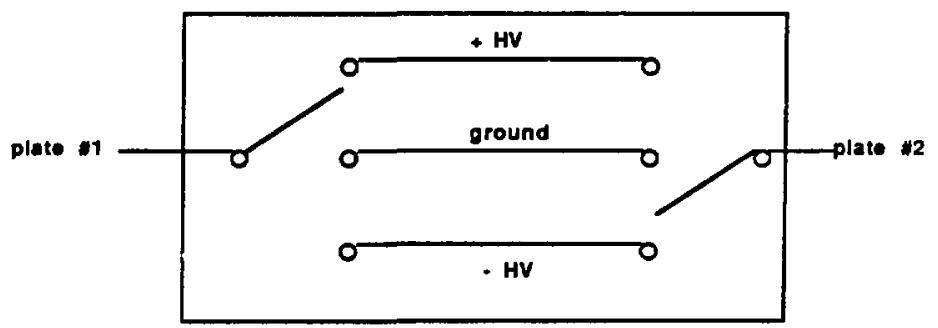

Figure III.14 


\section{F. The Magnetic Field}

\section{A Schematic of the Coils and Shields}

A constant, well defined magnetic field in the z-direction is an essential feature of the experiment in that it provides an axis of quantization for the atoms. Although the Ramsey technique is sensitiv only to the average magnetic field between the of loops, the direction of this field must be controlled so as to eliminate the vxE systematic, which arises from a misalignment between the electric and magnetic fields (see § III.J.1). The magnetic field coils are designed in such a way as to maximize the homogeneity of the magnetic field across the atomic beam. A more homogeneous B-field enables us to control systematics more easily. Finally, the main chamber and the coils are enclosed in three layer of mumetal shielding. Any fluctuations in the magnetic field that penetrate the shields will manifest themselves as noise in our EDM measurement through their interactions with an atom's magnetic dipole moment. Figure III. 15 is an end on, cross-section (looking down the $x$-axis) of the apparatus. 
The magnetic field coils and shielding

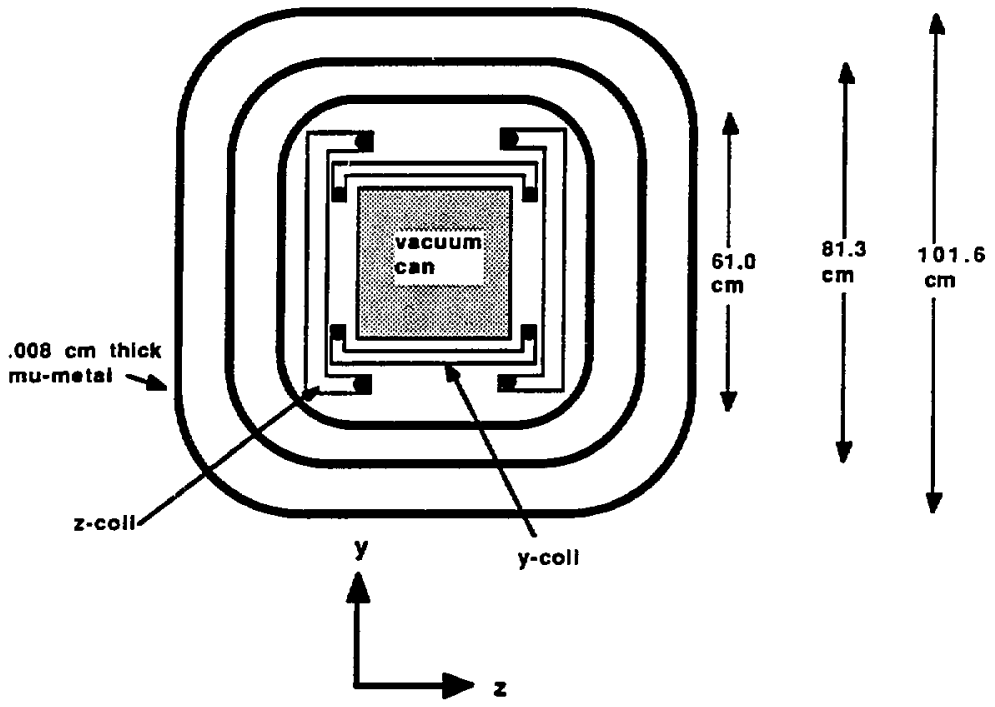

Figure III.15

\section{The Coils and Field Homogeneity}

The primary component of the static quantizing magnetic field $\overrightarrow{\mathrm{B}_{0}}$ is produced by a pair of coils oriented in the z-direction. The z-coils have 72 turns of wire and a total resistance $R=39.5$ ohms. A second pair of coils, the y-coils, compensates for any mechanical misalignment between the average electric field $\vec{E}$ and the average magnetic field $\overrightarrow{B_{0}}$ between the E-field plates. The y-coils are constructed of 18 turns of wire and have a combined resistance $R=10.0$ ohms.

The spacings and widths of the two coils are carefully designed to minimize 
inhomogeneities. More specifically, if $N$ is the width of a given rectangular coil, a spacing $d=\frac{w}{\sqrt{3}}$ between it and its partner will greatly reduce inhomogeneities in the B-field near the center of the pair. The optimal geometry is shown in figure III.16.

The Spacing of the "Helmholtz" coils

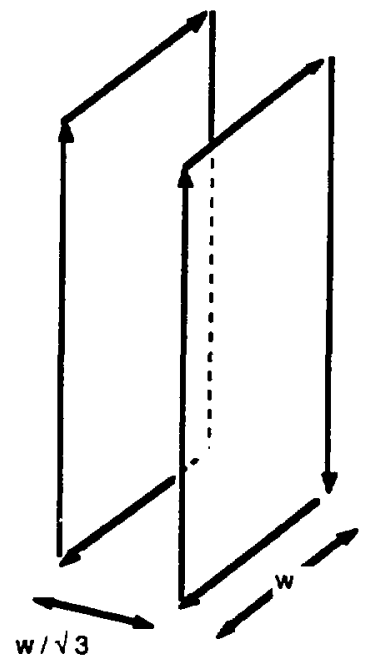

Figure III.16

It can be shown that this geomerry gives rise to inhomogeneities on the order of $\frac{\Delta \mathrm{B}}{\mathrm{B}}=\left(\frac{I}{\mathrm{~W}}\right)^{4}$ instead of $\left(\frac{\mathrm{I}}{\mathrm{W}}\right)^{2}$, where $\mathrm{r}$ is the radial distance from the center of the coils.

Both the $y$-coil and the z-coil are powered by North Hills constant current supplies which are characterized by drifts of $2 \mu$ amps /hour after days of warm-up. Empirically, the combined effect of the $z$-coil and the shields produces a B-field $\mid B_{0}$ (gauss) $I=2.8 \mathrm{I}_{z}$ (amperes).

A third pair of coils, the "trim" coils, have 13 turns each and cncompass the center of the region between the two ff loops. They are used to adjust the average B-field between 
the If loops. If the Ramsey interference fringes are to lie at the center of the Rabi pattern, it is essential that the average B-field matches the B-field inside each loop (see § II.H.1). The measured position of the Ramsey fringes, in the absence of the trim coil, indicates that $\left.\left|B_{\text {davg }}-\right| B_{0}\right|_{\text {rf }} \approx 2 \times 10^{-3}$ gauss - a small, but easily noticeable effect. It is found that applying the same current both to the $z$-coil and the trim coil $\left(I_{\text {trim }}=I_{z}\right)$ eliminates this difference.

\section{Mu-Metal Shielding}

To isolate the interaction region from both external time-varying and stationary magnetic fields, three layers of $.08 \mathrm{~cm}$ thick mu-metal (Hypernom) shielding enclose the main chamber and the three pairs of coils. Each shield has end-caps with openings through which the atomic beam passes. The openings are protected by mu-metal "necks" which are located inside the buffer chambers.

Mu-metal must be annealed before it is used as a shield. We purchased unannealed material, machined and rolled it, and finally thermally annealed it at Pyromet Industries in San Carlos, CA. The following heat annealing prescription was used. The material is first brought to $\mathrm{T}=2050^{\circ} \mathrm{F}$, where it remains for 4 hours. It is then rapidly cooled at a rate of $400^{\circ} \mathrm{F} /$ hour until $\mathrm{T}=800^{\circ} \mathrm{F}$ is attained, at which point it is permitted to cool freely at any rate. The entire process occurs in a hydrogen reducing atmosphere. Is is believed that the quick cooling stage "freezes" the magnetic domains into the randomized positions and, therefore, increases the final permeability.

We compared the permeabilities of several small, cylindrical pieces of annealed and unannealed mu-metal. By measuring the B-field at the center of the cylinder when it is immersed in a fairly uniform $B=1.75$ gauss, we deduced the shielding factor and, thus, the permeability of each sample. Figure III.17 shows our experimental set-up. 


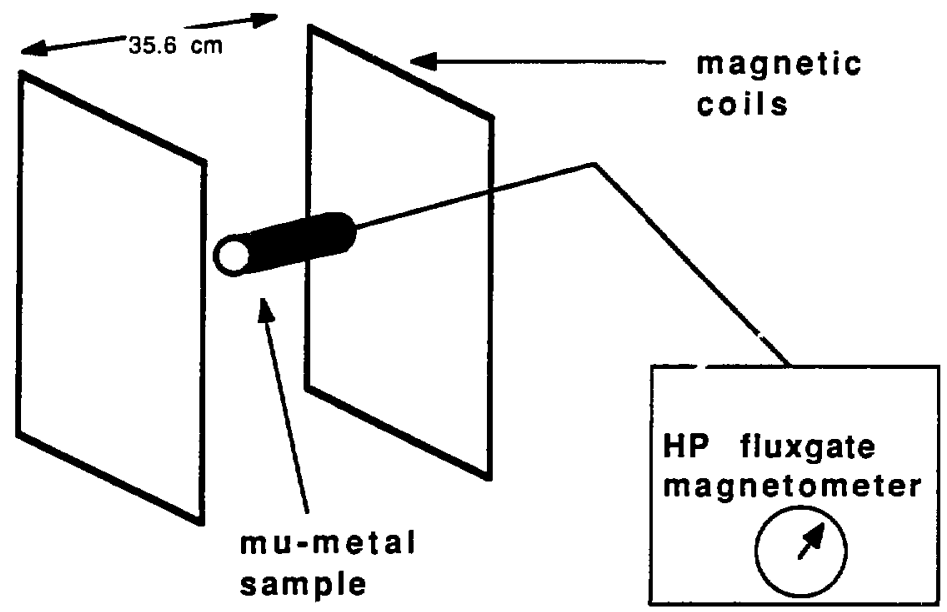

Figure III.17

If $B_{\text {ext }}=1.75$ gauss is the applied $B$-field and $B_{\text {in }}$ is the field at the center of the sample, then the permeability is

$$
\mu=\frac{B_{\text {ext }}}{B_{\text {in }}} \frac{L^{3 / 2}}{2 \sqrt{R}}
$$

where $L$ is the length of the cylinder, $t$ is its thickness, and $R$ is its radius. This formula is valid for $4<L / R<80$. We summarize our results in the following table:

\begin{tabular}{|c|c|c|c|c|c|c|}
\hline$L(\mathrm{~cm})$ & $\mathrm{R}(\mathrm{cm})$ & $\perp(\mathrm{cm})$ & $\mathrm{B}_{\mathrm{ext}}$ & $\mathrm{B}_{\text {in }}$ & ـ & \\
\hline 35.6 & 2.54 & .08 & 1.75 & .0045 & $3.4 \times 10^{5}$ & annealed \\
\hline 45.7 & 3.18 & .08 & 1.75 & .02 & $1.0 \times 10^{5}$ & annealed \\
\hline 55.9 & 3.81 & .08 & 1.75 & .023 & $1.1 \times 10^{5}$ & annealed \\
\hline 35.6 & 2.54 & .08 & 1.75 & .5 & $3.1 \times 10^{3}$ & unannealed \\
\hline
\end{tabular}

Figure III.18 
We conclude that annealling improves the permeability by a factor of roughly one-hundred and $\mu_{\text {annealed }}=2 \times 10^{5}$ and $\mu_{\text {unamnealed }}=3 \times 10^{3}$.

If the shielding is approximated as a two-dimensional problem, the effective shielding factor of three circular shield is

$$
A=\frac{u^{3} t^{3}}{8 d_{1} d_{2} d_{3}}\left(1-\frac{d_{1}^{2}}{d_{2}^{2}}\right)\left(1-\frac{d_{2}^{2}}{d_{3}^{2}}\right)
$$

where $\mu$ is the permeability, $t$ is the thickness, and $d$ is the diameter of each shield. Using the parameters $\mathrm{t}=.08 \mathrm{~cm}, \mathrm{~d}_{1}=61.0 \mathrm{~cm}, \mathrm{~d}_{2}=81.3 \mathrm{~cm}, \mathrm{~d}_{3}=101.6 \mathrm{~cm}$, and $\mu=2 \times 10^{5}$, we find an attenuation of $\mathrm{A}=2 \times 10^{5}$.

Once the mu-metal shields are annealed and situated, it is generally believed that they must be demagnetized to eliminate any residual magnetization that was "frozen" in during the annealling process. Demagnetization is accomplished by applying a large enough magnetic field to saturate the shields, and then slowly decreasing this field to zero while its direction is periodically reversed.

The magnisuc field is generated by eight water-cooled loops of $3 / 16^{\prime \prime}$ dia. copper tubing. The circuit formed by the loops travels down the outside of all three closed shields, up through the necks inside the vacuum chamber, and out again to close the circuit. The DC current in the loops is slowly reduced from 80 amps to 0 amps, while the direction of the current is reversed at a rate of $1 \mathrm{~Hz}$. I $=80$ amps corresponds to roughly $B=4000$ gauss inside the shielding. This may not fully saturate the mu-metal, but it probably suffices. 


\section{G. Radiofrequency Transitions}

\section{The "Hairpin" Loops}

The oscillating magnetic fields that cause transitions in the atoms are generated by two If loops that are situated at opposite ends of the main chamber. They are copper strips, $5 \mathrm{~cm}$ long, bent to form a loop through which the atomic beam passes and they produce a magnetic field in the $x$-direction of the form $\overrightarrow{B_{1}}=B_{1} \sin (\omega t+\alpha)$, where $B_{1}$ and $\alpha$ are the amplitude and phase, respectively of the if field. As will be further discussed in $\S$ III.H.2, the amount of if current applied to the loop is just enough to rotate the magnetic dipole moment of the atom 90 degrees. In practice, this current is 80 milliamperes.

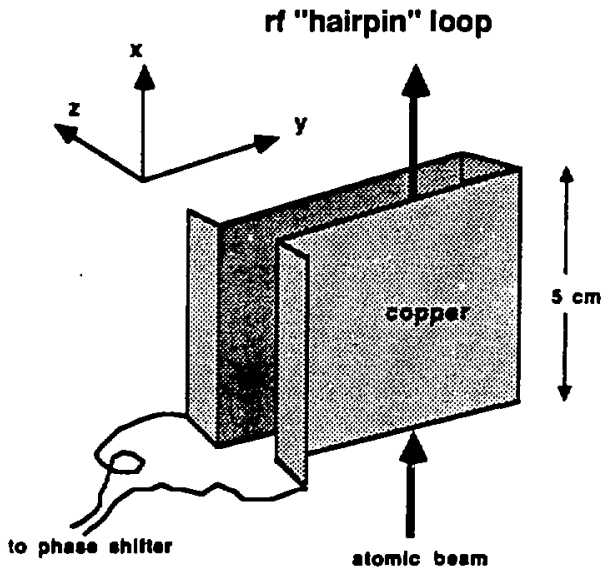

Figure III.19 


\section{The RF Synthesizer and Phase-Shifter Circuit}

A Hewlitt-Packard Model 8904A frequency synthesizer, with a frequency range of 0 $<v_{\mathrm{rf}}<600 \mathrm{kHz}$ and an amplitude range of $0-10$ volts into 50 ohms, generates the if waveform that is applied to the if loops. The computer controls the synthesizer's frequency via an IEEE-488 (GPIB) digital bus.

The output of the synthesizer is fed into a phase-amplitude shifter that was designed and built by the UC Berkeley Physics Dept. Electronics Shop. The shifter is responsible for amplifying and controlling the phase and amplitude of the if signal in each if loop. The relative phase between two loops is switchable among the six values $0, \pi / 2, \pm \pi / 4$, and $\pm 3 \pi / 4$ radians. Each loop's amplitude can be switched among off and two present levels. The setding ime for any cliange is less that $1 \mathrm{msec}$.

Although the shifter was designed to completely reverse the phase, non-negligible if loop inductances lead to phase errors on the order of \pm 30 milliradians. These small errors are removed by the chopping scheme presented in § III.I.2 (also see § III.J.3). Figure III. 19 is a schematic of the radiofrequency circuit.

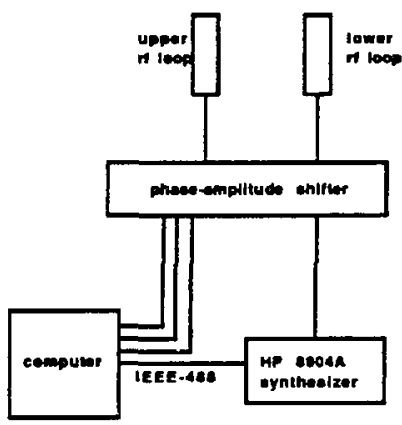

Figure III.19 


\section{Radiofrequency Transitions in the RF-Loops}

We now explore the behavior of an atom's statevector as it passes through a single if loop being driven with arbitrary power and phase. Our result will be a matrix, which we will in turn apply to the wavefunction to describe the magnetic dipole transition. This construction will be useful when we calculate the signal resulting from the complete twoloop magnetic resonance experiment.

In the laboratory frame, inside an rf loop, an atom sees a total magnetic field

$$
\vec{B}=B_{0} \hat{z}+B_{1} \cos (\omega t+\alpha) \hat{x}
$$

where $\mathrm{B}_{0}=.28$ gauss is the static average magnetic field applied in the $z$-direction. Here, $\alpha$ is the phase, $B_{1}$ is the magnitude, and $\omega$ is the frequency of the if field. The evolution of the $\mathrm{T}$ wavefunction $\psi$ for the $\mathrm{F}=1$ state is given by the Schrodinger equation:

$$
H \psi=-\mathrm{g}_{\mathrm{F}} \mu_{0} \overrightarrow{\mathrm{F}} \cdot \overrightarrow{\mathrm{B}} \psi=\mathrm{i \hbar} \frac{\partial \psi}{\partial \mathrm{t}} .
$$

which is equivalent to three coupled differential equations that can be solved analytically to give the familiar Rabi flopping formula.

However, an equivalent, geometric approach is just as accurate and more enlightening. If we consider a frame that is comoving with an atom and rotating about the $z$-axis with angular frequency $\omega$, the total effective magnetic field becomes

$$
\overrightarrow{B_{e f f}}=\left(B_{0}-\frac{\omega}{\gamma}\right) \hat{z}+B_{1} \cos \alpha \hat{x}-B_{1} \sin \alpha \hat{y}
$$

where $\gamma=g_{F} \mu_{0}$. This field is portrayed in Figure III.20 


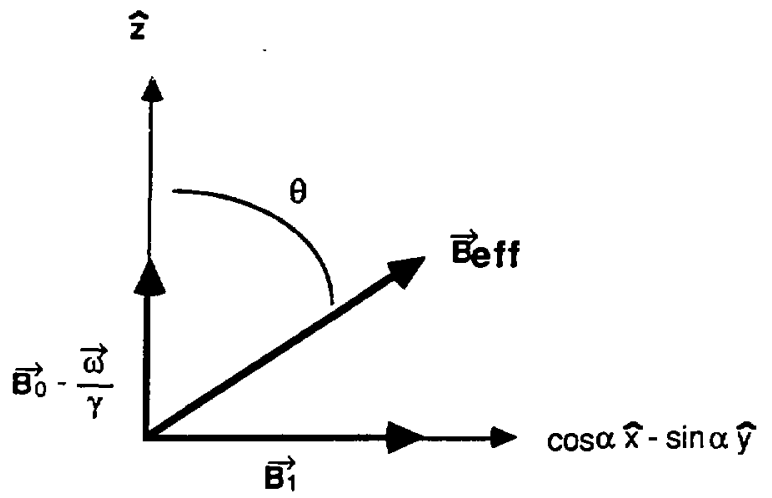

\section{The Magnetic Field in the Rotating Frame}

\section{Figure III.20}

The atom's magnetic dipole moment will precess about this field an angle

$$
\eta=\gamma B_{\text {eff }} \frac{L}{v}=\mu_{0} g_{F} B_{\text {eff }} \frac{L}{v}
$$

where $L=5 \mathrm{~cm}$ is the length of an $\mathrm{ff} l 00 \mathrm{p}$ and $v$ is the velocity of the atum. The angle between $\overrightarrow{B_{e f f}}$ and the $z$-axis is $\theta=\tan ^{-1}\left(\frac{B_{0}-\frac{\omega}{\gamma}}{B_{1}}\right)$ and the projection of $\overrightarrow{B_{e f f}}$ in the $x-y$ plane is an angle $\alpha$ away from the $y$-axis.

This is easy to understand classically - a magnetic dipole $\vec{\mu}$ precesses about a magnetic field $\vec{B}$ because it experiences a torque $\vec{\imath}=\vec{\mu} \times \vec{B}$. The total angle of precession is independent of $\vec{\mu}$ and is simply $\eta=|B| t$ where $t$ is period during which the torque acts on the dipole.

At this point we derive an expression for the $3 \times 3$ matrix operator $R(\alpha, \theta, \eta)$ which we will apply to a wavefunction $\psi$ to account for precession in the above defined effective magnetic field in the rotating frame. If we denote the atomic wavefunction before 
precession as $\psi_{\text {in }}$ and the outgoing wavefunction at $\psi_{\text {out }}$, then we have

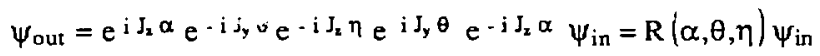

with $J_{x}, J_{y}$, and $J_{z}$ being any consistent representation of the $J=13 \times 3$ angular rotation matrices:

$$
\begin{aligned}
& \mathrm{e}^{\text {i J } \theta}=\left(\begin{array}{ccc}
\frac{1+\cos \theta}{2} & \frac{\sin \theta}{\sqrt{2}} & \frac{1-\cos \theta}{2} \\
-\frac{\sin \theta}{\sqrt{2}} & \cos \theta & \frac{\sin \theta}{\sqrt{2}} \\
\frac{1-\cos \theta}{2} & -\frac{\sin \theta}{\sqrt{2}} & \frac{1+\cos \theta}{2}
\end{array}\right) \\
& \mathrm{e}^{\mathrm{i} \mathrm{J}_{\mathbf{z}} \eta}=\left(\begin{array}{ccc}
\mathrm{e}^{\text {i }} & 0 & 0 \\
0 & 1 & 0 \\
0 & 0 & \mathrm{e}^{\cdot \text { i } \eta}
\end{array}\right)
\end{aligned}
$$

Using (III.G.6) and (III.3.7) for rotations about the $\mathrm{y}$ and $\mathrm{z}$ axes, we find

$$
\begin{aligned}
& e^{i J_{y} \theta} e^{-i J_{z} \alpha}=\text { henmetian conjugate }\left(e^{i} J_{1} \alpha e^{-i J_{y} \theta}\right) \\
& =\left(\begin{array}{ccc}
\frac{1+\cos \theta}{2} \mathrm{e}^{-i \alpha} & \frac{\sin \theta}{\sqrt{2}} & \frac{1-\cos \theta}{2} \mathrm{e}^{i \alpha} \\
-\frac{\sin \theta}{\sqrt{2}} \mathrm{e}^{-i \alpha} & \cos \theta & \frac{\sin \theta}{\sqrt{2}} \mathrm{e}^{i \alpha} \\
\frac{1-\cos \theta}{2} \mathrm{e}^{-i \alpha} & -\frac{\sin \theta}{\sqrt{2}} & \frac{1+\cos \theta}{2} \mathrm{e}^{i \alpha}
\end{array}\right)
\end{aligned}
$$

and a resulting matrix operator $R(\alpha, \theta, \eta)=$ 


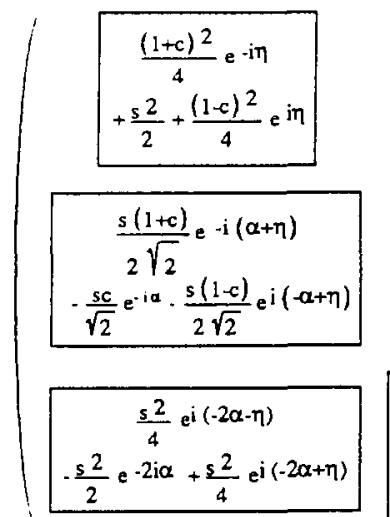

$$
\begin{gathered}
\frac{s(1+c)}{2 \sqrt{2}} e^{i(\alpha-\eta)} \\
-\frac{s c}{\sqrt{2}} e^{i \alpha-\frac{s(1-c)}{2 \sqrt{2}}} e^{i(\alpha+\eta)} \\
\hline
\end{gathered}
$$$$
\begin{gathered}
\frac{s^{2}}{4}=i(2 \alpha-\eta) \\
-\frac{s^{2}}{2} e^{2 i \alpha}+\frac{s^{2}}{4} e^{i(2 \alpha+\eta)} \\
\hline
\end{gathered}
$$$$
\begin{gathered}
\frac{s^{2}}{2} e^{-i \eta} \\
+c^{2}+\frac{s^{2}}{2} e^{i \eta} \\
\hline
\end{gathered}
$$$$
\begin{gathered}
\frac{s(1-c)}{2 \sqrt{2}} e^{i(\alpha-\eta)} \\
+\frac{s c}{\sqrt{2}} e^{i \alpha}-\frac{s(1+c)}{2 \sqrt{2}} e^{i(\alpha+\eta)}
\end{gathered}
$$

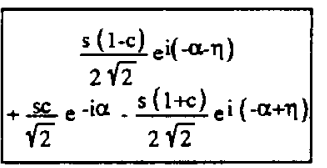

$$
\begin{gathered}
\frac{(1-c)^{2}}{4} e^{-i \eta} \\
+\frac{s^{2}}{2}+\frac{(1+c)^{2}}{4} e^{i n}
\end{gathered}
$$

where

$$
\begin{gathered}
s=\sin \theta=\frac{\gamma B_{1}}{\sqrt{(\Delta \omega)^{2}+\left(\gamma B_{1}\right)^{2}}} \\
c=\cos \theta=\frac{\Delta \omega}{\sqrt{(\Delta \omega)^{2}+\left(\gamma B_{1}\right)^{2}}} \\
\eta=\sqrt{(\Delta \omega)^{2}+\left(\gamma B_{1}\right)^{2}} \frac{L}{v}
\end{gathered}
$$

\section{The One-Loop Rabi Pattern}

The very first magnetic resonance experiments were performed using a single rf loop. It is instructive to consider such a situation in our current experimental configuration of polarizing and analyzing the atoms. In doing so, we will better appreciate the advantages of the Ramsey two-loop technique.

We showed in $\S$ III.C.I that our scheme of optical pumping drives an atom in an initial state

$$
\psi_{0}=\sqrt{\frac{7}{20}}\left(\begin{array}{l}
0 \\
1 \\
0
\end{array}\right)
$$

and gives a fluorescent signal proportional to $S=|a|^{2}+\mid d^{2}$ when we analyze an atom with 
wavefunction:

$$
\psi_{\mathrm{f}}=\left(\begin{array}{c}
\mathrm{a} \\
\mathrm{b} \\
\mathrm{c}
\end{array}\right)
$$

The final wavefunction of an atom undergoing the polarizer and one-loop if transition is

$$
\psi_{\mathrm{f}}=R(\alpha, \theta, \eta) \psi_{0}=R(\alpha, \theta, \eta) \sqrt{\frac{7}{20}}\left(\begin{array}{l}
0 \\
1 \\
0
\end{array}\right)
$$

and the corresponding analyzer signal is $S=1-\left.\left|b^{2}=1-\right| \Psi_{\mathrm{fq}}\right|^{2}$

$$
=\frac{7}{20}\left(1-\frac{s^{4}}{2} \cos 2 \eta-2 s^{2} c^{2} \cos \eta-\frac{s^{4}}{2}-c^{4}\right)
$$

If we choose an optimum if power such that $\sin \eta=1\left(\gamma B_{1}=\frac{\pi v}{2 L}\right)$ when $\omega=\omega_{0}$, and utilize the values of $L=5 \mathrm{~cm}$ and $\mathrm{v}=\overline{\mathrm{v}}=4.0 \times 10^{4} \mathrm{~cm} / \mathrm{sec}$, we calculate the following signal versus frequency (Figure III.21):

Calculation of One-Loop Pattern

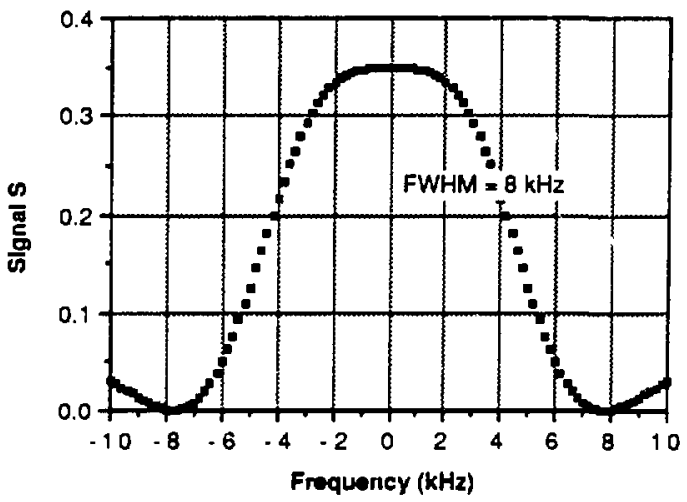

Figure IIL.21 
Experimental curves reproduce this shape and width exceedingly well with a measured FWHM $=8 \mathrm{kHz}$. The experimental one-loop pattern is shown below in Figure III. 22 .

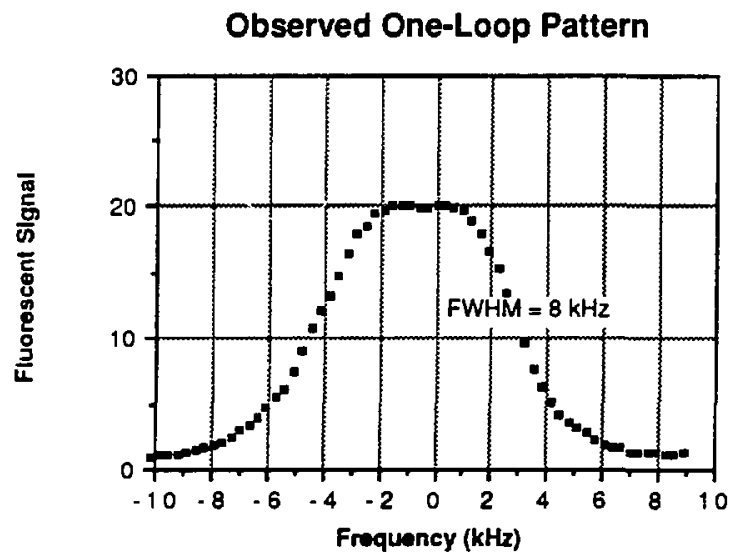

Figure II.22 


\section{H. Two-Loop Signals - the Evolution of an Atom's Wavefunction}

\section{The Signal for Arbitrary RF Phase, Power, and Frequency}

We now turn our attention to the actual experimental method we use in our search for the electron EDM - a two-loop Ramsey interference magnetic resonance measurement with the atom traversing a strong electric field between the two loops. In this section, we consider the very general case of any if phase difference between the if loops, any power, and any applied if frequency for a single velocity. Moreover, we make no assumptions about the applied radiofrequency field except that the power is the same in both loops.

Here the wavefunction at the analyzer is slightly more involved,

$$
\psi_{\mathrm{f}}=\mathrm{R}(\alpha, \theta, \eta) \zeta(\mathrm{E}, \Delta \omega) \mathrm{R}(0, \theta, \eta) \psi_{0}
$$

$\zeta(E, \Delta \omega)$ is an operator characterizing the effect on the wavefunction of the electric field and the average magnetic field between the loops. We can write it as

$$
\zeta(E, \Delta \omega)=\left(\begin{array}{ccc}
\mathrm{e}^{-i U} & 0 & 0 \\
0 & \mathrm{e}^{\mathrm{i} \varphi} & 0 \\
0 & 0 & \mathrm{e}^{\mathrm{i} u}
\end{array}\right)
$$

This operator is diagonal because the electric and magnetic fields cause no transitions between levels, rather they contribute phase-shifts that are detected through interference. The term $u$ includes the electron EDM phase-shift, any vxE effect, and any off-resonant magnetic field-dipole interaction:

$$
u=\varepsilon_{E D M}+\varepsilon_{v \times E}+\Delta \omega \frac{L_{r f}}{v}
$$


$\mathrm{L}_{\mathrm{rf}}=130 \mathrm{~cm}$ is the distance between the rf loops. The above $\varphi$ term represents the hyperfine structure induced quadratic Stark shift, a shift in energy of the $m_{F}=0$ state relative to the $m_{\mathrm{F}}= \pm 1$ states that is quadratic in the electric field. Its value has been measured in the past and it is found to be $\varphi=\frac{2 \pi \delta\left(E^{2}\right) L_{E}}{v}$ with the energy shift $\delta\left(E^{2}\right)=490 \mathrm{~Hz}$ when $E=100 \mathrm{kV} / \mathrm{cm}$.

We now calculate the population of the $\mathrm{m}_{\mathrm{F}}=0$ component of $\psi_{\mathrm{f}}$ :

$$
\begin{aligned}
& \mid \psi_{f, q^{2}}=\left\{\begin{array}{c}
s^{2} \cos (u+\alpha)\left[c^{2}(1-\cos \eta)^{2}-\sin ^{2} \eta\right] \\
+2 s^{2} \sin (u+\alpha)[\operatorname{csin} \eta(1-\cos \eta)] \\
+\cos \varphi\left(c^{2}+s^{2} \cos \eta\right)^{2}
\end{array}\right\}^{2} \\
& +\sin ^{2} \varphi\left(c^{2}+s^{2} \cos \eta\right)^{4}
\end{aligned}
$$

which we convert into a fluorescent signal $S=1-\psi_{f, d^{2}}$ and cast into the convenient form (after a great deal of algebra):

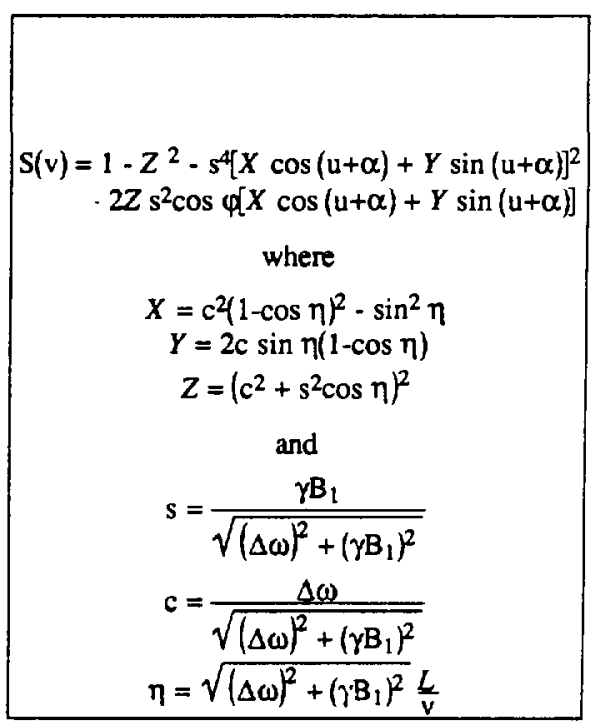


To recapitulate, we have derived an expression for the signal for any given if power, any if phase, and any frequency for an atom travelling with velocity v. If $S(v)$ is plotted against frequency $\Delta \omega$, the resulting graph is a series of interference fringes modulated by an overall one-loop envelope. This characteristic plot is call a "Ramsey pattem". When a monoenergetic atomic beam is used, the fringes are distinct and fill the whole Rabi envelope. On the other hand, only the first few central fringes are apparent when a beam with a full velocity distribution is used. Whereas the full-width of the modulating envelope is roughly $8 \mathrm{kHz}$, each fringe has a full-width on the order of only $70 \mathrm{~Hz}$.

For an atomic beam with a full velocity distribution, the position of the interference fringes on the overall Rabi envelope is a measure of how well $\left|\mathrm{B}_{\mathrm{d}}\right|_{\text {avg }}$ coincides with $\left|B_{d}\right|_{\text {upper } f f}$ and $\left|B_{d}\right|_{\text {lower } r}$. Only if the three are equal, will the fringes lie at the center of the Rabi pattem. Similarly, the relative positions (in frequency) of each one-loop Rabi

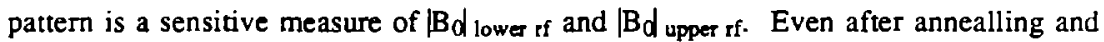
demagnetizing the mu-metal shields, we find the one-loop patterns separated by $400 \mathrm{~Hz}$.

\section{The Signal Near Resonance - the Central Fringe}

\section{Approximation}

The above expression for the signal is more general than we need because we always operate on the central fringe, or "white-light" fringe. At this point, we make an excellent, simplifying appmximation and also begin discussing velocity-averaged quantities to make comparisons with the experiment.

If we restrict ourselves to $100 \mathrm{~Hz}$ on each side of the resonance, it is not unreasonable to set $s=1$ and $c=0$ in the above calculations:

$$
s=\sin \theta=\frac{\gamma B_{1}}{\sqrt{(\Delta \omega)^{2}+\left(\gamma B_{1}\right)^{2}}} \approx 1
$$


because $(\Delta \omega)^{2} \ll\left(\gamma \mathrm{B}_{1}\right)^{2}$ and similarly for $\mathrm{c}=\cos \theta$. The signal for a given velocity is then

$$
S(v)=1-\cos ^{4} \eta-\left[\sin ^{2} \eta \cos (u+\alpha)\right]^{2}+2 \sin ^{2} \eta \cos ^{2} \eta \cos \varphi \cos (u+\alpha)(\text { III.H. } 7)
$$

which we rewrite in the final form:

$$
\begin{gathered}
S(v)=A+B \cos ^{2}(u-\alpha)+C\left(E^{2}\right) \cos (u-\alpha) \\
\text { where } \\
A=1-\cos ^{4} \eta \\
B=-\sin ^{4} \eta \\
C\left(E^{2}\right)=2 \sin ^{2} \eta \cos ^{2} \eta \cos \varphi\left(E^{2}\right) \\
\eta=\sqrt{(\Delta \omega)^{2}+\left(\gamma B_{1}\right)^{2} L} \frac{v}{v} \\
u=\varepsilon_{\mathrm{EDM}}+\varepsilon_{\mathrm{vXE}}+\Delta \omega \frac{L_{-\tau}}{v}
\end{gathered}
$$

This expression is exceptionally useful in that it separates the background and the quadratic Stark shift from the EDM term. It can also be integrated over the atomic beam's velocity distribution to give an accurate Ramsey pattern for any relative phase and if power. This formula agrees well with the measured patterns - figure III.23 shows one such pattern for $\pm 3 \pi / 4$ relative phase and zero electric field: 


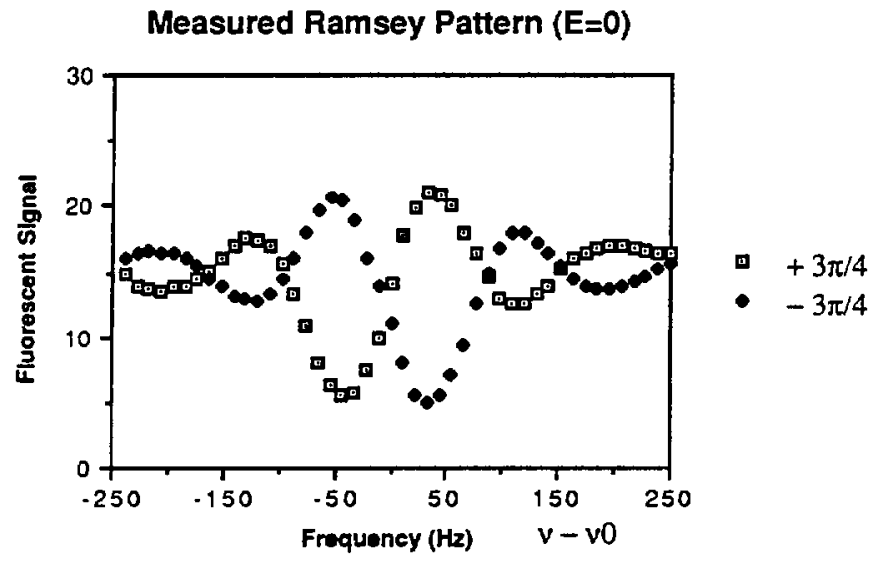

Figure III.23

The last expression we derived for the signal is appropriate when $\Delta \omega \approx 2 \pi \times 200 \mathrm{~Hz}$; it provides a simple picture of the signal for each of phase when the if frequency is properly set. When $\Delta \omega \approx 0$, it is safe to assume $\mathrm{u} \approx 0$ because the EDM and vxE phaseshifts are normally very small. With this, we can approximate the trigonometric functions:

$$
\begin{gathered}
\cos ^{2}(u-\alpha)=\cos ^{2} \alpha+2 u \cos \alpha \sin \alpha \\
\cos (u-\alpha) \approx \cos \alpha+u \sin \alpha
\end{gathered}
$$

to give a signal on resonance of

$$
S(v, u \sim 0)=A+B\left[\cos ^{2} \alpha+2 u \cos \alpha \sin \alpha\right]+C\left(E^{2}\right)[\cos \alpha+u \sin \alpha]
$$




\section{Setting the RF Power and Frequency}

From a theoretical point of view, the parameter $\eta(v)$, which depends on the applied if power $B_{1}$ and the atomic velocity, is optimal when our signal is most sensitive to an electron EDM and least sensitive to unwanted effects such as the quadratic Stark shift. In addition, we prefer an if power that minimizes the background term. The best situation would be to set $\eta(v)=\pi / 2$ for each velocity as it would maximize $B(v)$ and minimize $A(v)$ and $\mathrm{C}(v)$; however, this is impossible because our atomic beam contains the full velocity distribution at all times.

We performed computer calculations to estimate the velocity averaged parameters $\langle A\rangle,\langle B\rangle$, and $\langle C>$ in the absence of an electric field and as a function of $\mathrm{rf}$ power. By setting $\eta(v=\bar{v})=\pi / 2$, where $\bar{v}$ is the average velocity in the beam, we find

$$
\begin{aligned}
& \langle A\rangle=.88 \\
& \langle B\rangle=-.65 \\
& \langle C\rangle=.22
\end{aligned}
$$

In practice, we first measure a one-loop Rabi "bump" and vary the if power until the "bump" is pronounced, but not excessively flat on the top. We then reconnect the second loop and vary the power slightly until maximum Ramsey fringe contrast is attained for 0 phase-shift.

Every few minutes the computer measures the resonant rf frequency $\omega_{0}$ and corrects the applied frequency $\omega$. This is accomplished by establishing a $40 \mathrm{~Hz}$ window centered on $\omega_{0}$ and having the computer scan the full $40 \mathrm{~Hz}$ with $\alpha=+3 \pi / 4$ and $-3 \pi / 4$ taking 80 points each. As can be verified with $S(v)$, the two scans intersect at $\omega=\omega_{0}$. The computer fits each scan to a line and then calculates the intersection of the two fitted lines, thereby making maximum use of the data. The typical drift of the center $f$ frequency is about $2 \mathrm{~Hz}$ hour after days of warm-up for the constant current power supplies. 


\section{Extracting the EDM for the Raw Signal}

\section{The Form and Size of the EDM, the vxE, and Magnetic Noise Phase-Shifts}

Before examining the manner in which we extract the electron EDM from the signal, we first present expressions for the phase-shifts resulting from three major contributions w the signal $S(v)$ through the $u$ term in (III.H.8) - the EDM, the vxE effect, and $\Delta \omega$ magietic noise. Although, it may seem a bit premature to discuss the vxE systematic, understanding its behavior under the various chops is essential to comprehending the reason for our data collection technique. The vxE systematic is fully covered in Section III.J.1 and the problem of magnatic noise is addressed in § III.K.3. Having established formulae for each of the three phase-shifts, we will then make some order of magnitude comparisons. We will use $\mathrm{v}=\overline{\mathrm{v}}=4 \times 10^{4} \mathrm{~cm} / \mathrm{sec}$ for the velocity and $\mathrm{E}=100 \mathrm{kV} / \mathrm{cm}$ for the electric field throughout this discussion.

The interaction energy of an electric dipole with an electric field is simply $H=-\vec{d} \cdot \vec{E}$ and, accordingly, the Tl atomic EDM interacts with an energy

$$
H=-\overrightarrow{\mathrm{d}_{\text {atom }}} \cdot \overrightarrow{\mathrm{E}}=-\mathrm{R} \overrightarrow{\mathrm{d}_{\mathrm{e}}} \cdot \overrightarrow{\mathrm{E}}
$$

with $R$ being the enhancement factor (see \& I.C.2). We drop the vector signs and convert this into a phase-shift by multiplying by the time spent in the electric field and dividing by Planck's constant:

$$
E_{E D M}=-\frac{d_{e} R E / R / L_{E}}{\hbar v}
$$

The charge of the electron is used to convert the units of $d_{e}$ from c.g.s to e-cm and we attempt to keep track of the sign of the EDM. This is a central, often-used expression. Its numerical value is 


$$
\varepsilon_{\text {EDM }}(\text { radians })=-\frac{(-560)(330}{\left(10^{-27} \mathrm{erg}-\mathrm{sec}\right)\left(4 . \times 10^{4} \mathrm{~cm} / \mathrm{sec}\right)} \mathrm{stvolt/ \textrm {cm } ) ( 4 . 8 \times 1 0 ^ { - 1 0 } \mathrm { esu } ) ( 1 0 0 \mathrm { cm } )} \mathrm{d}_{\mathrm{e}}(\mathrm{e}-\mathrm{cm})
$$

or

$$
E_{\text {EDM }}(\text { radians })=2.2 \times 10^{20} \mathrm{~d}_{\mathrm{e}}(\mathrm{e}-\mathrm{cm})
$$

We see that an electron EDM of $d_{e}=10^{-28} \mathrm{e}-\mathrm{cm}$ corresponds to a phase shift of $2.2 \times$ $10^{-8}$ radians or an energy shift of $9 \times 10^{-33}$ ergs - a very small effect indeed. It is interesting to note that, while the interaction energy of an EDM is independent of velocity, the phase-shift scales as $1 / v$.

However, the phase-shift of the vxE effect is independent of velocity; this is instrumental in the separation of the two effects. It is

$$
\begin{gathered}
\varepsilon_{\mathrm{vxE}}(\text { radians })=\frac{2 \pi \mu_{0 \mathrm{gF}} E L_{E}}{c} \Theta=\frac{2 \pi\left(1.4 \times 10^{6} \mathrm{~Hz} / \text { gauss }\right)(330 \mathrm{statvol} / \mathrm{cm})(100 \mathrm{~cm})}{3\left(3 \times 10^{10} \mathrm{~cm} / \mathrm{sec}\right)} \Theta \\
=3.2 \theta \text { (radians) }
\end{gathered}
$$

where $\Theta$ is the is the angle between the average magnetic field $\overrightarrow{B_{0}}$ and the electric field $\vec{E}$.

When the applied if frequency $\omega$ is not exactly equal to the resonant frequency $\omega_{0}=\mu_{0} g_{F} B_{0}$ through magnetic noise or an inaccurate setting of the if synthesizer, there will be an additional "off-resonant" phase-shift, proportional to $1 / v$, of the form:

$$
\varepsilon_{\text {mag }}=\Delta \omega \frac{L_{\mathrm{rf}}}{v}=\left(\omega-\omega_{0}\right) \frac{L_{\mathrm{rf}}}{\mathrm{v}}=\left(\omega-\mu_{0} g_{F} B_{0}\right) \frac{L_{\mathrm{Tf}}}{\mathrm{v}}
$$

which has numerical values 


$$
\varepsilon_{\text {mag }} \text { (radians) }=\frac{2 \pi(130 \mathrm{~cm})}{4 \times 10^{4} \mathrm{~cm} / \mathrm{sec}} \Delta v=2 \times 10^{4} \Delta v(\mathrm{~Hz})
$$

or, for a magnetic field fluctuation,

$$
\varepsilon_{\text {mag }}(\text { radians })=\frac{2 \pi\left(1.4 \times 10^{6} \mathrm{~Hz} / \text { gauss }\right)(130 \mathrm{~cm})}{3\left(4 \times 10^{4} \mathrm{~cm} / \mathrm{sec}\right)} \Delta \mathrm{B}=1 \times 10^{4} \Delta \mathrm{B} \text { (gauss) }
$$

At first glance, given the above estimates, the experiment appears impossible. In order to reach a precision of $\sigma_{\mathrm{C}}=1 \times 10^{-28} \mathrm{e}-\mathrm{cm}$, the electric and magnetic fields must be aligned to $\Theta<7 \times 10^{-9}$ radians and magnetic field fluctuations must be reduced to $\Delta \mathrm{B}<2.2 \times 10^{-12}$ gauss. Even a precision of $\sigma_{\mathrm{d}}=1 \times 10^{-24} \mathrm{e}-\mathrm{cm}$ is unlikely.

However, chopping various experimental pari meters enables us to separate the three effects with a great deal of precision.

\section{The RF Phase Chop, the E-Field Chop, and the Beam Chop}

To extract $d_{e}$ from the raw signal and to reduce systematics and noise, we use a lockin technique of chopping and building asymmetries. In all, we have sixteen different configurations arising from four if phases, two electric field directions, and two atomic beam directions. We presently concern ourselves with the purpose of the chops and postpone discussion of their exact ordering to $\S$ IV.A.

The sign of each of the three major contributions to the atomic wavefunction phaseshift $u$ depends critically on the directions of the E-field and atomic beam because they are functions of $\vec{E}$ and $\frac{1}{\vec{v}}$. 


$\begin{array}{ccccc}\vec{E} & \text { beam } & \varepsilon_{\mathrm{EDM}} & \varepsilon_{\mathrm{vxE}} & \varepsilon_{\mathrm{mag}} \\ ++ & \Uparrow & + & + & + \\ - & \Uparrow & - & - & + \\ + & \Downarrow & + & - & + \\ - & \Downarrow & - & + & +\end{array}$

\section{Figure III.21}

Figure III. 21 indicates that the electric field reversal is useful in extracting the EDM from magnetic field fluctuations. In fact, it is the only means we have of removing magnetic noise. It is therefore beneficial to chop $\mathrm{E}$ as fast as possible and to avoid resonances such as $60 \mathrm{~Hz}$, but technical considerations force us to chop $E$ with a period of $E_{\text {period }}=1.6$ seconds. The $E$ chop also removes the effects of any phase errors in the relative phase between of loops. The vxE systematic is removed with the beam chop which has a period of $B_{\text {period }}=25.6$ seconds.

The If phase is rapidly chopped among the four values $\pm \pi / 4 \pm 3 \pi / 4$ at a rate of $\mathrm{Rf}_{\text {period }}=400 \mathrm{msec}$ ( $100 \mathrm{msec} /$ phase). This chop is extremely useful in reducing all non-magnetic noise sources. In addition, it eliminates any real possibility of a false effect arising though an incomplete E reversal combined with the quadratic Stark effect. To explore this, wa focus our attention on upgoing beam and utilize our expression for the signal on resonance (III.H.11):

$$
S_{\Uparrow}(u=0)=A_{\Uparrow}+B_{\Uparrow}\left[\cos ^{2} \alpha+2 u \cos \alpha \sin \alpha\right]+C_{\Uparrow}\left(E^{2}\right)[\cos \alpha+u \sin \alpha] . \text { (III.I.9) }
$$

For the if phases in question, 


$$
\begin{aligned}
& S_{\Uparrow, \pm \frac{\pi}{4}}(u=0)=A_{\Uparrow}+B_{\Uparrow}\left(\frac{1}{2} \pm u\right)+\frac{C_{\Uparrow}\left(E^{2}\right)}{\sqrt{2}}(1 \pm u) \\
& S_{\Uparrow, \pm \frac{3 \pi}{4}}(u=0)=A_{\Uparrow}+B_{\Uparrow}\left(\frac{1}{2} \mp u\right)+\frac{C_{\Uparrow}\left(E^{2}\right)}{\sqrt{2}}(-1 \pm u)
\end{aligned}
$$

which, when judiciously added and subtracted, form a signal $\mathbf{S}$ that is independent of the quadratic Stark term $\mathrm{C}\left(\mathrm{E}^{2}\right)$ :

$$
\left.S_{\Uparrow}(+E)=S_{\Uparrow,+\frac{\pi}{4}}(+E)+S_{\pi_{0}-\frac{3 \pi}{4}(+E)-S_{\Uparrow,-} \frac{\pi}{4}(+E)-S_{\Uparrow,+}} \frac{3 \pi(}{4}+E\right)=\left(4 A_{\Uparrow}+2 B_{\Uparrow}\right)+4 B_{\Uparrow} u .
$$

We then build an asymmetry $\Delta$ in the electric field,

$$
\Delta \Uparrow=\frac{S_{\Uparrow}(+E)-S_{\Uparrow}\left(-E_{i}\right)}{S_{\Uparrow}(+E)+S_{\Uparrow}(-E)}=\frac{2 B_{\Uparrow}}{2 A_{\Uparrow}+B_{\Uparrow}}\left(\varepsilon_{E D M}+\varepsilon_{V X E}\right)=\Pi_{\Uparrow}\left(\varepsilon_{E D M}+\varepsilon_{V X E}\right)
$$

and a similar expression for the downgoing beam, with the critical difference in the sign of the vxE term:

$$
\Delta \Downarrow=\frac{S_{\Downarrow}(+E)-S_{\Downarrow}(-E)}{S_{\Downarrow}(+E)+S_{\Downarrow}(-E)}=\frac{2 B_{\Downarrow}}{2 A_{\Downarrow}+B_{\Downarrow}}\left(\varepsilon_{E D M}-\varepsilon_{V X E}\right)=\Pi_{\Downarrow}\left(\varepsilon_{E D M}-\varepsilon_{V X E}\right)
$$

The new factors $\Pi$ that multiply the EDM and $v x E$ phase shifts are called "analyzing powers". They are not necessarily equal for the two opposing beams because factors such as differences in velocity distribution and detector background will lead to a situation where $A_{\Uparrow} \neq A_{\Downarrow}$ and $B_{\Uparrow} \neq B_{\downarrow}$ Once the analyzing powers are established, separating the EDM from the vxE becomes the simple sum-difference equations:

$$
\varepsilon_{\mathrm{EDM}}=\frac{1}{2}\left(\frac{\Delta_{\Pi}}{\Pi_{\Pi}}+\frac{\Delta \Downarrow}{\Pi_{\Downarrow}}\right) \text { and } \varepsilon_{\mathrm{v} \times \mathrm{E}}=\frac{1}{2}\left(\frac{\Delta \pi}{\Pi_{\Pi}}-\frac{\Delta \Downarrow}{\Pi_{\Downarrow}}\right)
$$


or, employing the formulae for the phase shifts,

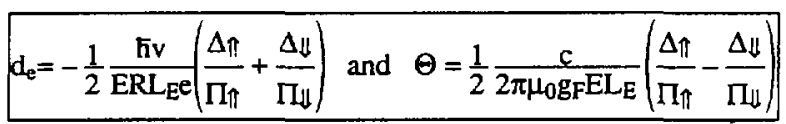

\section{Analyzing Powers and their Calibration}

At this point, it might appear as though we require precise calculations of $\mathrm{A}$ and $\mathrm{B}$ for each atomic beam in order to deduce the electric dipole moment from the signal. This problem is bypassed by actually measuring the "analyzing powers" $\Pi_{\Uparrow}$ and $\Pi \Downarrow$ for the upgoing and downgoing atomic beams.

They are measured, or calibrated, in a procedure in which the electric field reversal is replaced by a known of frequency shift. Keeping all other chops and data treatment the same, the analyzing powers are simply

$$
\begin{gathered}
\Pi_{\Uparrow}=\frac{\Delta \Uparrow}{u_{c a l}} \text { and } \Pi_{\Downarrow}=\frac{\Delta \Downarrow}{u_{c a l}} \\
\text { where } \\
u_{c a l}=2 \pi \Delta v_{c a l} \frac{L_{r f}}{v}
\end{gathered}
$$

We use a calibration if frequency shift $\Delta v_{\text {cal }}=1 \mathrm{~Hz}$ or $\mathrm{u}_{\mathrm{cal}}=.02$ radians. The degree to which the analyzing powers are measured is the degree to which the vxE effect is canceled. Typically we measure the analyzing powers to $2.5 \%$ which reduces the vxE effect by a factor of forty.

It is desirable not only to know the analyzing powers well, but also to maximize them. Higher analyzing powers lead to a more sensitive experiment. In $\S$ III.H.3, we showed that numerical simulations give the approximate values, 


$$
\langle A\rangle=.9 \text { and }\langle B\rangle=-.7
$$

which when substituted into (III.I.14), give an analyzing power $\Pi \approx-1.2$.

Background levels in our experiment reduce this to a measure value $\Pi_{\text {observed }} \approx-.75$. We have an additional fluorescent background of about $25 \%$ of the original rf resonance signal (see $\S$ III.K.5). This $25 \%$ must be added to A, which accounts for any signal that is independent of $u$. - 'is modification gives a more realistic analyzing power $\Pi \approx-.9$.

Regardless of its size, an accurate measurement of the analyzing powers allows us to honestly assess the sensitivity of the experiment and to separate the EDM from the $v x E$ effect. 


\section{J. Systematics}

\section{The $v \times E$ effect}

The vxE effect, the primary and, perhaps, the most predictable systematic we encounter in a search for the electron EDM, is due to the $\vec{\mu} \cdot B_{\text {total }} \overrightarrow{\text { interaction of an }}$ atom's magnetic dipole moment with the relativistic magnetic field seen by a moving atom in a strong electric field. We counter this systematic in two ways: we adjust the magnetic field to reduce it, and remove it from the data by chopping the direction of the atomic beam. In addition, our atomic beams travel in the vertical direction - this eliminates the problem of different trajectories for the different velocities that is caused by gravity.

If the z-axis is defined by the direction the the average electric field $\vec{E}=E_{0} \hat{z}$, then the total magnetic field in a frame that is comoving with the atom is

$$
\overrightarrow{B_{\text {total }}}=\overrightarrow{B_{0}}+\frac{1}{c} \vec{v} \times \vec{E}
$$

or

$$
\overrightarrow{B_{\text {totsl }}}=\left(B_{0 x}+\frac{v_{y} E_{0}}{c}\right) \hat{x}+\left(B_{0 y}-\frac{v_{x} E_{0}}{c}\right) \hat{y}+B_{0 z} \hat{z}
$$

where $B_{0}$ is applied magnetic field averaged over the length of the electric field plates. The magnitude of the total B-field about which the atom precess is

$$
\left|B_{\text {total }}\right|=\sqrt{B_{0 x}^{2}+B_{0 y}^{2}+B_{0 z}^{2}+\frac{2 B_{0 x} v_{y} E_{0}}{c}-\frac{2 B_{0 y} v_{x} E_{0}}{c}}
$$

If we neglect $v_{y}$ in favor of $v_{x}$, and expand the square rout, we find 


$$
\left|B_{\text {total }}\right|=B_{0 z} \sqrt{1+\frac{2 B_{0 y} v_{x} E_{0}}{c B_{0 z}^{2}}} \approx B_{0 z}+\frac{v_{x}}{c} \frac{B_{0 y}}{B_{0 z}} E_{0}
$$

In particular, if $\overrightarrow{\mathrm{B}_{0}}$ and $\vec{E}$ are not exactly parallel $\left(\mathrm{B}_{0 \mathrm{y}} \neq 0\right)$, then the interaction energy $\vec{\mu} \cdot \vec{B} \overrightarrow{\text { total }}=\mu_{0} \mathrm{gF} \mid B_{\text {total }}$ will change synchronously with reversal of $\vec{E}$, thus mimicking a real atomic EDM. This unfavorable situation is shown in Figure III.24.

\section{The vxE Effect Changes the Magnitude of $B$}

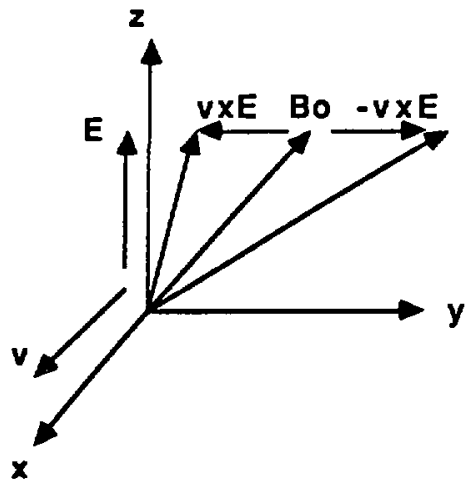

Figure II.24

Even the very small misalignment of $\Theta=1 \mu$ radian will result in an effect comparable to an EDM of $d_{e}=10^{-26} \mathrm{e}-\mathrm{cm}$ ! The problem remains of how to reauce $\Theta$.

In $\$$ III.I.2 we show how the vxE term is separated from the EDM. Here, we demonstrate how to minimize it in the first place. If we write the relationship between the $y$ and $z$ currents and the $y$ and $z$ magnetic fields as

$$
\left(\begin{array}{l}
B_{0 y} \\
B_{0 z}
\end{array}\right)=\left(\begin{array}{ll}
a & b \\
c & d
\end{array}\right)\left(\begin{array}{l}
i_{y} \\
I_{z}
\end{array}\right)
$$

it is clear that for some value of $I_{y}$ and $I_{z}, B_{0 y}$ will vanish. Even though the coefficients 
$a, b, c$, and $d$ change each time the coils moved, we estimate the values basec on the results of a typical run.

We measured the vxE effect when $I_{y}=0$ and found $\Theta_{0}=8.3 \mathrm{mrad}$. Since the $z$-coil has approximately four times as many turns as the $y$-coil, we can write the B-field "rotation" matrix as

$$
\left(\begin{array}{ll}
a & b \\
c & d
\end{array}\right) \approx K\left(\begin{array}{cc}
\cos \Theta_{0} & 4 \sin \Theta_{0} \\
-\sin \Theta_{0} & 4 \cos \Theta_{0}
\end{array}\right) \approx K\left(\begin{array}{cc}
1 . & .04 \\
-.04 & 1 .
\end{array}\right)
$$

where $K$ is some irrelevant constant. Further, we noticed that an applied current of $I_{y}=10$ milliamps rotated $\Theta$ by $5.6 \mathrm{mrad}$ :

$$
\Delta \Theta(\mathrm{mrad}) \approx .56 \Delta \mathrm{I}_{\mathrm{y}}(\mathrm{mamp})
$$

Assuming a target precision of $\sigma_{d}=10^{-26} \mathrm{e}-\mathrm{cm}$ and a vxE reduction factor of 40 (see $\S$ III.I.2), we must guarantee that $\theta=0 \pm 40 \mu \mathrm{rad}$ if we are to trust the experiment. This corresponds to controlling the $y$-current to a level

$$
\mathrm{I}_{\mathrm{y}}=14.8 \pm .07 \text { milliamperes }
$$

and

$$
I_{z}=100 . \pm 2 \text { milliamperes }
$$

because the coefficients $b$ and $c$ are 25 times smaller than $a$ and $d$ Although the exact quantities will depend on the apparatus, this is a good order of magnitude estimate. Both these requirement are easily met by the North Hills constant current supplies that drift no more that $2 \mu$ amps/hour. In addition, we can correct for any drifts by changing $I_{y}$ accordingly. 


\section{PMT Hysteresis}

If the order of the E-field and beam chops were not permuted (see $\S$ IV.A), then there would be a residual systematic originating in long-term gain variations in the phototubes. It is a result of the enormous fluorescent signal that a PMT sees when the beam shutter between it and the nearest oven is open. Under typical operating conditions, when the oven temperature is about $1100^{\circ} \mathrm{K}$ and the cathode-anode voltage on the PMTs is 600 volts, the anode current $I_{\text {anode }}=20 \mu$ amps when the PMT measures the near fluorescence.

The excess of photons floods the PMT's dynodes with electrons. This causes the gain of the tube to be artificially high for about 30 reconds. Because we wait only 2 seconds before taking data, after reversing the atomic beam, the first chops recorded have inflated counts. This gives a syr $\quad 2$ if the same chops are always inflated.

Rather than waiting 30 seconds or more, we solve this problem by varying the crier of the chops. In this way, no single configuration is favored by the hysteresis and the systematic is removed.

Furtt $r$, this effect is independent of th electric field and is demonstrated as negligible by collecting data with $\mathrm{E}=0$ and verifying that the electron $E D M$ is consistent with zero.

\section{Numerical Simulation of Possible Systematic Sources}

We performed numerical simulations of the experiment in order to understand the level of potential systematic effects and their possible interactions with each other. We employed the exact formulae for the two-loop interference signal (III.H.5) and integrated over the $v^{3}$ - Maxwellian velocity distribution of the atomic beam.

We now enumerate these possible sources and give the worst case upper limits that 
we used to determine the ultimate systematic error in the electron EDM. Many of the upper limits are ra. iomly chosen or guessed values of the correct order of magnitude.

An incomple reversal of the electric field (gross overestimate)

$$
\Delta|\vec{E}|=2 \%
$$

Different oven temperatures and, thus, different velocity distributions (realistic guess)

$$
\mathrm{T}_{\text {upper }}-\mathrm{T}_{\text {lower }}=\Delta \mathrm{T}=20^{\circ} \mathrm{K}
$$

The cut-oif of slow aroms in one beam through scattering (educated guess)

$$
v_{\text {min, downgoing }}=1 \times 10^{4} \mathrm{~cm} / \mathrm{sec}
$$

A relative phase error in the if loops (comect order of magnitude, random choices)

$$
\begin{aligned}
& \delta\left(\alpha=+\frac{\pi}{4}\right)=40 \mathrm{mrad}, \delta\left(\alpha=-\frac{\pi}{4}\right)=-20 \mathrm{mrad}, \\
& \delta\left(\alpha=+\frac{3 \pi}{4}\right)=50 \mathrm{mrad}, \delta\left(\alpha=-\frac{3 \pi}{4}\right)=30 \mathrm{nrad}
\end{aligned}
$$

Non-zero and different background signals at each detector (realistic choice)

$$
B_{\text {upper }}=29 \% \text { and } B_{\text {lower }}=32 \%
$$

A non 20 imal if cument in one of the if loops (worst case guess)

$$
\frac{I_{\text {ff.optima] }}-I_{\text {ff,upper }}}{I_{\text {ff.optimei }}}=10 \%
$$

Non-overlapping Rabi patterns (see \& III.H.1) (actual values)

$$
v_{\text {upper }}=-200 \mathrm{~Hz} \text { and } v_{\text {lower }}=200 \mathrm{~Hz}
$$

The Ramsey incerference fringes being situated away from ihe average frequency the Rabi partems (see \& III.E.2 and \& IIIH.H) (worst case guess)

$$
v_{0}=20 \mathrm{~Hz}
$$

An error in the applied ff frequency (worst case quess)

$$
v_{\mathrm{ff}}-\mathrm{v}_{0}=1 \mathrm{~Hz}
$$

\section{A non-zero vxE effect (realistic value)}

$$
\Theta=50 \mu \mathrm{rad}
$$


Using the above values, we found a false electric dipole moment $\mathrm{d}_{e}=1.6 \times 10^{-26} \mathrm{e}-\mathrm{cm}$. This is even an overestimate because the electric field should easily reverse to 1 part in $10^{3}$.

By varying the parameters, we are able to draw several qualitative conclusions from the above study. First, the source of systematics to which we are most sensitive involves the coupling of an incomplete reversal of the electric field, errors in the if phases, and the quadratic Stark effect. This is easy to believe because the four if phases are responsible for removing the Stark effect from the data (see § III.I.2). In fact, if we had used only' two rf phases $\pm \pi / 4$ in conjunction with the above worst case parameters, we would have found an $E D M$ of $d_{e}=1.6 \times 10^{-24} \mathrm{e}-\mathrm{cm}$ !

We also noticed that slight discrepancies in the magneric fields, that give rise to nonoverlapping Rabi patterns and uncentered Ramsey fringes, contribute very little to the systematics. When all other parameter are set to zero, these effect give a false EDM of $\mathrm{d}_{\mathrm{e}}=2.8 \times 10^{-28} \mathrm{e}-\mathrm{cm}$.

We conclude from this investigation that, given the above parameters with the electric field reversibility to 1 part in $10^{3}$, we believe the experiment to be free of systematic effects down to a level of $d_{e}=10^{-27} \mathrm{e}-\mathrm{cm}$. 


\section{K. Noise}

\section{Shot noise}

The ultimate limit on the uncertainty in the electron EDM $\sigma_{d}$ is determined by shot noise, or counting statistics, at the PMT photocathode. The shot noise is

$$
\sigma_{\mathrm{L}_{\mathrm{u}}}=\frac{\hbar \mathrm{h}}{2 \Pi \mathrm{TERL_{ \textrm {E } }}} \sigma(\Delta)
$$

where $\Delta$ is the electric field asymmetry defined in (IIl.I.13). Its uncertainty is

$$
\sigma(\Delta)=q\left[\frac{\left(F_{p e} T\right)(S(+E)-S(-E))}{\left(F_{p e} T\right)(S(+E)+S(-E))}\right]
$$

$\mathrm{F}_{\mathrm{pe}}$ is the flux of photoelectrons (events) and $\mathrm{T}$ is the total integration time. The uncertainty in the ratio is approximately

$$
\sigma(\Delta)=\sqrt{-\frac{1}{\mathrm{~F}_{\mathrm{pe}} \mathrm{T}}}
$$

which is simply $1 / \sqrt{N}$, where the number of events $N=F_{p e} T$. Alrogether, the shot noise in the electric dipole moment of the electron is

$$
\sigma_{\mathrm{d}}=\frac{\hbar v}{2{ }^{2} R E L_{-} \sqrt{F_{p e}} \sqrt{T}}
$$

The flux of photoelectrons is

$$
F_{p e}=\frac{7}{20}\left((A)+\frac{1}{2}(B)\right) \eta F_{d}=\left(.35 x .6 m F_{d}\right.
$$

The factor of $7 / 20$ is the faction on atoms polarized by optical pumping. The second factor involving $A$ and $B$ is the level of the signal on resonance for $\pm \pi / 4$ and $\pm 3 \pi / 4$ relative phase (equations (III.I.10) and (III.I.11)). If we use the following nominal values, 


$$
\begin{gathered}
v=4 \times 10^{4} \mathrm{~cm} / \mathrm{sec} \\
L_{E}=100 \mathrm{~cm} \\
R=-560 \\
E=100 \mathrm{kV} / \mathrm{cm}=330 \text { statvolt } / \mathrm{cm} \\
F_{d}=10^{9} / \mathrm{sec} \\
\eta=.04 \\
\Pi=-.75
\end{gathered}
$$

we find a photoelectron flux $\mathrm{F}_{\mathrm{pe}}=7 \times 10^{6} / \mathrm{sec}$ and a shot noise level:

$$
\sigma_{\mathrm{d}}(\mathrm{e}-\mathrm{cm})=\frac{1 \times 10^{-24}}{\sqrt{\mathrm{T}(\mathrm{sec})}}
$$

Unfortunately, this limit is difficult to achieve because other sources of noise dominate. During our run of 300 minutes, we attained a precision of $3.2 \times 10^{-26} \mathrm{e}-\mathrm{cm}$ (see § IV.B). This corresponds to a noise level of $4 \times 10^{-24} \mathrm{e}-\mathrm{cm} / \sqrt{\mathrm{T}}(\mathrm{sec})$, indicating that the experiment's noise level is currently four times the ultimate shot noise level.

\section{Magnetic Noise}

How much magnetic noise is tolerable, given our technique of chopping and building asymmetries? A reasonable choice is to require that the noise in the signal arising from magnetic field fluctuations be less than the shot noise level.

The sensitivity of the experiment undoubtedly depends on the frequency of the magnetic noise. If $\mathrm{B}(\mathrm{t})$ is the time-varying amplitude of the magnetic noise, then we can construct the Fourier transform $B(\omega)$ :

$$
B(\omega)=\frac{1}{\sqrt{2 \pi}} \int_{-}^{-} B(t) \sin (\omega x) d t
$$


More precisely, in one electric field chop period $E_{\text {period, }}$, the shot noise contributes an uncertainty to an atom's phase

$$
\sigma(u)_{s h o t}=\frac{1}{2 \Pi \sqrt{F_{p e} T}}
$$

and the magnetic noise at a frequency $\omega_{\mathrm{E}}=2 \pi / \mathrm{E}_{\text {period }}$ causes an error in the phase of

$$
\sigma(u)_{\text {mag }}=\frac{\mu_{0} g_{F} L_{x f}}{2 \Pi v} B\left(\omega_{E}\right)
$$

Demanding that

$$
\sigma(\mathrm{u})_{\text {mag }}<\sigma(\mathrm{u})_{\text {shot }}
$$

we establish a condition on $B\left(\omega=\omega_{E}\right)$ :

$$
B\left(\omega=\omega_{E}\right)<\frac{v}{\mu_{0} g_{F} L_{\text {Tf }} \sqrt{F_{p e} T}} .
$$

This is the worst case because chopping the E-field at exactly the same frequency as the noise leads to no attenuation. A close analysis of other frequencies gives the expected $\zeta=\frac{\omega_{E}}{\omega_{B}}$ attenuation for low frequencies $\omega_{B}$ and an attenuation of $\zeta \approx \frac{\omega_{B} \tau}{2 \pi}=\frac{\omega_{B} L_{x f}}{2 \pi v}$ for high frequencies. Physically, a low frequency magnetic field will be chopped away by the E-field chop. A fluctuation with frequency higher than 1/ (transit time of an atom between the rf loops) will be averaged over and, thus, reduced. We summarize our requirements on the amplitude of magnetic noise at a frequency $\omega$ :

$$
\text { where } \zeta=\left\{\begin{array}{c}
\frac{v}{\omega} \text { for } \omega<\omega_{E} \\
1 \text { for } \omega \approx \omega_{E} \\
\frac{\omega L_{r} \text { for } \omega>\omega_{E}}{2 \pi v}
\end{array} \mid\right.
$$

Figure III.25 shows the maximum allowable $B(\omega)$ in the region of the atomic beam when the electric field is reversed at a rate of $1 \mathrm{~Hz}$. 


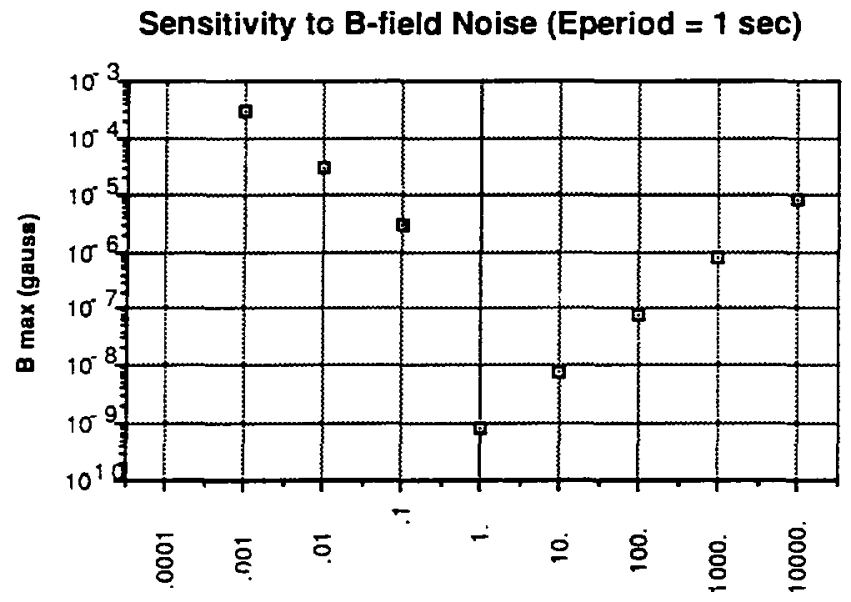

Frequency of Megnetle Nolse $(\mathrm{Hz})$

Figure III.25

It is very difficult to characterize accurately the level of magnetic noise in the laboratory. However, by measuring the induced voltage in a large rectangular coil, we inferred the amplitude of ambient time-varying magnetic fields through Faraday's Law. Because we measured no induced voltages to a cerain precision, we were able to establish upper limits on the size of the noise. The results are summarized in the following table:

frequency $\omega / 2 \pi(\mathrm{Hz})$

1

10

60

1000 upper limit on $B(\omega)$ (gauss)

.060

.006

.001

.0006

We conclude that a shielding factor of $2 \times 10^{5}$ is probably necessary if we are to reach our desired ultimate precision. 


\section{ADC Noise}

Just as we required that the magnetic noise to be no larger than the shot noise, we also desire the analog to digital converters to work no better or no worse. Because we digitize the signal once every 1 millisecond, the ADC's must have a precision better than the shot noise in 1 millisecond, which is roughly $8 \times 10^{-3}$ (using the parameter in \$III.K.1). This easily satisfied by the 12-bit ADC's which have a precision of 1 bit in $10^{12}=2 \times 10^{-4}$. In fact, we could even digitize the signal once every 100 milliseconds without any noticeable increase in noise.

\section{Phototube Dark Noise}

The EMI 9658B phototubes have a typical dark current of $1 \mathrm{nA}$ and a maximum dark current of $I_{\text {dark, max }}=10 \mathrm{nA}$ when the gain of the tube is $10^{6}$. The maximum dark current corresponds to $n_{\text {dark }}=6 \times 10^{4}$ counts $/ \mathrm{sec}$ at the photocathode. In order to neglect the $1 / \sqrt{ } \mathrm{N}$ fluctuations in the tube's dark noise, we demand that $\sqrt{ }_{\text {dark }}<\sqrt{F_{p e}}$. This is easily satisfied.

\section{Scattered Atoms and Laser Power Fluctuations}

We believe that the two limiting sources of noise in the current experiment are atomic beam intensity variations and fluorescence from scattered atoms. Scattered atoms interact with the unsaturated, low intensity regions of the laser beam and, therefore, couple in any noise in the laser power. The laser power fluctuations are quite large - typically $2.5 \%$ RMS into $10 \mathrm{~Hz}$ to $100 \mathrm{kHz}$. This amount of noise could easily cause problems. 
Evidence for this unwanted fluorescent background is the height of the pumping dip in Figure III.9. The exact cause of scattering is unclear, but, in past versions of the apparatus, additional collimating slits, located on each side of the fluorescent detector ellipsoids, considerably reduced the level of the optical pumping dip. Plans are underway to add slits to reduce this background.

\section{Atomic Beam Fluctuations}

The other limiting source of noise is the unpredictable variations in the flux of atoms produced by the ovens. Time constants for these fluctuations are on the order of seconds and the net flux changes are sometimes as large as $30 \%$. We believe that the ovens undergo periods of instability during which the data are very noisy. Further study of this problem is warranted. 


\section{Results and Conclusions}

\section{A. The Data Collection Cycle}

We now discuss the exact manner in which the data were collected during the successiul twenty-four hour run. The data collection sequence is completely defined once the following variable parameters are set.

The first set of parameters are the periods: $R F_{\text {period }}, E_{\text {period, }}$ and $B_{\text {period. }}$ Each period is the length of time required for its respective chop to cover all possible values. For example, if $R F_{\text {period }}=400$ milliseconds, each relative phase $\alpha$ lasts for 100 milliseconds because there are four possible if phases $\pm \pi / 4$ and $\pm 3 \pi / 4$. If the beam period is $B_{\text {period }}=25600$ milliseconds, the computer reverses the atomic beam direction once every 12.8 seconds. In all cases $R F_{\text {period }}<\mathrm{E}_{\text {period }}<\mathrm{B}_{\text {period }}$ and, in addition, each period must divide into the longer periods an even number of times. In our 24 hour run, we used the following periods: $R F_{\text {period }}=400 \mathrm{msec}, \mathrm{E}_{\text {period }}=1600 \mathrm{msec}$, and $B_{\text {period }}$ $=25600 \mathrm{msec}$.

The next set of parameters are the delays. A delay is the period of time that the computer waits before collecting data each time a particular chop is changed. The $\mathrm{RF}_{\text {delay }}$ $=20 \mathrm{msec}$ is necessary because it takes an atom several milliseconds to traverse the appararus. We use an $\mathrm{E}_{\text {delay }}=200$ msec to ensure that the eiectric field plates have more than enough time to charge when they are reversed. Finally, the $B_{\text {delay }}=2000 \mathrm{msec}$ allows the beam shutter enough time to complete its relatively slow, mechanical movement. It also helps reduce the phototube hysteresis, although hysteresis is principally corrected by permuting the order of the chops.

A sweep is a complete run through all of the possible chops in a single beam period. 
Thus, if all the delays are zero, a sweep takes the same amount of time as a beam period, the longest of the periods. However, with non-zero delays, a sweep takes a total time

$$
T_{\text {sweep }}=B_{\text {period }}+2 B_{\text {delay }}+\frac{2 B_{\text {period }}}{E_{\text {period }}} E_{\text {delay }}+\frac{4 B_{\text {period }}}{R F_{\text {period }}} R F_{\text {delay }}
$$

We can define a data collection efficiency $\varepsilon$

$$
\varepsilon=\frac{B_{\text {period }}}{T_{\text {sweep }}}
$$

With the periods and delays defined above, we find an efficiency of $\varepsilon=62 \%$. This is equivalent to saying that if we collect data for 1 day (real time), we will obtain .62 day of data (integration time) assuming no time is wasted between cycles.

A cycle consists of five sweeps, the first of which is the calibration sweep for the analyzing powers (see § III.I.3). The next four sweeps begin with different orderings of the E-field cinip and beam direction chop. Because each of the two chops has two directions, there are four different possible starting configurations. Figure IV.1 depicts the initial chop in each of the five sweeps in a given cycle.

$\begin{array}{ccc}\text { sweep \# } & \text { initial E-field } & \text { initial beam direction } \\ 1 & \text { none } & \Downarrow \\ 2 & + & \Uparrow \\ 3 & + & \Downarrow \\ 4 & - & \Uparrow \\ 5 & - & \Downarrow\end{array}$

Figure IV.1

This permutation of the initial $\mathrm{E}$ and beam chops equalizes the effects of PMT hysteresis between the two electric field directions: a negative electric field is the first chop twice and positive electric field is the first chop twice. 
With the above defined periods and delays, it takes $5 \times \mathrm{T}_{\text {sweep }}=206$ seconds $=3.4$ real minutes to complete one cycle. A cycle, however, represents only $4 \times 25.6$ seconds = 1.71 minutes of integration time because the first sweep is spent calibrating the analyzing powers. Each time a cycle is completed, the data are stored on the computer's disk and the optical frequency and if frequency are stabilized. Henceforth, the cycle is the elementary unit of data.

\section{B. The Data and Cuts}

We collected our data over a period of 24 hours, during which we recorded a total of 210 cycles. This is equivalent to 358 minutes, or roughly 6 hours of integration time. With an efficiency of $62 \%$, this task should have taken less than 10 hours. Laser stabilization, rf stabilization, and manually adjusting the beam shurters accounts for the rest of the time.

A recurring problem throughout the run was the mechanical slipping of the atomic beam shutters. These shutters are responsible for blocking and opening each beam. When a shutter failed, it was immediately apparent in that the analyzing power was zero or anomalously low in absolute value. Because the analyzing power is measured in the first sweep of a given cycle, it is possible that a shutter failed during sweeps $2-5$. This would suggest that we should not only reject a cycle with an anomalously low analyzing power, but also the cycle directly preceding it. This extra cut was tried and the resulting average and standard deviation of the distribution remained relatively unchanged. 
Thus, the only cut that we apply to the data is that we require the absolute value of each analyzing power to be greater than 0.55 . This cut leads us to include 177 cycles and reject 33, yielding a total usable integration time $T=302$ minutes. The resulting electron electric dipole moment is

$$
d_{e}=0.1 \pm 3.2 \times 10^{-26} \mathrm{e}-\mathrm{cm}
$$

Figure IV.1 is a histogram displaying the number of measurements (cycles) that lie within a $\pm 5 \times 10^{-26} \mathrm{e}-\mathrm{cm}$ window of the indicated center EDM.

\section{Histogram of EDM Data}

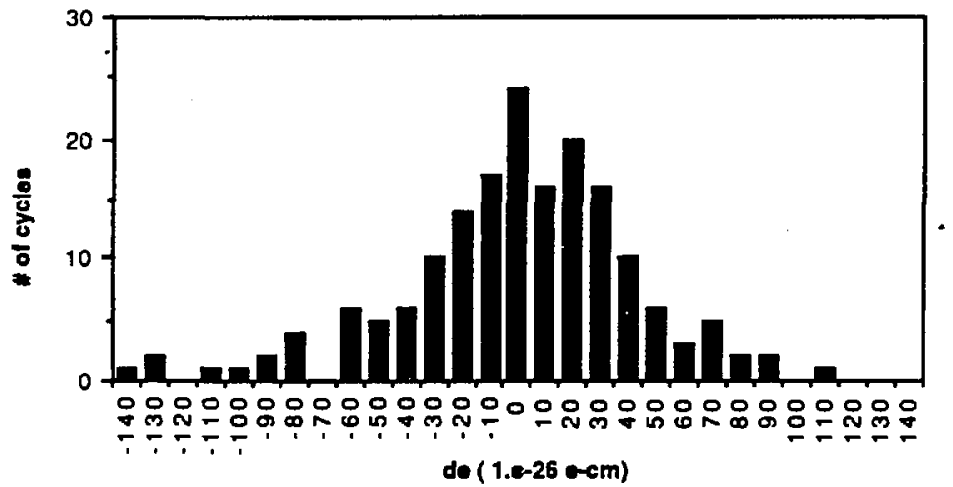

Figure IV.1

The histogram indicates that the data are normally distributed, just as they should be in the absence of systematic effects. The FWHM of the distribution is roughly $50 \times 10^{-26}$ e-cm. This corresponds to the typical error per cycle. When this full-width is divided by 
the square root of the number of included events $\sqrt{177}$, the resulting uncertainty in the mean is $3.8 \times 10^{-26}$, which agrees rather well with the uncertainty obtained by directly analyzing the distribution of cycles.

\section{Concluding Remarks}

This measurement of $d_{e}$ constitutes a new upper limit. Although it is not precise enough to influence the more realistic theoretical models, it represents the first step in a technique that promises a factor of ten or even one hundred improvement in accuracy.

This new upper limit on $d_{e}$ does affect two models that we carlier considered: the "non-minimal" Standard Model with heavy neutrinos and the left-right symmetric model (see § II.B.2). In the "non-minimal" Standard Model, a new limit on the masses of the unseen neutrinos can be inferred. Our measurement places a new upper bound on the mass of the right-handed neutrino in the left-right symmetric model. The new upper limit on the electron EDM also matches the current limit on models with horizsntal interactions.

There are many changes that almost certainly would improve the experiment's precision. We have already mentioned several, but we will recapitulate.

The implementation of additional collimating slits on each side of the fluorescent detector ellipsoids will reduce the scartered atom fluorescent background. This will both increase the analyzing powers and reduce the noise.

An improved electric field switch has already been designed and built. It is hoped that this new unit will enable the experiment to operate at electric fields as $E=150 \mathrm{kV} / \mathrm{cm}$ and, accordingly, increase the ultimate precision by $150 \%$ over the $E=100 \mathrm{kV} / \mathrm{cm}$ run. 
Any improvement in the main chamber pressure would increase the flux of atoms, thereby reducing the potential shot noise limit.

The experiment would benefit greatly if the fluctuations in the atomic beam intensity are removed or normalized. This problem definitely warrants further study and development.

Once the noise has been reduced successfully and the experiment's precision is well into the $10^{-27} \mathrm{e}-\mathrm{cm}$ regime, it will probably be necessary to further remove any possible systematics. Velocity selection of the atomic beam with chopper wheels might be a valuable addition to the experiment

Finally, this EDM experiment will never reach a level of precision that could influence the standard model unless vast changes are made. One very speculative possibility is the use of laser-cooling to slow the atoms in the atomic beam. Although there are problems with the total flux of cooled atoms, slower atoms could significantly reduce the linewidth of the Ramsey pattem and, therefore, yield a very precise result. 


\section{Appendix A}

\section{The $C_{9}$ P, and T Symmetries of an Electron EDM}

In this appendix, we present a manifestly Lorentz invariant discussion of the $C, P$, and $T$ transformation properties of a perturbing term in the Dirac Lagrangian corresponding to an electric dipole moment. First, we examine the transformation properties of the electromagnetic field strength tensor $F^{\mu \nu}$. Next, we show how Dirac fields transform, and finally consider the perturbing $L E D M$.

The electromagnetic field strength tensor transforms as

$$
\begin{array}{ccc}
\mathrm{F}^{\mu v}=\partial^{\mu} \mathrm{A}^{v}-\partial^{v} \mathrm{~A}^{\mu} \\
\mathrm{P} \partial^{\mu} \mathrm{P}^{-1}=\mathrm{g}_{\mu \mu} \partial^{\mu} & \text { and } & \mathrm{PA}^{v} \mathrm{P}^{-1}=\mathrm{g}_{\mathrm{vv}} \mathrm{A}^{v} \\
\mathrm{C} \partial^{\mu} C^{-1}=\partial^{\mu} & \text { and } & \mathrm{CA}^{v} C^{-1}=-A^{v} \\
\mathrm{~T} \partial^{\mu} \mathrm{T}^{-1}=-\mathrm{g}_{\mu \mu} \partial^{\mu} & \text { and } & \mathrm{T} \mathrm{A} \mathrm{A}^{v} \mathrm{~T}^{-1}=\mathrm{g}_{v v} A^{v}
\end{array}
$$

yielding the transformation properties of $F^{\mu v}$ :

$$
\begin{aligned}
& P F^{\mu v} P^{-1}=g_{\mu \mu} g_{v v} F^{\mu v} \\
& C F^{\mu v} C^{-1}=r-F^{\mu v} \\
& T F^{\mu v} T^{-1}=-g_{\mu \mu} g_{v v} F^{\mu v}
\end{aligned}
$$

A consistent procedure for a Dirac field $\Psi$ would be ${ }^{37}$

$$
\begin{gathered}
P \psi(\vec{x}, t) P^{-1}=\gamma_{0} \psi(\vec{x}, t) \\
C \psi(\vec{x}, t) C^{-1}=i \gamma_{0} \gamma_{2} \psi(\vec{x}, t) \\
T \psi(\vec{x}, t) T^{-1}=i \gamma_{1} \gamma_{3} \psi(\vec{x},-t)
\end{gathered}
$$

LEDM is odd under parity

$$
\begin{aligned}
P i_{E D M} \mathrm{P}^{-1} & =\mathrm{P} \bar{\psi} \mathrm{i} \gamma_{S} \sigma_{\mu v} \mathrm{~F}^{\mu v} \psi \mathrm{P}^{-1}=\mathrm{i} \bar{\psi} \gamma_{0} \gamma_{S} \sigma_{\mu v} \gamma_{0} \psi \mathrm{g}_{\mu \mu} \mathrm{g}_{v v} \mathrm{~F}^{\mu v} \\
& =-i \mathrm{~g}_{\mu \mu} \mathrm{g}_{w} \bar{\psi} \gamma_{5} \gamma_{0} \sigma_{\mu v} \gamma_{0} \psi \mathrm{F}^{\mu v}
\end{aligned}
$$

37 M. Suzuki, Physics 225B lecture notes, UC Berkeley. 
by manipulatirig the Dirac matrices, we find that

$$
\gamma_{0} \gamma_{\mu} \gamma_{v} \gamma_{0}=g_{\mu \mu} g_{v v} \gamma_{\mu} \gamma_{v}
$$

Inserting this, we see that

$$
\mathrm{P} L_{E D M} \mathrm{P}^{-1}=-\mathrm{i} \bar{\psi} \gamma_{5} \sigma_{\mu v} \psi \mathrm{F}^{\mu v}=-L_{E D M}
$$

We now show that $L E D M$ is odd under time-reversal

$$
\begin{gathered}
\mathrm{T} L_{E D M} \mathrm{~T}^{-1}=\mathrm{T} \bar{\psi} \mathrm{i} \gamma_{5} \sigma_{\mu v} \mathrm{~F}^{\mu v} \psi \mathrm{T}^{-1} \\
=\bar{\psi}(-\mathrm{i}) \gamma_{3} \gamma_{1}(-\mathrm{i}) \gamma_{5} \sigma_{\mu v}^{*} \mathrm{i} \gamma_{1} \gamma_{3} \psi\left(-\mathrm{g}_{\mu \mu} \mathrm{g}_{v v} \mathrm{~F}^{\mu v}\right) \\
=\bar{\psi} \mathrm{i} \gamma_{5}\left(\gamma_{3} \gamma_{1} \sigma_{\mu v}^{*} \gamma_{1} \gamma_{3}\right) \psi\left(-\mathrm{g}_{\mu \mu} \mathrm{g}_{v v} \mathrm{~F}^{\mu v}\right) \\
\bar{\psi} \mathrm{i} \gamma_{5}\left(\mathrm{~g}_{\mu \mu} \mathrm{g}_{v \nu} \sigma_{\mu v}\right) \psi\left(-\mathrm{g}_{\mu \mu} \mathrm{g}_{v v} \mathrm{~F}^{\mu v}\right) \\
=-L_{E D M}
\end{gathered}
$$

And that $\angle E D M$ is even under charge coniugation

$$
\begin{gathered}
C L_{E D M} C^{-1}=\psi^{T}(-i) \gamma_{2} \gamma_{0} i \gamma_{S} \sigma_{\mu v} \text { i } \gamma_{0} \gamma_{2} \bar{\psi}^{T}(-1) F^{\mu v} \\
=\psi^{T}(-i) \gamma_{5}\left(\gamma_{2} \gamma_{0} \sigma_{\mu v} \gamma_{0} \gamma_{2}\right) \bar{\psi}^{T} F^{\mu v} \\
=\psi^{T}(-i) \gamma_{S}\left(-\sigma_{\mu v}^{T}\right) \bar{\psi}^{T} F^{\mu v} \\
=+L_{E D M}
\end{gathered}
$$




\section{Appendix B}

\section{A Calculation of the EDM in a Theory with Horizontal Couplings}

Here we consider a leptoquark coupling of the form

$$
\begin{aligned}
L & =\lambda_{R} \bar{q}_{R} e_{L}+\lambda_{L} \bar{q}_{L} e_{R}+\text { h.c. } \\
& =\frac{\lambda_{R}}{2} \bar{q}\left(1+\gamma_{S}\right) e+\frac{\lambda_{L}}{2} \bar{q}\left(1-\gamma_{S}\right) e+\text { h.c. }
\end{aligned}
$$

which leads to a Feynman diagram:

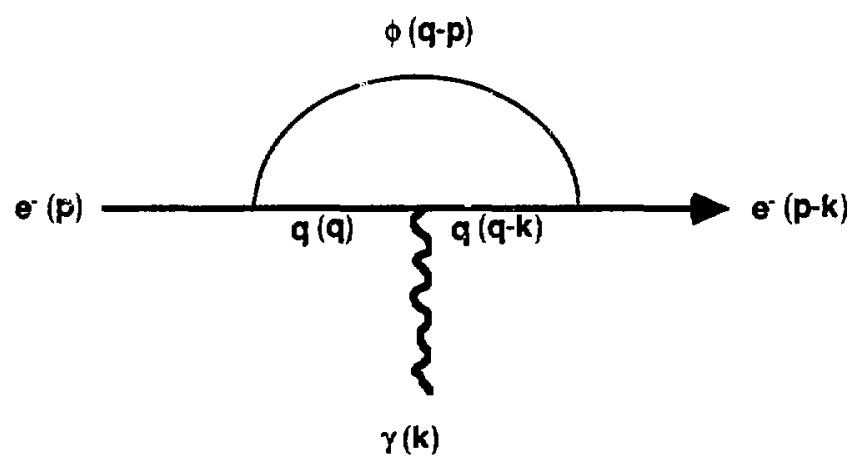

Scalar leptoquark contribution
to electron EDM

Figure B.1

If the outgoing electron is right handed, the matrix element is 


$$
M_{R}=\frac{\mathrm{Q} \lambda_{R}^{*} \lambda_{\mathrm{L}}}{4(2 \pi)^{4}} \int_{0}^{-} \frac{\mathrm{d}^{4} \mathrm{q}}{(\mathrm{q}-\mathrm{p})^{2}-\mathrm{m}_{\mathrm{0}}^{2}} \overline{\mathrm{u}}(\mathrm{p})\left(1+\gamma_{\mathrm{s}}\right) \frac{1}{\mathrm{q}-\mathrm{k}^{\mathrm{k}}-\mathrm{m}_{\mathrm{q}}} \frac{1}{\mathrm{q}-\mathrm{m}_{\mathrm{q}}}\left(1+\gamma_{5}\right) \mathrm{u}(\mathrm{p}-\mathrm{k})
$$

where $Q=2 / 3$ is the quark charge. We can rewrite the Dirac part of (B.2) as

$$
\begin{aligned}
& \overline{\mathrm{u}} \ldots \mathrm{u}=\frac{1}{\left[(\mathrm{q}-\mathrm{k})^{2}-\mathrm{m}_{\mathrm{q}}^{2}\right]\left[\mathrm{q}^{2}-\mathrm{m}_{\mathrm{q}}^{2}\right]} \overline{\mathrm{u}}\left(\left(1+\gamma_{5}\right) .\right. \\
& {\left[d x+m_{q} q t-k t k-m_{q} k t+m_{q} e d+m_{q}^{2} d\right]\left(1+\gamma_{5}\right) u}
\end{aligned}
$$

Because the product of an odd number of gamma matrices sandwiched between two righthanded operators vanishes, we are left with:

$$
\overline{\mathrm{u}} \ldots \mathrm{u}=\frac{1}{\left[(\mathrm{q}-\mathrm{k})^{2}-\mathrm{m}_{\mathrm{q}}^{2}\right]\left[\mathrm{q}^{2}-\mathrm{m}_{\mathrm{q}}^{2}\right]} \overline{\mathrm{u}}\left(\left(1+\gamma_{5}\right) \mathrm{m}_{\mathrm{q}}[\mathrm{q} t-k t+\alpha \dot{q}]\left(1+\gamma_{5}\right) \mathrm{u}\right.
$$

Utilizing the anti-commutation rules for gamma matices, we obtain

$$
\bar{u} \ldots u=\frac{1}{\left[(q-k)^{2}-m_{q}^{2}\right]\left[q^{2}-m_{q}^{2}\right]} \bar{u}\left(\left(1+\gamma_{5}\right) m_{q}\left[-m_{q} \gamma_{\mu} \gamma_{v} k^{\mu} \varepsilon^{v}+\varepsilon \cdot q\right]\left(1+\gamma_{5}\right) u\right.
$$

We now extract the familiar CP violating part and put it back into the matrix element (B.2) as follows:

$$
M_{C P \text { odd }}^{R}=\frac{-\mathrm{Q} \mathrm{m}_{\mathrm{q}} \lambda_{\mathrm{R}}^{*} \lambda_{\mathrm{L}}}{2(2 \pi)^{4}} \int_{0}^{-} \frac{\mathrm{d}^{4} \mathrm{q} \mathrm{u}(\mathrm{p}) \gamma_{5} \gamma_{\mu} \gamma_{V} \mathrm{k}^{\mu_{\varepsilon}} \mathrm{u}(\mathrm{p}-\mathrm{k})}{\left[(\mathrm{q}-\mathrm{p})^{2}-\mathrm{m}_{\mathrm{Q}}^{2}\right]\left[(\mathrm{q}-\mathrm{k})^{2}-\mathrm{m}_{\mathrm{q}}^{2}\right]\left[\mathrm{q}^{2}-\mathrm{m}_{\mathrm{q}}^{2}\right]}
$$

and we do the same thing for a left handed matrix element. When the two are added, the resulting expression has a real part:

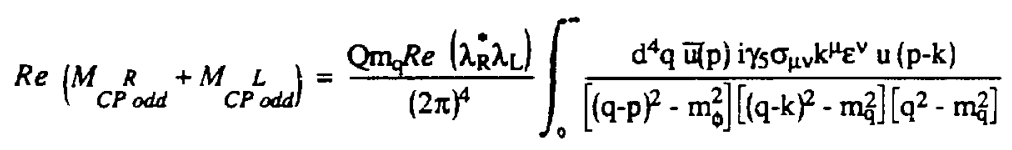

In the limit $k \rightarrow 0$ and $p \rightarrow 0$. we obtain the static EDM:

$$
\mathrm{d}_{e}=\frac{\mathrm{G} \mathrm{m}_{\mathrm{q}} \operatorname{Re}\left(\lambda_{\mathrm{R}}^{*} \lambda_{\mathrm{L}}\right)}{(2 \pi)^{4}} \int_{0} \frac{\mathrm{d}^{4} \mathrm{q}}{\left[\mathrm{q}^{2}-\mathrm{m}_{\phi}^{2}\right]\left[\mathrm{q}^{2}-\mathrm{m}_{\mathrm{q}}^{2}\right]^{2}}
$$

To evaluate the integral 


$$
I\left(q^{2}\right)=\int_{0} \frac{d^{4} q}{\left[q^{2}-m_{\phi}^{2}\right]\left[q^{2}-m_{q}^{2}\right]^{2}}
$$

we first do a Feynman parameterization:

$$
I\left(q^{2}\right)=2 \int_{0}^{-} d^{4} q \int_{0}^{1} d \alpha \int_{0}^{1-\alpha} d \beta \frac{1}{\left[q^{2}-(\alpha+\beta)\left(m_{q}^{2}-m_{\phi}^{2}\right)-m_{\phi}^{2}\right]^{3}}
$$

which can be rewritten

$$
I\left(q^{2}\right)=2 \pi^{2} \int_{0}^{a} d\left(q^{2}\right) q^{2} \int_{0}^{1} d \alpha \int_{0}^{-a} d \beta \frac{1}{\left[q^{2}-(\alpha+\beta)\left(m_{q}^{2}-m_{\phi}^{2}\right)-m_{\phi}^{2}\right]^{3}}
$$

Performing the integrals over $\alpha$ and $\beta$ and making a Wick rotation such that $q_{0} \rightarrow$ iq0, we find

$$
I\left(q^{2}\right)=\frac{\pi^{2}}{M} \int_{0}^{-} d x \times\left[\frac{1}{\left(x+M+m_{\phi}^{2}\right)^{2}}+\frac{1}{M\left(x+M+m_{\phi}^{2}\right)}-\frac{1}{M\left(x+m_{\phi}^{2}\right)}\right]
$$

where $M=m_{q}^{2}-m_{\odot}^{2}$ and $x$ corresponds to $q^{2}$. This is then integrated to give

$$
f(x)=\frac{-x}{x+M+m_{\phi}^{2}}+\ln \left(x+M+m_{\phi}^{2}\right)+\frac{m_{\phi}^{2}}{M} \ln \left(x+m_{\phi}^{2}\right)-\frac{m_{\varphi}^{2}}{M} \ln \left(x+M+m_{\phi}^{2}\right)-1
$$

Thus I becomes

$$
I=f(\infty)-f(0)=\frac{-\pi^{2}}{m_{q}^{2}-m_{\phi}^{2}}\left(1+\ln \left(\frac{m_{q}^{2}}{m_{\phi}^{2}}\right)\right)=\frac{\pi^{2}}{m_{\phi}^{2}}\left(1+\ln \left(\frac{m_{q}^{2}}{m_{\phi}^{2}}\right)\right)
$$

which finally gives an electron EDM of

$$
d_{e}=\frac{Q R e\left(\lambda_{R}^{*} \lambda_{L}\right)}{16 \pi^{2}} \frac{m_{q}}{m_{\oplus}^{2}}\left(1+\ln \left(\frac{m_{q}^{2}}{m_{\oplus}^{2}}\right)\right)
$$




\section{Appendix C}

\section{A Model Ramsey Magnetic Resonance Experiment}

As an exercise we review the essential features of a "classical" Ramsey magnetic resonance experiment designed to look for an atomic EDM. The arguments presented in this heuristic picture will be classical rather than quantum mechanical. Although our experiment differs somewhat from this simplified model, it will, nevertheless, give the reader an immediate physical picture. A schematic of the experiment is given below in figure C.1.

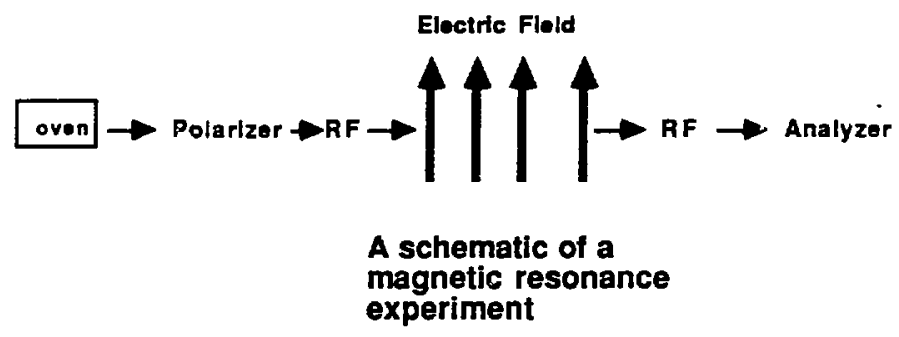

Figure C.1

The entire apparatus sits in an applied, weak magnetic field $\overrightarrow{\mathrm{B}_{0}}$ along the $\mathrm{z}$-axis. The atomic oven boils off atoms to produce a beam of atoms. The polarizer drives the atoms into the $\mathrm{mF}=+1$ state $\left(\vec{F}\right.$ parallel to $\left.\overrightarrow{B_{0}}\right)$. Each $r f$ region contains a magnetic field $\vec{B}_{1} \cos \omega_{0} t$. If we leave the lab frame and work in a rotating frame, these fields are stationary ( say $\vec{B}_{1} \| x$-axis). The first if induces a "flop-in" transition via the torque $\vec{\tau}=\vec{\mu} \times \vec{B}$. Given the right if power, this leaves $\vec{F}$ in the $x-y$ plane (See the figure below). Now, here is the key. If there is no additional energy shift, $\vec{F}$ will remain fixed as the atom travels through the electric field. However, if there is any type of interacrion (say $\overrightarrow{d_{\mathbf{t a m}}} \cdot \vec{E}$ ), $\vec{F}$ will precess about the $z$-axis. If this happens, the atom will 
experience an incomplete "flop-out" at the second if (Remember, the second if also has $\vec{B}_{1} \| x$-axis if the two $f$ 's are in phase). The analyzer measures the angle between $\vec{F}$ and $\overrightarrow{B_{0}}$. If it is not 180 degrees, then there was an energy shift, suggesting a nonzero atomic EDM.
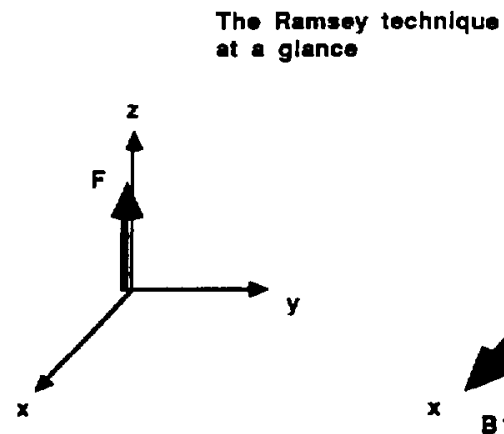

(after polarizer, botore 1st it)
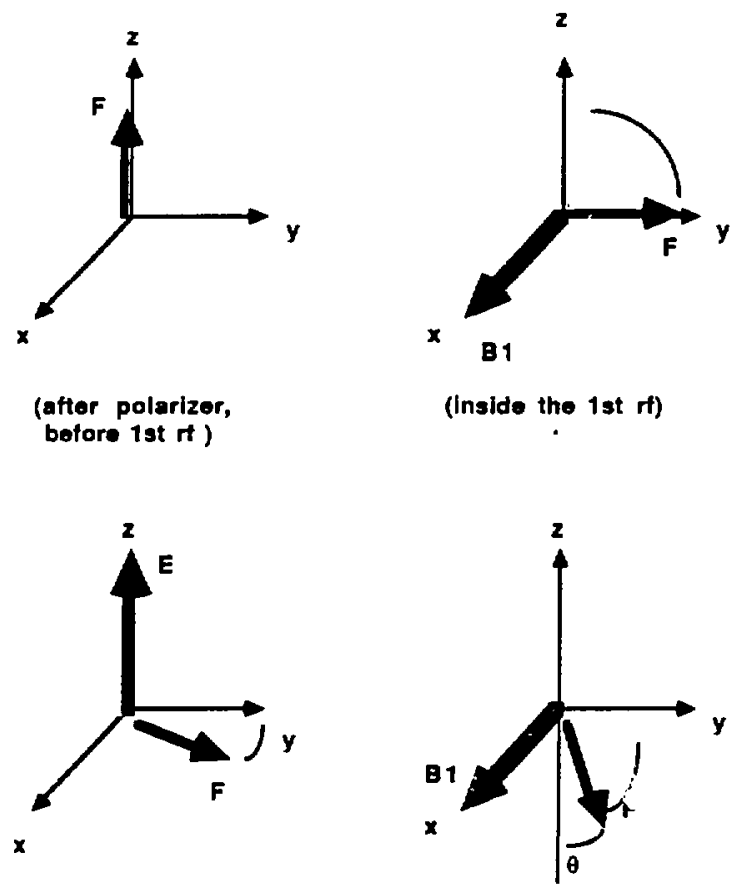
(F precessing due to atomic EDM)

(about to leave 2nd it...incomplete "flopout" due to EDM)

Figure C.2 


\section{Appendix D}

\section{The Low Lying Energy Levels of Thallium}

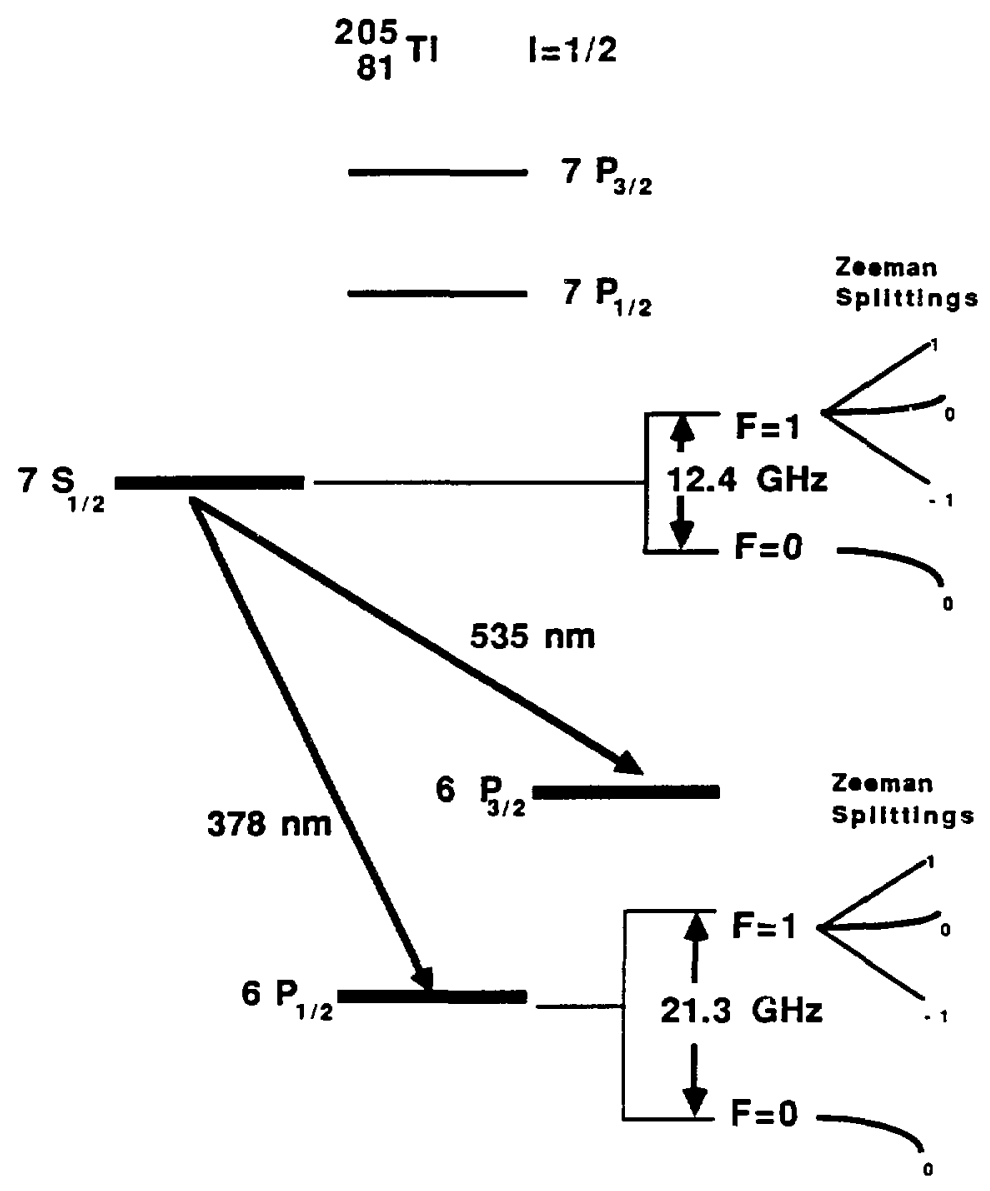




\section{Appendix E- The Lande g-factor of the $\mathrm{Tl}^{205}$ ${ }^{2} \mathrm{P}_{1 / 2}(\mathrm{~F}=1)$ State}

The hyperfine state $\mathrm{g}_{\mathrm{F}}$ factor used in magnetic dipole interactions is calculated from the corresponding elecrionic and auclear Lande factors in the following manner. The expectation value of the atomic magnetic dipole moment in a given hyperfine state is

$$
\left\langle F m_{F}|\mu| F m_{F}\right\rangle=\frac{g_{F} \mu_{0}}{F(F+1)}\left\{g g \frac{F(F+1)+J(J+1)-I(I+i)}{2}+g_{I} \frac{F(F+1)+J(J+1)-I(I+1)}{2}\right\}
$$

We are primarily interested in the $6^{2} \mathrm{P}_{1 / 2}\left(\mathrm{~F}=1\right.$ ) state which has the values: $\mathrm{g}_{\mathrm{J}}=2 / 3, \mathrm{~F}=$ $1, \mathrm{~J}=1 / 2, \mathrm{I}=1 / 2$. We neglect the contribution of the nucleus because

$$
\left|\mu_{n}\right| \approx \frac{\mid d \hbar}{2 m_{n} c} \ll \frac{|e| \hbar}{2 m_{e} c}=\mu_{e}
$$

These values yield a hyperfine Lande-g factor of

$$
g_{F}\left(\mathrm{Tl} 6 \mathrm{P}_{1 / 2}, \mathrm{~F}=1\right) \approx 1 / 3 \text {. }
$$




\section{Appendix F}

\section{The Computer and Data Collection Electronics}

A Compaq 286 (a $12 \mathrm{MHz}$ IBM AT clone) controls the experiment through 3 - 12 bit DAC's, 8 - 12 bit ADC's, 40 - digital I/O lines, and a IEEE-488 (GPIB) communication bus (Metrabyte card\#'s DAS-20, DDA-06, and IE-488). The computer is equipped with a 80287 math coprocessor $(8 \mathrm{MHz})$ and a Sony Trinitron monitor.

The computer chops the if phase, the electric field direction, and the atomic beam direction. In addition, it stabilizes the frequencies of the optical and if transitions.

For each of the two detectors, the output of the PMT is connected to a current-tovoltage converting preamp which is remotely swirched between low gain (1 Volt $/ \mu \mathrm{amp}$ ) and high gain ( 25 Volts $/ \mu \mathrm{amp}$ ) in order to accommodate the reversing atomic beams. The preamp, acting as a voltage source, drives a signal into an Evan's $4130 \mathrm{~A}$ gated integrator that integrates for $900 \mu \mathrm{sec}$ out of each msec. Finally, every $1 \mathrm{msec}$, the computer reads and digitizes the voltage at the output of the gated integrator and resets it. The data is sorted by chop and stored on the computer's 40 Mbyte hard disk. The system is shown schematically in Figure F.1. 


\section{The Detection Electronics}

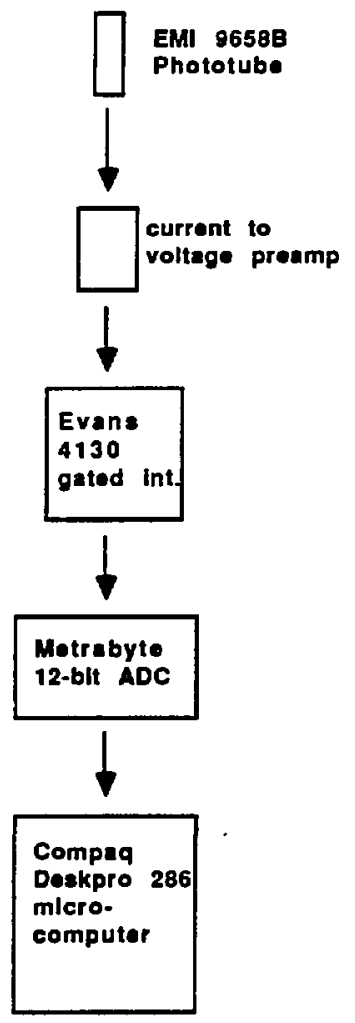

Figure F.1 


\section{A Glossary of Symbols and Constants}

$\alpha$

$\alpha_{0}$

$\overrightarrow{\mathrm{B}_{0}}$

$\overrightarrow{B_{1}}$

$\mathrm{d}_{\mathrm{e}}$

$\vec{E}$

$\eta$

$\mathrm{F}_{0}$

$F_{d}$

$F_{\text {pe }}$

$\gamma$

$\mathrm{L}_{\mathrm{E}}$

LIf

$\mathrm{L}_{\mathrm{far}}$

$\lambda$

$\mathbf{R}$

$R(\alpha, \theta, \eta)$

$\Theta$

$\overline{\mathrm{v}_{\mathrm{o}}}$

$\overline{\mathbf{v}}$ most probable velocity in beam

most probable velocity in oven $=3 \times 10^{4} \mathrm{~cm} / \mathrm{sec}$ for $\mathrm{T}=1100 \mathrm{~K}$

static magnetic field in $\mathrm{z}$-direction $=.28$ gauss

magnetic field of RF transiton

electric dipole moment of the electron (e-cm units)

electric field $=100 \mathrm{kV} / \mathrm{cm}$ (usually)

fluorescent detector efficiency $=4 \%$

total flux of atoms leaving oven slit

flux of atoms reaching the far detector

flux of photoelectrons in the far photoube

$\mu_{0 g}$

length of electric field plates $=100 \mathrm{~cm}$

distance between the two RF loops $=130 \mathrm{~cm}$

distance between oven slit and the far collimating slit $=210 \mathrm{~cm}$

mean free path

enhancement factor of $\mathrm{Tl}=-560 \pm 50$

operator for M1 transiton in a if loop

misalignment angle of $\vec{E}$ and $\overrightarrow{B_{0}}$ giving rise to vxE effect

average velocity of an atom in the oven

average velocity of an atom in the atomic beam $=4 \times 10^{4} \mathrm{~cm} / \mathrm{sec}$

for $\mathrm{T}_{\text {res }}=1100 \mathrm{~K}$ 